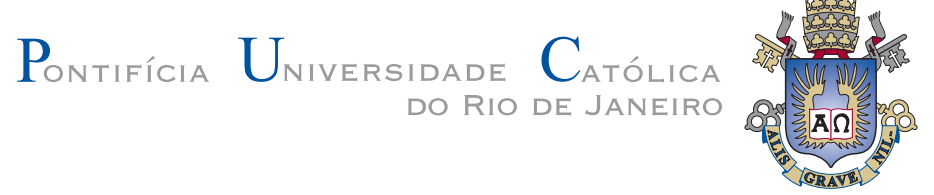

Paulo Roberto Pereira de Souza Filho

\title{
Auxílio a Portabilidade de Código em Aplicações de Alto Desempenho
}

Dissertação apresentada como requisito parcial para a obtenção do grau de Mestre pelo Programa de Pós-graduação em Informática do Departamento de Informática do Centro Técnico e Científico da PUC-Rio.

Orientador: Profa . Noemi de La Rocque Rodriguez 


\title{
Paulo Roberto Pereira de Souza Filho
}

\section{Auxílio a Portabilidade de Código em Aplicações de Alto Desempenho}

Dissertação apresentada como requisito parcial para a obtenção do grau de Mestre pelo Programa de Pós-graduação em Informática do Departamento de Informática do Centro Técnico e Científico da PUC-Rio. Aprovada pela Comissão Examinadora abaixo assinada.

\author{
Prof ${ }^{a}$. Noemi de La Rocque Rodriguez \\ Orientador \\ Departamento de Informática — PUC-Rio
}

Prof. Jairo Panetta

ITA

Prof. Waldemar Celes Filho

Departamento de Informática - PUC-Rio

Prof. Roberto lerusalimschy

Departamento de Informática — PUC-Rio

Prof. Marcio da Silveira Carvalho

Coordenador Setorial do Centro Técnico Científico - PUC-Rio 
Todos os direitos reservados. É proibida a reprodução total ou parcial do trabalho sem autorização da universidade, do autor e do orientador.

\section{Paulo Roberto Pereira de Souza Filho}

Graduou-se em Engenharia da Computação pela Pontifícia Universidade Católica do Paraná (PUC-PR). Trabalha há mais de 13 anos na Petrobras com aplicações de alto desempenho.

Ficha Catalográfica

Souza Filho, Paulo Roberto Pereira de

Auxílio a portabilidade de código em aplicações de alto desempenho / Paulo Roberto Pereira de Souza Filho; orientador: Noemi de La Rocque Rodriguez. - 2016.

117 f. : il. (color.); $30 \mathrm{~cm}$

Dissertação (mestrado) - Pontifícia Universidade Católica do Rio de Janeiro, Departamento de Informática, 2016.

Inclui bibliografia.

1. Informática - Teses. 2. Computação de alto desempenho. 3. Arquiteturas heterogêneas. 4. Vetorização explícita. I. Rodriguez, Noemi de La Rocque. II. Pontifícia Universidade Católica do Rio de Janeiro. Departamento de Informática. III. Título. 


\section{Agradecimentos}

Aos meus Professores Noemi Rodriguez e Jairo Panetta pelo apoio e incentivo para a realização deste trabalho.

À Petrobras e e à PUC-Rio, pelos auxílios concedidos, sem os quais este trabalho não poderia ter sido realizado.

À minha esposa, que me acompanhou todo esse tempo com apoio direto e indireto.

Aos meus pais, família e amigos, que me apoiaram mesmo com minha ausência na vida familiar.

A todos os colegas, professores e funcionários do Departamento de Informática da PUC-Rio, pelo companheirismo, aprendizado e auxílio. 


\section{Resumo}

Souza Filho, Paulo Roberto Pereira de; Rodriguez, Noemi de La Rocque. Auxílio a Portabilidade de Código em Aplicações de Alto Desempenho. Rio de Janeiro, 2016. 117p. Dissertação de Mestrado - Departamento de Informática, Pontifícia Universidade Católica do Rio de Janeiro.

Atualmente na computação de alto desempenho existem diversas opções de arquiteturas de diversos fabricantes, algumas sendo heterogêneas como por exemplo CPU+GPU. Este trabalho tem como objetivo implementar maneiras de codificar aplicações de alto desempenho contemplando alguns tipos de arquiteturas, incluindo algumas heterogêneas, garantindo a portabilidade em uma grande porção do código mas mantendo o desempenho e a capacidade de fazer otimizações específicas a cada arquitetura. Implementamos a biblioteca HLIB que gerencia as primitivas de arquiteturas heterogêneas do tipo CPU+GPU, APU e CPU+Phi e que também funciona em arquiteturas homogêneas tradicionais. Implementamos o OpenVec, uma ferramenta para gerar, de forma portável, código vetorial explícito. Contemplando as principais arquiteturas SIMD dos últimos 17 anos, tais como ARM Neon, Intel SSE até AVX-512 e IBM VSX. Demonstramos o uso combinado dessas duas ferramentas com aplicações de alto desempenho, que demandam mais de um petaflop.

\section{Palavras-chave}

Computação de alto desempenho; Arquiteturas heterogêneas; Vetorização explícita. 


\section{Abstract}

Souza Filho, Paulo Roberto Pereira de; Rodriguez, Noemi de La Rocque (Advisor). Support for Code Portability in High Performance Computing Applications. Rio de Janeiro, 2016. 117p. MSc. Dissertation - Departamento de Informática, Pontifícia Universidade Católica do Rio de Janeiro.

Today's platforms are becoming increasingly heterogeneous. A given platform may have many different computing elements in it: CPUs, coprocessors and GPUs of various kinds. This work propose a way too keep some portion of code portable without compromising the performance along different heterogeneous platforms. We implemented the HLIB library that handles the preparation code needed by heterogeneous computing, also this library transparently supports the traditional homogeneous platform. To address multiple SIMD architectures we implemented the OpenVec, a tool to help compiler to enable SIMD instructions. This tool provides a set of portable SIMD intrinsics and $\mathrm{C}++$ operators to get a portable explicit vectorization, covering SIMD architectures from the last 17 years like ARM Neon, Intel SSE to AVX-512 and IBM Power8 Altivec+VSX. We demonstrated the combination use of this strategy using both tools with petaflop HPC applications.

\section{Keywords}

High performance computing (HPC); Heterogeneous architectures; Explicit vectorization; SIMD; CUDA; OpenCL; hStreams. 


\section{Sumário}

1 Introdução $\quad 12$

$\begin{array}{lll}1.1 & \text { Organização } & 14\end{array}$

2 OpenVec $\quad 15$

2.1 Implementação 20

2.1.1 O Tipo Vetorial 21

2.1.2 Alinhamento e Alocação de Memória 22

2.1.3 Intrínsecos Vetoriais 23

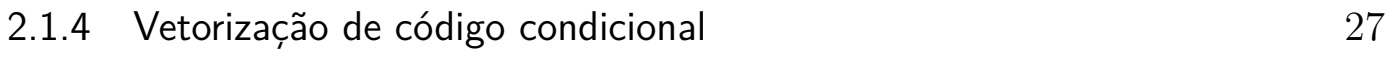

2.1.5 Otimização da vetorização de código condicional 31

2.1.6 Tratamento da cauda dos vetores 33

2.1.7 Reduções 34

$\begin{array}{lll}2.2 & \text { Discussão } & 35\end{array}$

3 Biblioteca de Arquiteturas Heterogêneas $\quad 37$

3.1 Programação de Arquiteturas Heterogêneas 37

3.2 Implementação 43

3.2.1 Inicializar / finalizar dispositivo 44

3.2.2 Alocar / desalocar memória no dispositivo 45

3.2.3 Memória para transferências entre CPU e dispositivo 47

3.2.4 Zerar memória alocada no dispositivo 48

3.2.5 Cópia de memória $\mathrm{CPU} \Leftrightarrow$ dispositivo e dispositivo $\Leftrightarrow$ dispositivo 48

3.2.6 Filas de execução concorrentes $\quad 50$

3.2.7 Passagem de Mensagem entre Dispositivos 54

3.3 Avaliação $\quad 55$

3.4 Discussão $\quad 56$

4 Aplicações de Alto Desempenho $\quad 58$

4.1 Migração Reversa no Tempo RTM $\quad 58$

4.2 Inversão Completa do Campo de Onda FWI 61

4.3 Observações 62

4.4 Exemplo de Uso HLIB 63

4.5 Exemplo de Uso OpenVec $\quad 65$

4.6 Avaliação de Desempenho OpenVec 67

4.7 Uso Combinado HLIB + OpenVec 68

$\begin{array}{lll}4.8 \text { Experimentos } & 69\end{array}$

4.9 Discussão 71

5 Conclusões $\quad 72$

$\begin{array}{lll}6 & \text { Referências Bibliográficas } & \mathbf{7 5}\end{array}$

$\begin{array}{lll}\text { A Documentação OpenVec } & \mathbf{8 4}\end{array}$

A.1 Como Compilar Código OpenVec 84

A.2 Operadores 84 
$\begin{array}{lll}\text { A.3 Constantes } & 85\end{array}$

A.4 Funções $\quad 86$

$\begin{array}{ll}\text { B Documentação HLIB } & 108\end{array}$

$\begin{array}{lll}\text { B.1 Sub-rotinas } & 108\end{array}$

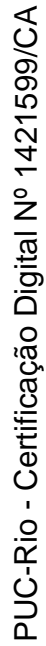




\section{Lista de figuras}

1.1 Aplicação de alto desempenho em três camadas.

2.1 Evolução dos produtos Intel para desktop. A largura SIMD se refere ao número de elementos de precisão simples por operação. Em 1996 existia apenas um núcleo e um float por instrução, em 2016 vários núcleos e 16 floats por instrução. Extraímos os dados da página ark.intel.com. Extrapolamos o clock e o número de núcleos para 2016.

2.2 (a) Adição de vetores escalar. (b) Adição de vetores em uma unidade SIMD de 4 elementos. Definimos o número de iterações do loop com $\mathrm{n}=40$ que é múltiplo da largura da unidade SIMD.

2.3 Formas implícitas e explícitas de vetorização de código.

2.4 Aplicação livre de chamadas proprietárias utilizando o OpenVec, com seus diversos backends.

2.5 Mapeamento dos elementos float no array $\mathrm{x}[\mathrm{]}$ do tipo ov_float, em uma arquitetura com 4 floats por vetor SIMD.

2.6 Operações intermediárias na vetorização de código condicional. Neste exemplo, a condição do if é verdadeira apenas para o segundo e o terceiro elementos.

2.7 Criação da máscara de bits em um processador com uma unidade SIMD com largura OV_FLOAT_WIDTH=4.

3.1 Esquema de conexão em uma arquitetura heterogênea CPU+CPU. Desempenho de conexão baseado na seguinte configuração: CPU Xeon E5-4669 v3, GPU Nvidia K80 conectada via PCle 16X de $3^{a}$ geração.

3.2 Comparativo da evolução do poder computacional em GFlops/s entre Nvidia e Intel. Computação heterogênea em verde, computação homogênea em azul.

3.3 Comparativo entre o grau de portabilidade e desempenho potencial. OpenCL otimizado se refere a múltiplos kernels OpenCL otimizados para cada arquitetura. O desempenho é potencial, fica a cargo do desenvolvedor.

3.4 Aplicação em azul, biblioteca heterogênea em vermelho e as bibliotecas runtime de cada arquitetura em verde.

3.5 A HLIB implementa uma interseção de funcionalidades de diversas APIs.

3.6 Uso do dispositivo ao longo do tempo. Execuções de kernel em azul, cópias de memória em verde e nada sendo executado em vermelho. (a) um exemplo de execução onde o tempo de cópia é menor que o tempo do kernel. (b) um exemplo de execução onde o tempo de cópia é igual ao tempo do kernel.

3.7 Uso das unidades de execução do dispositivo ao longo do tempo. Execuções de kernel em azul, cópias de memória em verde e nada sendo executado em vermelho. 
3.8 Comportamento de dois HLIB_MPI_sendRecv para trocar as bordas com dois processos MPI vizinhos. Eixo do tempo na horizontal. Execuções de kernel em azul, cópias de memória em verde, comunicação MPI em amarelo e nada sendo executado em vermelho. Mensagem quebrada em 6 pedaços. $e_{1}=$ pedaço $o_{1}$ da esquerda do processo corrente, $e v_{1}=$ pedaço $o_{1}$ do vizinho da esquerda, $d_{1}=$ pedaço $o_{1}$ da direita do processo corrente, $v d_{1}=$ pedaço $o_{1}$ do vizinho da direita. $\quad 55$

4.1 Levantamento sísmico marítimo. O navio puxa cabos com os sensores (hidrofones). Abaixo do casco do navio temos a fonte sísmica que é um canhão de ar comprimido. Parte da energia que a fonte produz é refletida pelas camadas geológicas do fundo do mar. 59

4.2 Estêncil para calcular derivadas espaciais de $16^{\mathrm{a}}$ ordem. O cálculo da derivada no ponto central (em vermelho) depende dos pontos vizinhos (em azul).

4.3 Fluxograma do método FWI.

4.4 Troca de bordas do algoritmo de propagação de onda 3D por diferenças finitas, que é utilizado pelas aplicações RTM e FWI. Em verde claro os pontos das bordas necessários aos vizinhos do processo $_{i}$. Os pontos do centro que não são dependência para nenhum outro processo, em verde escuro. O processo $_{i}$ invoca HLIB_MPI_sendRecv duas vezes, conforme as setas.

4.5 (a) um estêncil de 25 pontos $(\mathrm{RAIO}=4)$, obtemos o ponto central em vermelho com uma soma ponderada de todos os pontos. (b) 4 estêncis computados simultaneamente em uma arquitetura SIMD com 4 elementos por vetor.

4.6 Cálculo simultâneo de 16 elementos em uma arquitetura SIMD com 16 elementos por vetor, como o Intel Xeon Phi.

4.7 Desempenho do estêncil de $16^{\mathrm{a}}$ ordem com OpenVec+OpenMP e com o código de referência, que utiliza intrínsecos AVX-2 e OpenMP. Ambiente do teste: processador Xeon E5-2697v3 @ 2.60 $\mathrm{GHz}$ com 14 núcleos, compilador da Intel versão 15.0.1.133 e gcc 4.4.7.

4.8 Camadas da RTM com HLIB+OpenVec. A RTM utiliza a HLIB para fazer o gerenciamento da computação heterogênea. Codificamos os kernels das arquiteturas hStreams e regular com o OpenVec. 68

4.9 Camada de arquitetura da RTM. As setas indicam a direção de invocação. Por exemplo, o driver do código regular invoca o kernel OpenVec. 


\section{Lista de tabelas}

2.1 Largura das unidades SIMD em cada arquitetura suportada pelo OpenVec.

2.2 Intrínsecos OpenVec para precisão simples.

2.3 Intrínsecos de comparação para precisão simples e operadores relacionais (apenas $\mathrm{C}++$ ). Esses intrínsecos retornam uma máscara de bits com os bits ligados nos elementos onde a comparação é verdadeira.

2.4 Intrínsecos de redução para máscaras de comparação entre objetos do tipo ov_float. Esses intrínsecos também estão disponíveis para precisão dupla (sufixo "d").

2.5 Intrínsecos de redução para o testar se os elementos de um objeto ov_float são negativos. Esses intrínsecos também estão disponíveis para precisão dupla (sufixo "d").

2.6 Funções de redução matemática, com $n=$ OV_FLOAT_WIDTH-1. O Open Vec implementa as mesmas funções para precisão dupla com o sufixo $d$.

3.1 Primitivas HLIB da computação heterogênea.

3.2 Inicialização interna da HLIB em diferentes arquiteturas.

4.1 Estimativa da quantidade de operações de ponto flutuante para uma migração RTM. O total de operações é o produto das demais linhas da tabela. As dimensões comprimento, largura e profundidade compreendem um volume discretizado em uma grade regular 3D.

4.2 Experimentos com diversos dispositivos e APIs. A primeira coluna se refere ao número do experimento, e a última ao ano de lançamento do dispositivo.

A.1 Flags de compilação para diferentes compiladores e arquiteturas. 


\section{Introdução}

As aplicações de alto desempenho podem comprometer orçamentos de dezenas de milhões de reais entre aquisições de supercomputadores e gastos operacionais com CPDs. O maior supercomputador divulgado, o Tianhe-2 com capacidade de 33.86 petaflops[1], tem um custo estimado de US\$ 390 milhões e consome aproximadamente US\$ 24 milhões por ano com energia elétrica ${ }^{1}$.

Os desenvolvedores das aplicações que executam neste ambiente de super computação têm a difícil decisão de escolher o nível de otimização de código a ser usado, pois um ganho de desempenho, mesmo que pequeno, pode trazer uma grande economia de recursos. Em alguns casos, essa decisão envolve a escolha da linguagem de programação antes do início do projeto. Muitas opções de otimização são limitadas a um determinado fabricante ou arquitetura e normalmente não são portáveis a outros tipos e modelos de supercomputadores.

Nos últimos anos, as arquiteturas heterogêneas, que utilizam a CPU em conjunto com um acelerador, ganharam espaço na lista dos 500 maiores supercomputadores do mundo[3], sendo que aparecem nos dois primeiros lugares nessa lista. Esse tipo de arquitetura torna ainda mais difícil a decisão do desenvolvedor de definir o nível de otimização a ser aplicado em um determinado código.

Determinados níveis de otimização deixam o código menos legível e dependente de um determinado fornecedor de hardware. Com isso fica mais difícil adicionar novas características e mover o código para outras arquiteturas e outros fornecedores. Além disso, no caso de arquiteturas heterogêneas, existe uma complexidade adicional de manter dois códigos: um para arquitetura homogênea tradicional e outro para a arquitetura heterogênea. Por exemplo, ao escolher codificar em Fortran ou C, o desenvolvedor garante que seu código irá abranger a maior parte dos supercomputadores. Mas o desempenho em uma arquitetura heterogênea com GPUs Nvidia fica muito distante do desempenho potencial de se codificar diretamente em CUDA[4]. Por outro lado, ao utilizar CUDA o desenvolvedor limita seu código a apenas um tipo de arquitetura.

Acreditamos que a estratégia de dividir a aplicação em camadas ajuda a manter uma parte do código portável, por exemplo. Propomos dividir a aplicação de alto desempenho heterogênea em três camadas: uma camada superior agnóstica de arquitetura, uma camada com as primitivas de gerenciamento da computação heterogênea e outra camada com o código que efetivamente executa no dispositivo secundário, que em geral é a porção de código da aplicação

\footnotetext{
${ }^{1}$ Custos de energia elétrica baseados no mercado dos EUA[2].
} 
que se deseja acelerar com o uso de um dispositivo secundário, conforme a Figura 1.1.

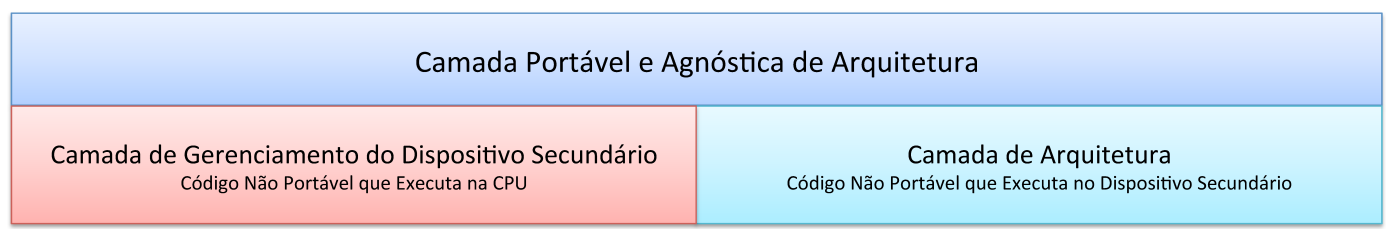

Figura 1.1: Aplicação de alto desempenho em três camadas.

O objetivo deste trabalho é prover ferramentas para o auxílio à portabilidade de código em aplicações de alto desempenho com o foco no desempenho, e propor uma estratégia para organizar essas aplicações em três camadas bem distintas. Uma das nossas ferramentas implementa toda a camada de gerenciamento (em vermelho) da Figura 1.1. Ao dividir a aplicação em três camadas o desenvolvedor isola suas otimizações específicas de cada arquitetura na camada de arquitetura, onde cada arquitetura fica isolada em arquivos fonte distintos. Nossa ferramenta implementa a camada de gerenciamento, e o resto do código da aplicação fica isolado em uma outra camada portável e agnóstica de arquitetura.

Implementamos duas ferramentas que trazem um balanceamento entre portabilidade e desempenho.

A primeira ferramenta explora o uso das unidades vetoriais dos processadores, como a unidade do processador Xeon Phi do supercomputador Tianhe-2. Essa ferramenta habilita o desenvolvedor a expor seu código de uma forma que o compilador produza instruções vetoriais.

As unidades vetoriais são capazes de processar vários elementos simultaneamente em paralelo com apenas uma instrução. Esse tipo de unidade é chamada SIMD (single instruction multiple data) e é utilizada por CPUs, APUs e GPUs. A maioria dos supercomputadores atuais possui unidades SIMD.

O modo de expor paralelismo do tipo SPMD (single program multiple data) entre diferentes nós e entre diferentes núcleos está bem definido, com padrões abertos como o MPI e o OpenMP. Mas atualmente não existe um padrão aberto para expressar um paralelismo do tipo SIMD de forma explícita. Nossa ferramenta provê funções vetoriais SIMD portáveis que são mapeadas diretamente para funções SIMD específicas de cada arquitetura. Ao utilizar nossa ferramenta de vetorização explícita, o desenvolvedor não se refere a característica alguma da unidade SIMD. Com isso, o mesmo código pode ser compilado para qualquer largura SIMD, inclusive para uma arquitetura escalar com apenas um elemento por instrução. 
A segunda ferramenta que implementamos auxilia o desenvolvedor na programação de arquiteturas heterogêneas como CPU+GPU. Na computação heterogênea o desenvolvedor pode gastar muito tempo em um código que prepara o dispositivo para execução. Esse código de preparação é o responsável por tarefas como inicializar o dispositivo, alocar e povoar sua memória e enfileirar tarefas a serem executadas no dispositivo. Nossa ferramenta é uma biblioteca com primitivas básicas da computação heterogênea. Essas primitivas implementam de forma portável a porção de código que faz a preparação dos dispositivos secundários. Ao utilizar nossa biblioteca o desenvolvedor pode focar no desenvolvimento do código que efetivamente executa no dispositivo. Essa ferramenta trata a arquitetura homogênea como uma arquitetura heterogênea, permitindo que o desenvolvedor mantenha apenas uma lógica de gerenciamento que contempla arquiteturas homogêneas e heterogêneas.

Como o desenvolvedor somente utiliza chamadas específicas/proprietárias no código que executa no dispositivo, esse código fica naturalmente isolado em uma camada de software, que chamamos de camada de arquitetura. Com isso, o resto do código da aplicação fica neutro em relação à arquitetura, isolado em outra camada de software que invoca a nossa biblioteca.

Para demonstrar o uso desta estratégia com as duas ferramentas combinadas, discutimos a implementação da aplicação de migração reversa no tempo[5]. As equipes de processamento sísmico utilizam essa aplicação para gerar uma imagem de subsuperfície que auxilia a tomada de decisão do posicionamento de um poço de petróleo, que pode custar US\$ 50 milhões. Em áreas geológicas complexas como o pré-sal, essa migração posiciona os eventos geológicos de forma mais correta que outras migrações que demandam menos poder computacional. Uma única execução da migração reversa no tempo pode ocupar uma máquina de um petaflop por três meses.

\section{1}

\section{Organização}

Esta dissertação está organizada da seguinte forma. Nos Capítulos 2 e 3 apresentamos nossas duas ferramentas de auxílio à portabilidade de código em aplicações de alto desempenho. No Capítulo 4 apresentamos aplicações de alto desempenho que demandam grandes recursos computacionais, e discutimos o uso combinado das nossas ferramentas nessas aplicações. Em seguida, concluímos esta dissertação no Capítulo 5. No final do texto, nos Anexos A e B disponibilizamos a documentação das duas ferramentas que implementamos. 


\section{2 \\ OpenVec}

Há mais de uma década os fabricantes de processadores não podem mais aumentar o desempenho dos processadores simplesmente aumentando a frequência: o power wall[6], um limite de potência por área, inviabilizou o aumento da frequência de operação dos processadores. O aumento indiscriminado da frequência levaria a temperaturas insustentáveis. Desde 2003 os processadores não dependem do aumento da frequência de operação como fonte primária de aumento de desempenho. Eles ficam mais largos a cada nova geração, podendo executar mais operações simultaneamente.

Esse aumento na largura do processador pode ocorrer de várias formas, sendo que as principais são o aumento do número de núcleos, como por exemplo um processador quad-core, unidades vetoriais SIMD (single instruction multiple data), como a unidade Neon do processador ARM que podemos encontrar em smartphones, e o uso de múltiplas unidades de execução em paralelo como load, store, integer e SIMD.

Do ponto de vista do programador, duas tendências ficam claras[7]: aplicações multi-thread e paralelismo ao nível de instrução ILP (instruction level paralelism) com instruções SIMD. Existem várias formas populares e bem estabelecidas de explorar os diversos núcleos de processamento, incluindo padrões abertos, tais como MPI, OpenMP, TBB, pThreads e OpenCL. Entretanto não existe nenhum padrão maduro ou amplamente utilizado para explorar a vetorização de maneira explícita. Recentemente o padrão OpenMP 4.0 trouxe algumas características dedicadas a vetorização. Apesar de alguns compiladores fazerem um bom trabalho de vetorização automática e da notação de array presente em algumas linguagens como Fortran 90, bem como das extensões de anotação de código presente em alguns compiladores, não é possível para o programador mapear diretamente instruções vetoriais de forma portável. A notação de array, apesar de portável e padrão, fornece apenas uma dica ao compilador de que aquelas operações podem ser vetorizadas.

Apesar do modelo de programação de múltiplos núcleos estar muito mais maduro e padronizado, nos últimos quinze anos o aumento no desempenho dos sistemas de computação se deu, tanto pelo uso de unidades SIMD, como pelo aumento do número de núcleos. A Figura 2.1 mostra a evolução dos processadores Intel para desktop com relação ao clock, número de núcleos e a largura da unidade vetorial SIMD (capacidade de processar mais de um elemento por instrução).

As arquiteturas como as GPUs, que privilegiam unidades SIMD em detrimento das outras unidades, têm um desempenho por Watt maior em 
relação às CPUs tradicionais, que gastam mais espaço de silício com caches e execução fora de ordem.

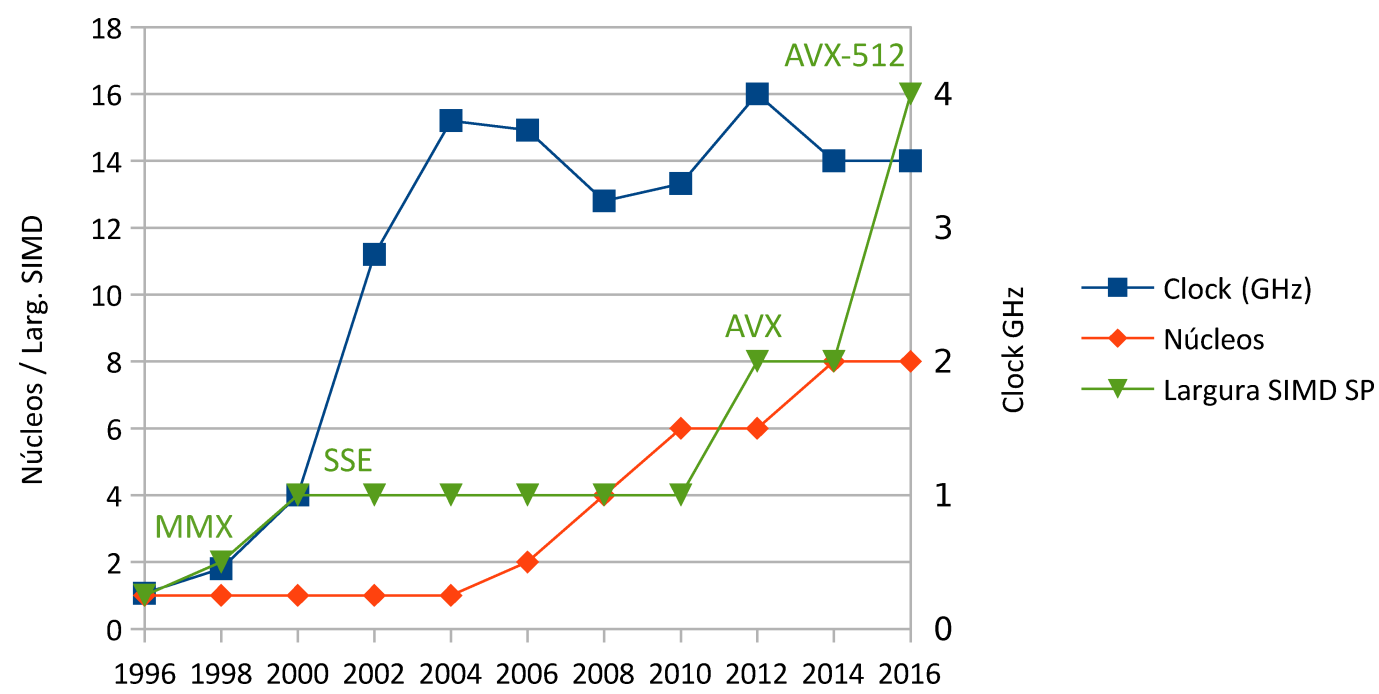

Figura 2.1: Evolução dos produtos Intel para desktop. A largura SIMD se refere ao número de elementos de precisão simples por operação. Em 1996 existia apenas um núcleo e um float por instrução, em 2016 vários núcleos e 16 floats por instrução. Extraímos os dados da página ark.intel.com. Extrapolamos o clock e o número de núcleos para 2016.

A Intel lançou as tecnologias MMX e SSE antes de lançar seu processador dual-core. A unidade SIMD MMX processa dois floats simultaneamente e a SSE quatro floats. O processador Intel Haswell, lançado em 2013, dobrou o número de unidades SIMD por núcleo em relação à geração anterior, sendo capaz de processar 2x8 FMAs (fused multiply and add) simultaneamente por núcleo. A Intel anunciou para 2016 o lançamento da unidade vetorial AVX-512 com 16 elementos por instrução.

Como o power wall limita o aumento de frequência de operação dos processadores, imaginamos, que cada vez mais, o aumento de desempenho venha da utilização de instruções vetoriais. Por exemplo, um processador Intel Core i7-5960X é capaz de processar 128 (8x2x8) floats simultaneamente com seus 8 núcleos, cada um com duas unidades vetoriais de 8 elementos (AVX 2). Atualmente a maioria dos processadores possui unidades SIMD, incluindo os processadores de smartphones e tablets.

Para extrair todo o potencial desse tipo de processador, é necessário que o compilador gere instruções vetoriais[8]. Embora se tenha avançado nas técnicas de vetorização automática $[9,10,11,12,13,14,15,16,17]$, os compiladores ainda não vetorizam alguns códigos[18, 19, 9, 8, 20]. Uma das opções para solucionar esse problema é o uso de SIMD intrinsics[21, 22, 23, 24], que são funções fornecidas pelo compilador que são mapeadas diretamente em uma 
ou mais instruções SIMD do processador. Neste trabalho utilizaremos o termo intrínsecos para descrever SIMD intrinsics.

Diversos trabalhos reportam ganhos de desempenho com a utilização de intrínsecos $[25,26,27,28,29,30,31,32,33]$. O grande problema dessa abordagem é que esses intrínsecos são específicos para cada arquitetura. Um código que utiliza intrínsecos Intel SSE, por exemplo, não extrai todo o potencial de um processador Intel AVX, apesar de ambos serem desenvolvidos pelo mesmo fabricante, e um código AVX não executa em um processador SSE. Com isso, uma recodificação é necessária para cada arquitetura.

Para exemplificar, vamos discutir o caso de uma adição simples de vetores, como a mostrada na Figura 2.2a. Podemos mapear a adição de vetores da Figura 2.2a para uma instrução SIMD que soma vários elementos simultaneamente em paralelo, conforme a Figura 2.2b.

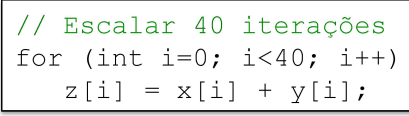

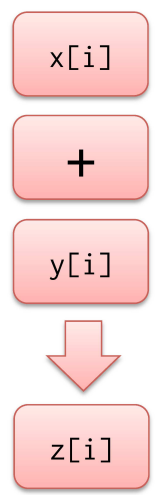

(a)
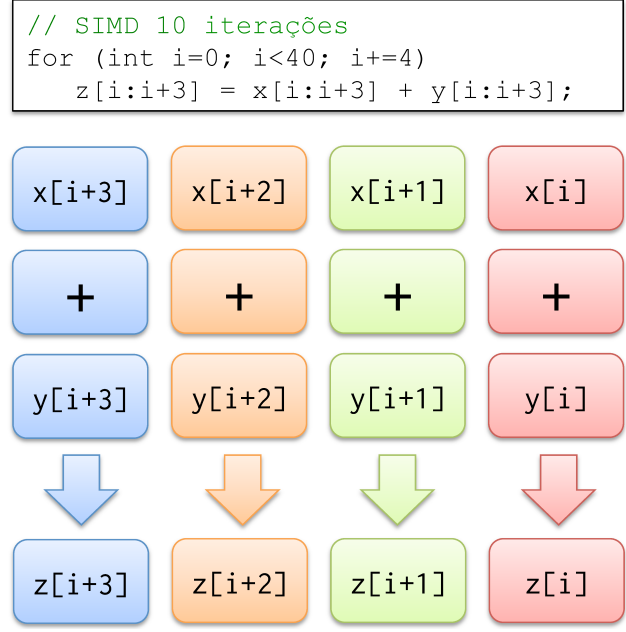

(b)

Figura 2.2: (a) Adição de vetores escalar. (b) Adição de vetores em uma unidade SIMD de 4 elementos. Definimos o número de iterações do loop com $\mathrm{n}=40$ que é múltiplo da largura da unidade SIMD.

Atualmente a unidade SIMD com mais elementos pertence ao processador Xeon Phi, com 16 elementos por instrução. Essa largura de 16 operandos por instrução habilita um ganho teórico de desempenho de 16 vezes, tendo em vista que cada instrução processa 16 elementos de cada vez. Na prática é difícil obter $100 \%$ do ganho teórico pois a velocidade da memória pode não ser capaz de alimentar todos esses operandos simultâneos. De forma simplificada, a relação do desempenho atingido em relação ao pico teórico fica limitada pela intensidade computacional da aplicação. Williams[31] define a intensidade computacional como a razão entre a quantidade de operações de ponto flutuante e a quantidade de acessos à memória, utilizando a unidade $\frac{\text { flops }}{\text { word }}$. 
Para lidar com a falta de portabilidade dos intrínsecos proprietários, desenvolvemos o OpenVec[34, 35], que apresentamos a seguir. O OpenVec é uma ferramenta para geração explícita de código vetorial SIMD para ser aplicada nos hot spots de uma aplicação, com o objetivo de obter um desempenho similar ao codificado com intrínsecos proprietários mas mantendo a aparência e a portabilidade de um código de alto nível.

A Figura 2.3 mostra as diversas formas de vetorizar um código. Algumas formas são implícitas, como por exemplo o uso de diretivas (\#pragma) para guiar o compilador na vetorização de um loop.

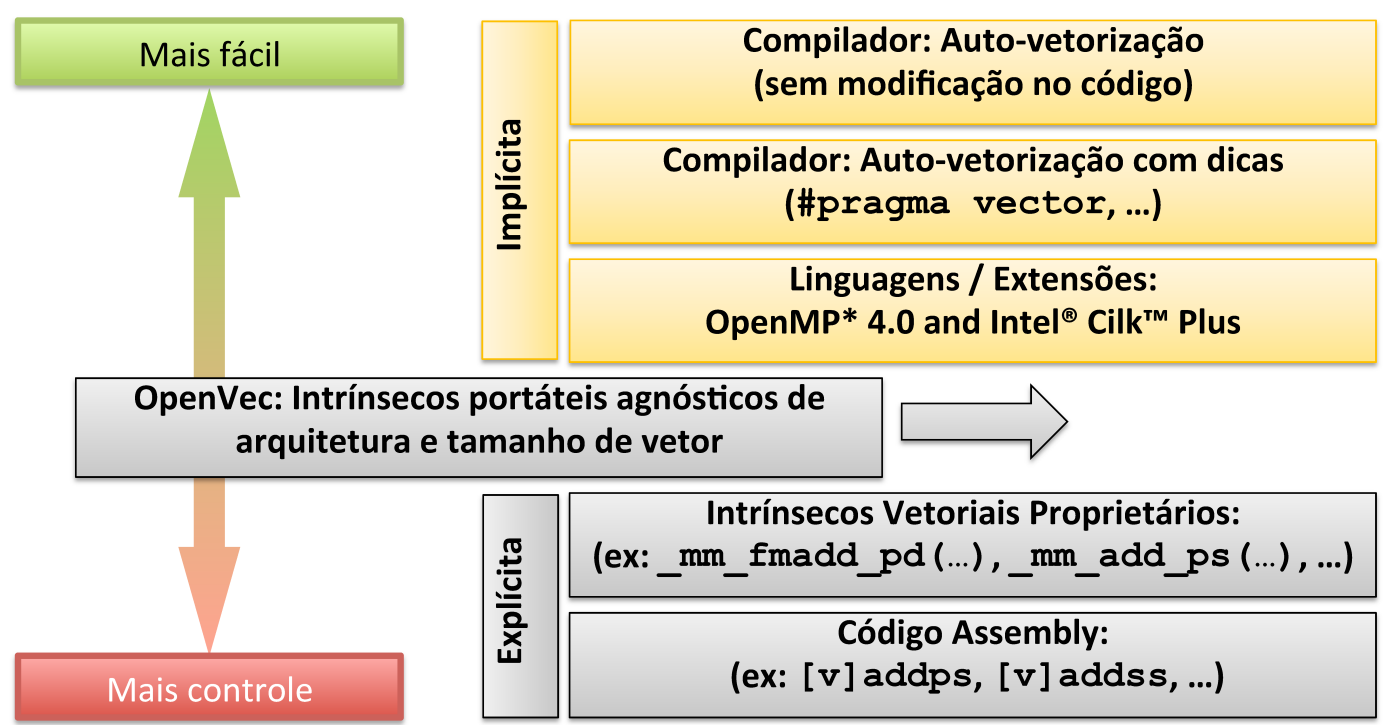

Figura 2.3: Formas implícitas e explícitas de vetorização de código.

O OpenVec define um tipo vetorial e o suporte aos intrínsecos de hardware para as linguagens $\mathrm{C}$ e $\mathrm{C}++$, através de macros e sobreposição de operadores (operator overload) no caso de $\mathrm{C}++$. Ele mapeia as APIs proprietárias de cada arquitetura/compilador para uma API comum e portável.

Também geramos código escalar para os casos onde a arquitetura não possui instruções SIMD ou para uma arquitetura não suportada. Com isso garantimos que um código OpenVec seja compilado em qualquer arquitetura por qualquer compilador padrão $\mathrm{C} / \mathrm{C}++$. O backend escalar do OpenVec é o responsável por gerar esse código.

O OpenVec tem como público alvo desenvolvedores que desejam utilizar intrínsecos SIMD para acelerar hot spots em diversos tipos e gerações de processadores mantendo o reuso e a portabilidade de código. Testamos nossa implementação em diversos processadores - ARM Neon, Intel SSE/AVX/AVX512/IMCI e IBM Power8 VSX - e com diversos compiladores - gcc, llvm e Intel. Nossa implementação utiliza apenas header files com o preprocessador 
C e no caso de $\mathrm{C}++$ também utilizamos sobreposição de operadores. Também testamos o backend escalar que funciona com qualquer compilador.

A Figura 2.4 mostra a aplicação em uma camada livre de chamadas proprietárias e os diversos backends do OpenVec. Organizamos a implementação de cada arquitetura (backend) em header files separados. O header file principal openvec.h define o backend em tempo de compilação. O desenvolvedor usuário do OpenVec inclui em seu código apenas o header file principal.

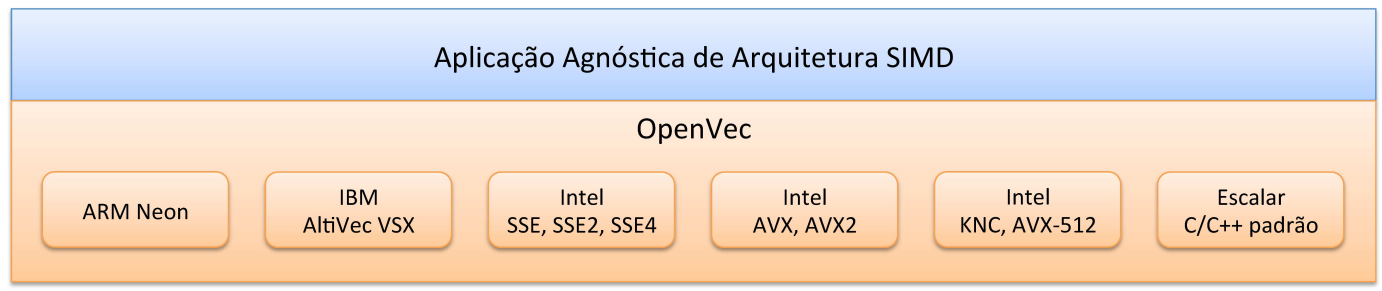

Figura 2.4: Aplicação livre de chamadas proprietárias utilizando o OpenVec, com seus diversos backends.

A Listagem 2.1 mostra a função SAXPY da BLAS, implementada de forma escalar e com o OpenVec. Essa função multiplica elemento a elemento um vetor por um escalar, e soma o resultado com um outro vetor.

Listagem 2.1: SAXPY escalar e com OpenVec C++ e C.

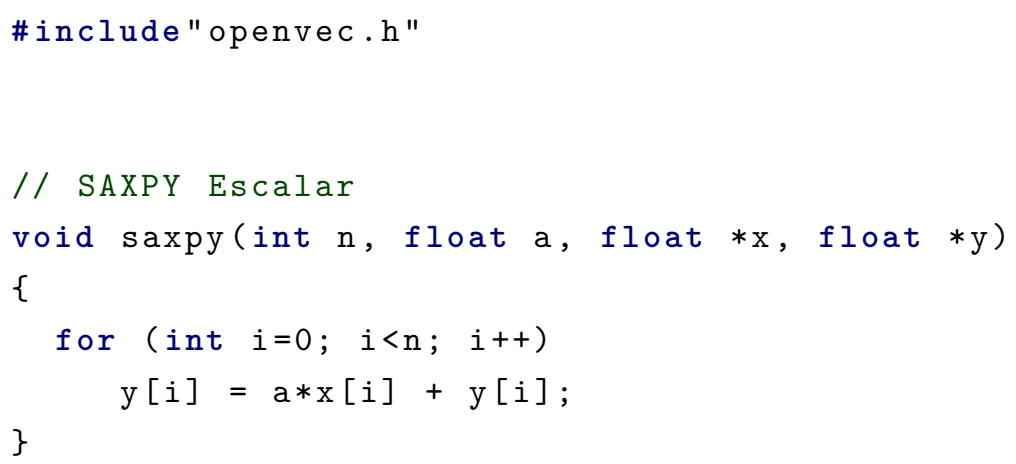




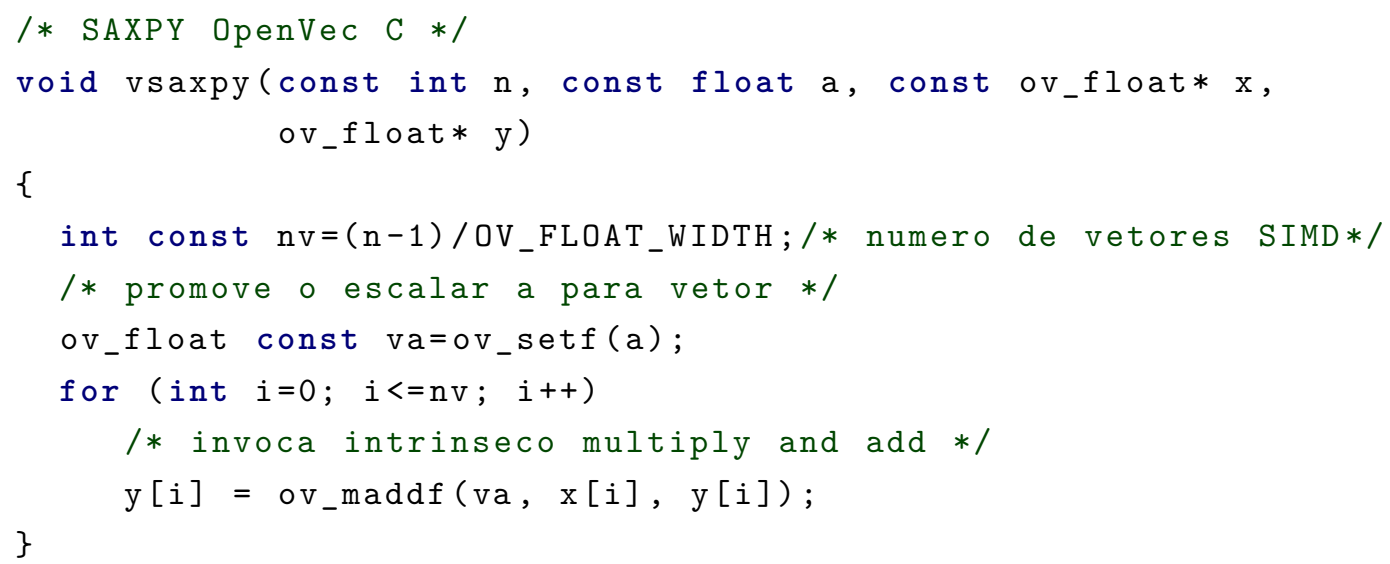

Na Listagem 2.1, os arrays x [ ] e y [ ] são declarados com o tipo vetorial ov_float que contém, por exemplo, 4 floats (128 bits) em um processador IBM Power 8. Cada backend implementa a constante OV_FLOAT_WIDTH, que define a largura da unidade SIMD para floats (precisão simples).

A Figura 2.5 mostra o mapeamento dos vinte primeiros elementos do array de floats da Listagem 2.1 em um array de vetores SIMD do tipo ov_float.

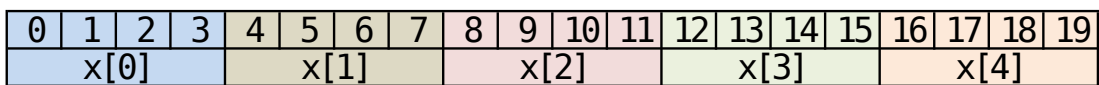

Figura 2.5: Mapeamento dos elementos float no array x [ ] do tipo ov_float, em uma arquitetura com 4 floats por vetor SIMD.

O header file openvec.h. define a operação a*x[i] (multiplicação de escalar por vetor) da Listagem 2.1 com um overload de operador $\mathrm{C}++$.

Podemos observar na Listagem 2.1 que a versão do código que utiliza C é mais complexa que a versão $\mathrm{C}++$. Em $\mathrm{C}$, precisamos promover o escalar para vetor e invocar um intrínseco para efetuar a multiplicação e soma.

A Seção 2.1.6 trata o caso onde o número de elementos do loop (variável n da Listagem 2.1) não é múltiplo da largura do vetor SIMD.

\section{1}

\section{Implementação}

Implementamos o OpenVec apenas com macros padrão do preprocessador C, garantindo com isso o mínimo de overhead pois os intrínsecos OpenVec são substituídos por intrínsecos proprietários do compilador pelo preprocessador C. Em $\mathrm{C}++$ também utilizamos a sobreposição de operadores. O OpenVec não é uma biblioteca, e sim um header file que mapeia os intrínsecos proprietários do compilador para uma nomenclatura comum e agnóstica de arquitetura.

Criamos backends para as arquiteturas SIMD a seguir: 
- ARM Neon

- Intel SSE/SSE2/SSE4/AVX/AVX-2/AVX-512/IMCI

- IBM Power8 VSX

- Escalar, compatível com qualquer compilador $\mathrm{C} / \mathrm{C}++$

\subsection{1}

\section{O Tipo Vetorial}

O OpenVec define tipos vetoriais que o compilador mapeia para registradores vetoriais. Esses tipos contém múltiplos elementos e seu tamanho varia conforme a largura SIMD de cada arquitetura. Definimos a constante OV_FLOAT_WIDTH para cada backend com o número de valores de ponto flutuante da unidade SIMD (precisão simples) do processador. No backend escalar OV_FLOAT_WIDTH=1.

O tipo ov_float define uma variável que contém OV_FLOAT_WIDTH valores de ponto flutuante em precisão simples (floats). Por exemplo, em um processador ARM Neon o tipo ov_float contém 4 floats, em um processador Intel AVX ov_float contém 8 floats. Utilizamos esse tipo para declarar variáveis da mesma forma que os tipos nativos, como float e double. A seguir um exemplo de declaração de uma variável vetorial de floats:

ov_float minhaVar;

Cada arquitetura define um tipo vetorial diferente, e cada uma possui uma largura SIMD. Por exemplo, em um processador ARM Neon mapeamos o tipo ov_float para o tipo proprietário float32x4_t que contém 4 floats, enquanto em um processador Intel AVX mapeamos o mesmo ov_float para o tipo __m256 que contém 8 floats.

Apos instanciar uma variável com o tipo vetorial, todas as operações serão executadas simultaneamente em todos os elementos da variável. Por exemplo, em um processador Intel SSE com OV_FLOAT_WIDTH=4, a soma $x+y$ com duas variáveis do tipo ov_float, executa em paralelo, somando cada float de $x$ com o respectivo float de $y$.

Definimos as operações entre variáveis escalares e vetoriais repetindo o valor escalar para todas as posições do vetor. Por exemplo, a soma $x+1$, com $x$ sendo do tipo ov_float, executa em paralelo da seguinte forma em um processador Intel SSE:

$$
x_{0}+1 ; x_{1}+1 ; x_{2}+1 ; x_{3}+1 ;
$$

Implementamos operações vetoriais com números de ponto flutuante de 32 e 64 bits, com os tipos ov_float e ov_double e as respectivas constantes OV_FLOAT_WIDTH e OV_DOUBLE_WIDTH. 
A Tabela 2.1 mostra a largura SIMD para todas as arquiteturas suportadas pelo OpenVec.

\begin{tabular}{|l|c|c|}
\hline Arquitetura SIMD & OV_FLOAT_WIDTH & OV_DOUBLE_WIDTH \\
\hline Intel Xeon Phi AVX-512 & 16 & 8 \\
\hline Intel AVX/AVX-2 & 8 & 4 \\
\hline Intel SSE2/SSE4 & 4 & 2 \\
\hline Intel SSE & 4 & 1 \\
\hline ARM Neon & 4 & 1 \\
\hline IBM Power Altivec & 4 & 1 \\
\hline Escalar & 1 & 1 \\
\hline
\end{tabular}

Tabela 2.1: Largura das unidades SIMD em cada arquitetura suportada pelo OpenVec.

\subsection{2}

\section{Alinhamento e Alocação de Memória}

Em todas as arquiteturas SIMD existe uma penalidade de desempenho se uma estrutura não está alinhada na memória. Para executar um load ou store sem penalidade de desempenho, o endereço base da posição de memória deve estar no alinhamento natural do vetor. Por exemplo, em um processador Xeon Phi, ao carregar uma variável ov_float da memória, a posição de memória deve estar alinhada da seguinte forma:

OV_ALIGN $=$ OV_FLOAT_WIDTH $\mathrm{x}$ sizeof $(\mathrm{float})=16 \mathrm{x} 4=64$ bytes

No OpenVec, definimos a constante OV_ALIGN, usando o cálculo acima, para indicar qual é a restrição de alinhamento de cada arquitetura.

Implementamos as seguintes funções de alocação de memória que garantem que o primeiro elemento alocado está alinhado em OV_ALIGN bytes:

void *ov_malloc(size_t size);

void ov_free (void *ptr);

void *ov_calloc(size_t count, size_t size);

Uma memória alocada com as funções ov_malloc e ov_calloc deve ser desalocada somente com a função ov_free.

Como o desenvolvedor tem a informação de que o primeiro elemento está alinhado, ele pode, em alguns casos, garantir que os demais acessos à memória serão alinhados, por exemplo, fazendo com que a dimensão contígua em memória de seus arrays seja múltipla de OV_FLOAT_WIDTH. Em um array alinhado, o primeiro elemento está sempre alinhado e temos um endereço alinhado a cada OV_FLOAT_WIDTH elementos. 
Tratar o alinhamento de memória traz um ganho de desempenho[36]. Com o OpenVec é possível informar o compilador se o acesso é alinhado ou não. A Seção 2.1.3 detalha este procedimento.

Para auxiliar o desenvolvedor no caso onde o tamanho do array não é múltiplo de OV_FLOAT_WIDTH, as funções de alocação adicionam OV_ALIGN bytes na quantidade de bytes alocados. Dessa forma o desenvolvedor não precisa testar se o tamanho do array é múltiplo de OV_FLOAT_WIDTH, basta deixar o código "invadir" as ultimas posições de memória, como mostra a Listagem 2.1.

Em todos os backends, com exceção do escalar, nossa implementação invoca a função posix_memalign, que aloca memória alinhada. Por conveniência, o header file openvec.h substitui as funções padrão malloc, calloc e free pelas funções equivalentes do OpenVec. Dessa forma, o desenvolvedor não precisa modificar o código para obter o beneficio da alocação alinhada. No backend escalar, para manter o máximo de compatibilidade, nossa implementação invoca as funções padrão malloc, calloc e free.

\subsection{3}

\section{Intrínsecos Vetoriais}

A programação com intrínsecos vetoriais SIMD requer que o desenvolvedor utilize funções (intrínsecos) que na maioria dos casos se referem a uma instrução SIMD do processador. Além disso o desenvolvedor deve substituir o uso dos tipos básicos como float e double por tipos vetoriais que contêm múltiplos elementos. A codificação de algumas operações triviais é diferente na programação com intrínsecos, por exemplo, não é possível utilizar um if-then-else, que deve ser substituído por um intrínseco. Em alguns casos, para acessar a memória é necessário utilizar intrínsecos de load e store.

Implementamos diversas funções que mapeiam os intrínsecos proprietários dos compiladores. As chamadas OpenVec normalmente fazem um mapeamento direto utilizando macros, conforme a definição a seguir do nosso backend Intel AVX:

\#define ov_sqrtf_mm256_sqrt_ps

Em $\mathrm{C}++$ o uso de algumas funções matemáticas básicas é desnecessário, pois existe o overload de operadores, conforme exemplo a seguir:

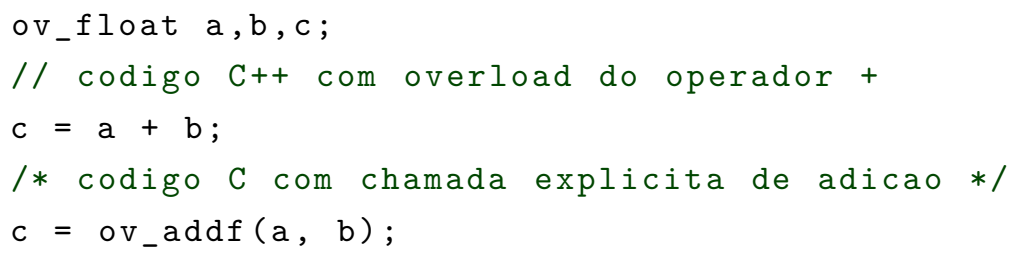


Em um código $\mathrm{C} / \mathrm{C}++$ com os tipos básicos da linguagem, o compilador gera automaticamente as operações load e store. No OpenVec é possível programar com load e store implícitos (gerados pelo compilador) e explícitos.

O desenvolvedor pode declarar seus arrays com um tipo básico como float e utilizar LD/ST explícitos ou mapear os arrays para um tipo vetorial como o ov_float resultando em LD/ST implícitos. Todo acesso implícito deve ser alinhado. Essa restrição também existe para os tipos básicos, por exemplo, um acesso a um float deve estar alinhado na memória em 4 bytes. Se o desenvolvedor faz um acesso implícito a um tipo básico ou vetorial em um endereço desalinhado, o programa termina com o sinal SIGSEGV (segmentation fault). A Listagem 2.2, mostra o uso do LD/ST implícito e explícito:

Listagem 2.2: Duas implementações de SAXPY com LD/ST explícitos e implícitos.

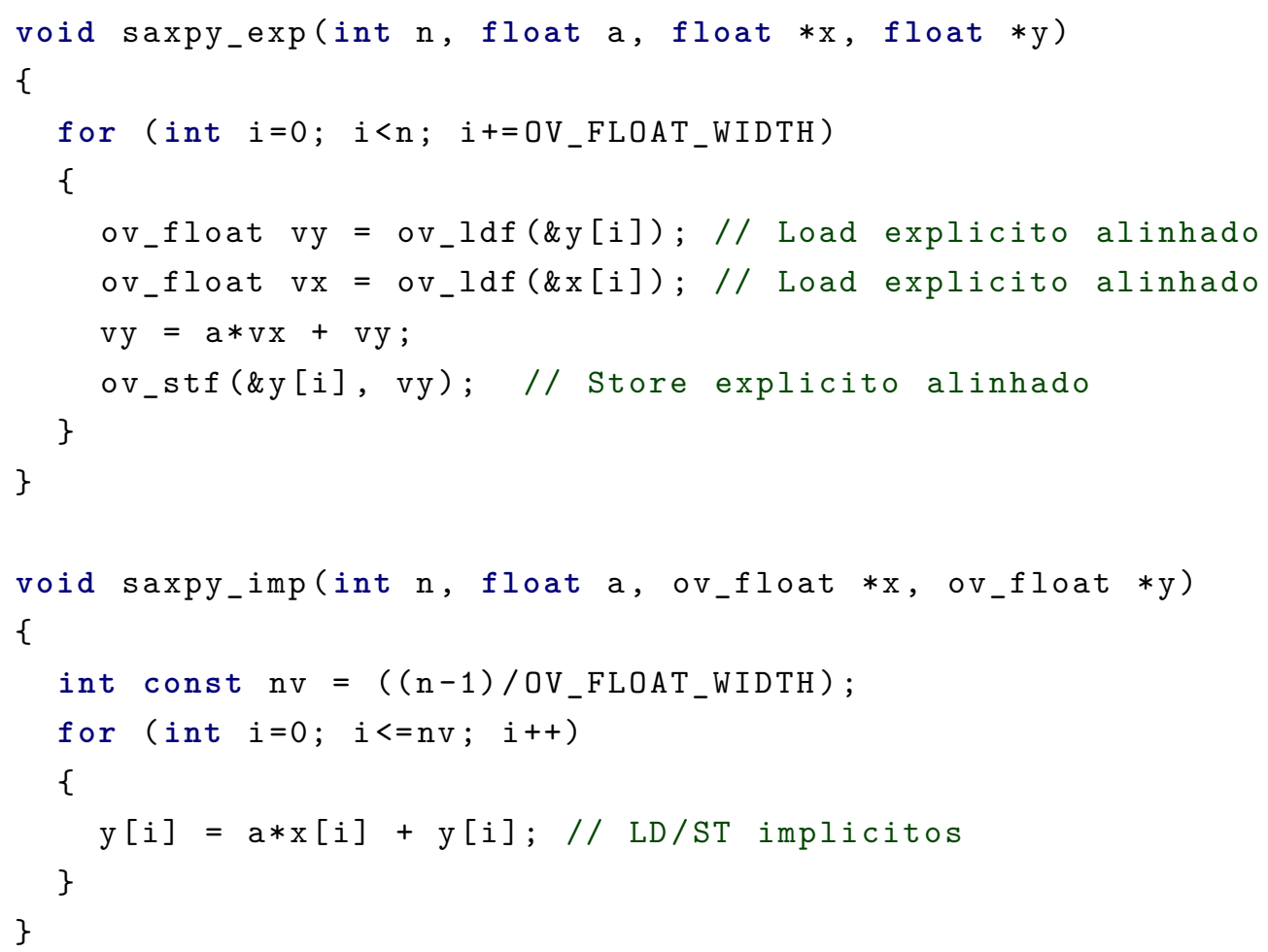

O desempenho dos acessos alinhados é maior que o desempenho dos acessos desalinhados[36]. Mas se o desenvolvedor não pode assumir com segurança que o endereço está alinhado, ele deve utilizar o acesso explícito desalinhado. Se um array do tipo ov_float tem o primeiro elemento alinhado, todos os outros elementos ov_float do array estão alinhados.

Implementamos funções que testam se um endereço está alinhado ou não. Se o desenvolvedor não tem o controle da alocação de memória, ele pode escrever duas versões de código: uma alinhada e outra não. A Listagem 2.3 
apresenta a invocação de duas versões da função SAXPY. Neste exemplo, o desenvolvedor testa o alinhamento de memória dos arrays $\mathrm{x}[]$ e y [].

Listagem 2.3: Teste de alinhamento de memória.

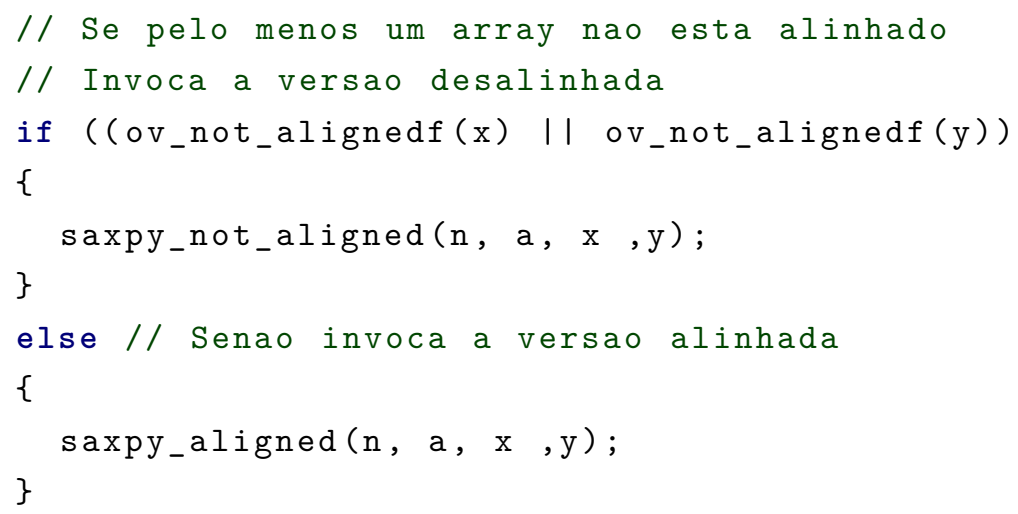

O desenvolvedor deve tomar cuidado com a semântica do acesso implícito. Por exemplo, no array do tipo ov_float a indexação x $[i+1]$ aponta para o próximo vetor e não para o próximo float.

A Tabela 2.2 mostra todas as funções OpenVec para precisão simples, que recebem o sufixo " $f$ ". As funções que operam no tipo ov_double recebem o sufixo "d". 


\begin{tabular}{|c|c|}
\hline Intrínseco SIMD & Operação / Descrição \\
\hline ov_addf $(x, y)$ & $x+y$ \\
\hline ov_subf $(x, y)$ & $x-y$ \\
\hline ov_mulf $(x, y)$ & $x \times y$ \\
\hline ov_divf $(x, y)$ & $\frac{x}{y}$ \\
\hline ov_ldf(endereço) & Load alinhado \\
\hline ov_uldf (endereço) & Load desalinhado \\
\hline ov_stf (endereço, $x$ ) & Store alinhado \\
\hline ov_storeuf (endereço, $\mathrm{x}$ ) & Store desalinhado \\
\hline ov_stream_stf (endereço, x) & Stream store alinhado \\
\hline ov_nt_storef (endereço,x) & Store não temporal alinhado \\
\hline ov_setzerof $(x)$ & Zera todos floats de $x$ \\
\hline ov_getzerof () & Retorna um vetor de zeros \\
\hline ov_setf(escalar) & $\begin{array}{l}\text { Retorna um vetor com o escalar } \\
\text { repetido em todas as posições }\end{array}$ \\
\hline$o v \_\operatorname{maxf}(x, y)$ & Retorna o máximo por elemento \\
\hline ov_minf $(\mathrm{x}, \mathrm{y})$ & Retorna o mínimo por elemento \\
\hline ov_sqrtf $(x)$ & $\sqrt{x}$ \\
\hline ov_rsqrtf $(\mathrm{x})$ & $\frac{1}{\sqrt{x}}$ \\
\hline ov_sqrf $(x)$ & $x^{2}$ \\
\hline ov_rcpf $(\mathrm{x})$ & $\frac{1}{x}$ \\
\hline ov_floorf $(x)$ & $\lfloor x\rfloor$ \\
\hline ov_ceilf(x) & $\lceil x\rceil$ \\
\hline ov_maddf $(x, y, z)$ & $x \times y+z$ \\
\hline ov_msubf $(x, y, z)$ & $x \times y-z$ \\
\hline ov_absf (x) & $|x|$ \\
\hline
\end{tabular}

Tabela 2.2: Intrínsecos OpenVec para precisão simples.

Alguns intrínsecos OpenVec não têm uma instrução de hardware equivalente em todas as arquiteturas, por isso implementamos alguns intrínsecos OpenVec com um ou mais intrínsecos de hardware, ou em último caso, com código escalar. Por exemplo, implementamos o intrínseco multiplicar e somar ov_maddf no backend Intel SSE com dois intrínsecos proprietários:

\#define ov_maddf $(a, b, c)$ _mm_add_ps $\left(c,{ }_{-} m_{-} m_{-} m u l_{-} p s(a, b)\right)$

O entendimento das funções matemáticas é trivial, por exemplo a função ov_sqrtf $(\mathrm{x})$ calcula em paralelo a raiz quadrada para todos os floats de $\mathrm{x}$.

Os desenvolvedores que utilizam o OpenVec podem modificar e criar novas funções, baseadas em necessidades de desempenho e precisão. Em 
alguns backends não existe a instrução divisão vetorial, e varias instruções são necessárias para efetuar uma divisão. O exemplo a seguir cria uma divisão customizada, mais rápida e menos precisa que a divisão padrão do OpenVec.

$$
\text { \#define fast_div(x,y) ov_mulf(x,ov_rcpf }(y)) / / x \times \frac{1}{y}
$$

Os compiladores permitem relaxar a precisão para obtenção de maior desempenho com flags de otimização, mas somente a nível de arquivo de código fonte. O OpenVec traz a habilidade de customizar instruções ao nível de operação. Por exemplo, em um código com mil linhas o desenvolvedor pode customizar apenas uma divisão, em uma determinada porção do código.

\subsection{4}

\section{Vetorização de código condicional}

O conceito básico da vetorização é executar a mesma instrução em todos os elementos do vetor, mas em muitos casos existe um caminho divergente causado por um if-then-else. Nestes casos é preciso computar o código do if e o código do else para todos os elementos e juntar o resultado computado de cada código em um vetor.

A Figura 2.6 apresenta as operações intermediárias executadas pelo processador em um código SIMD condicional do tipo if-then-else. Em uma etapa inicial, é necessário computar os seguintes operandos da Figura 2.6:

- Máscara de bits (em verde) com os bits ligados nos elementos onde a condição do if é verdadeira

- Máscara de bits inversa (em laranja) com os bits ligados nos elementos onde a condição do if é falsa (else)

- Um vetor contento o resultado do bloco de código do then (em azul)

- Um vetor contendo o resultado do bloco de código do else (em vermelho)

Para agregar os resultados do then e do else em um único vetor, aplicamos uma operação AND entre a máscara do if (em verde) e o resultado do then (em azul), esta operação zera os elementos onde a condição do if é falsa. Também aplicamos uma operação AND entre a máscara inversa (em laranja) e o resultado do else (em vermelho), mantendo apenas os elementos onde a condição do else é verdadeira. Na última etapa, agregamos os resultados das duas operações AND com uma operação OR, resultando em um vetor com o then aplicado nos elementos onde a condição do if é verdadeira e o else aplicado nos elementos onde a condição do if é falsa. 


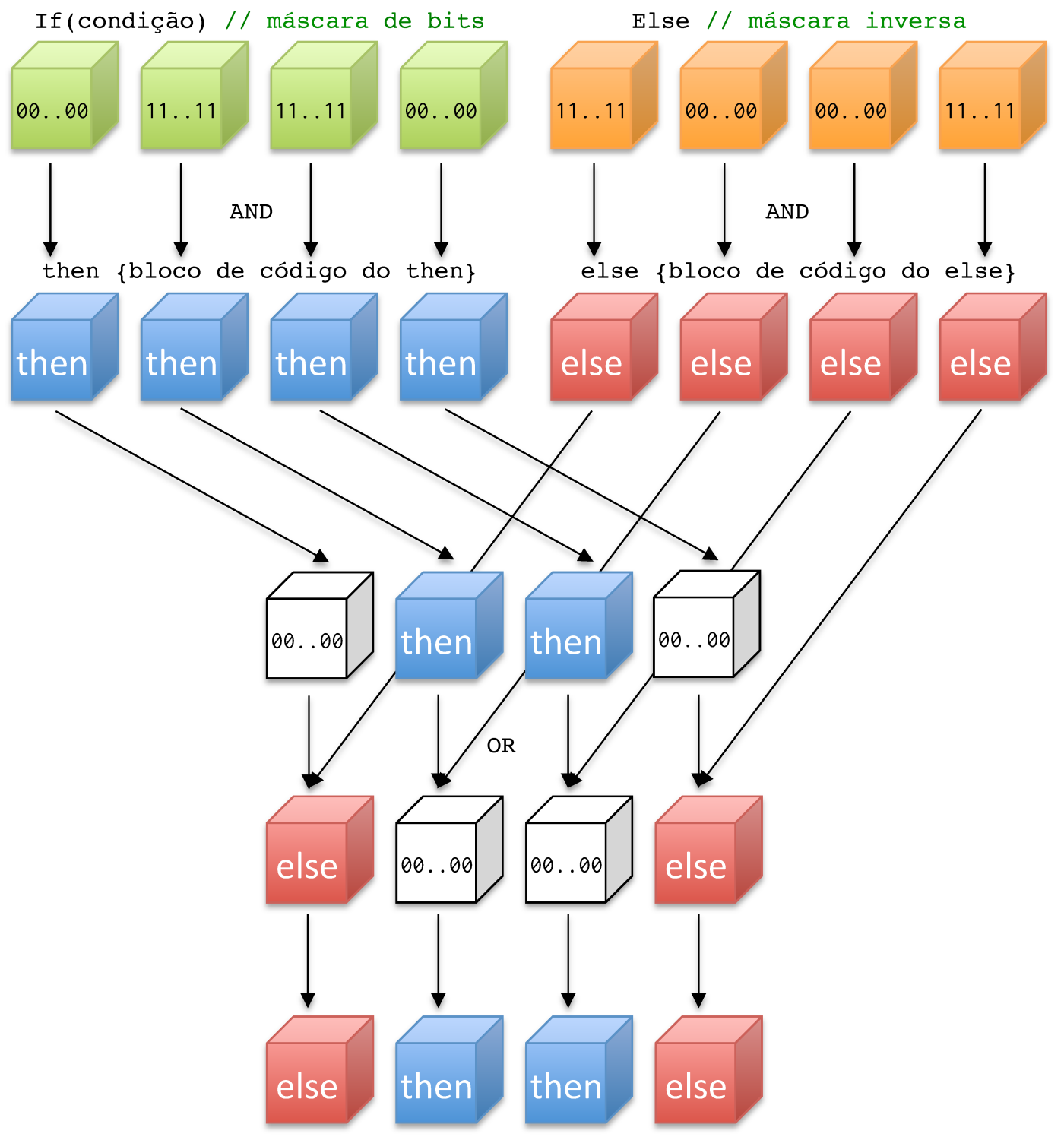

Figura 2.6: Operações intermediárias na vetorização de código condicional. Neste exemplo, a condição do if é verdadeira apenas para o segundo e o terceiro elementos.

A Listagem 2.4 mostra um simples if-then-else em um código escalar e a Listagem 2.5 mostra o código $\mathrm{C}++$ equivalente que vetoriza o if-then-else com a função ov_conditionalf.

Listagem 2.4: Simples if-then-else escalar.

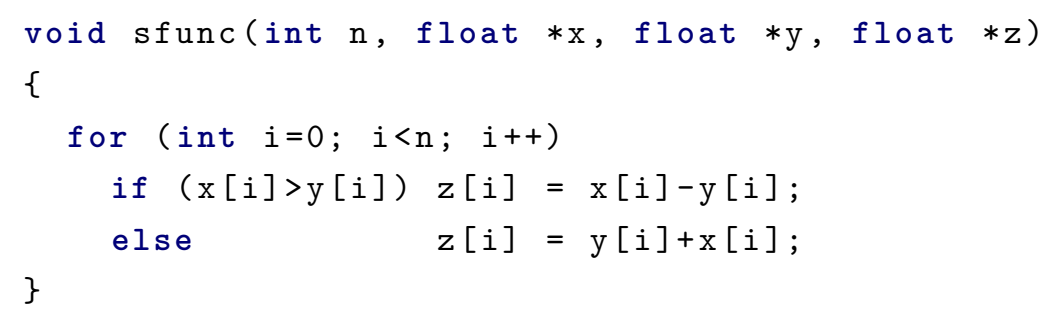


Listagem 2.5: Vetorização do if-then-else com a função ov_conditionalf.

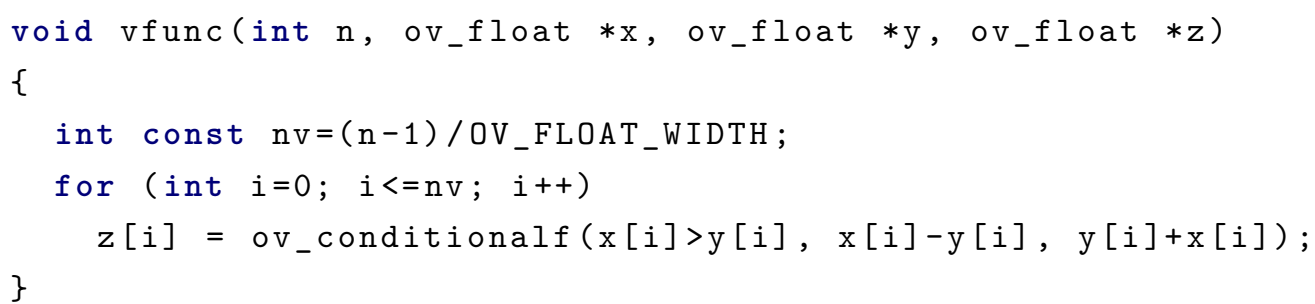

Implementamos em $\mathrm{C}++$ o overload de todos os operadores relacionais, conforme a Tabela 2.3. A operação $\mathrm{x}$ [i] >y [i] da Listagem 2.5 gera a máscara de bits, que é utilizada para juntar os resultados do if-then-else.

Com o OpenVec, não podemos codificar comandos if diretamente. A função ov_conditionalf recebe no primeiro argumento a máscara com os bits ligados para os elementos onde o resultado do teste é verdadeiro (em verde na Figura 2.6). O segundo argumento é a expressão a ser computada no caso do teste ser verdadeiro (then) e o terceiro argumento é a expressão a ser computada no caso do teste ser falso (else). O retorno dessa função é um vetor com o resultado da expressão 'then' nos elementos onde o if é verdade e com o resultado da expressão 'else' nos demais elementos.

Nem todos os processadores SIMD possuem uma instrução equivalente ao ov_conditionalf. Em algumas arquiteturas, necessitamos de múltiplas instruções para codificar um if. Estas instruções executam as operações da Figura 2.6. A seguir apresentamos nossa implementação para algumas arquiteturas:

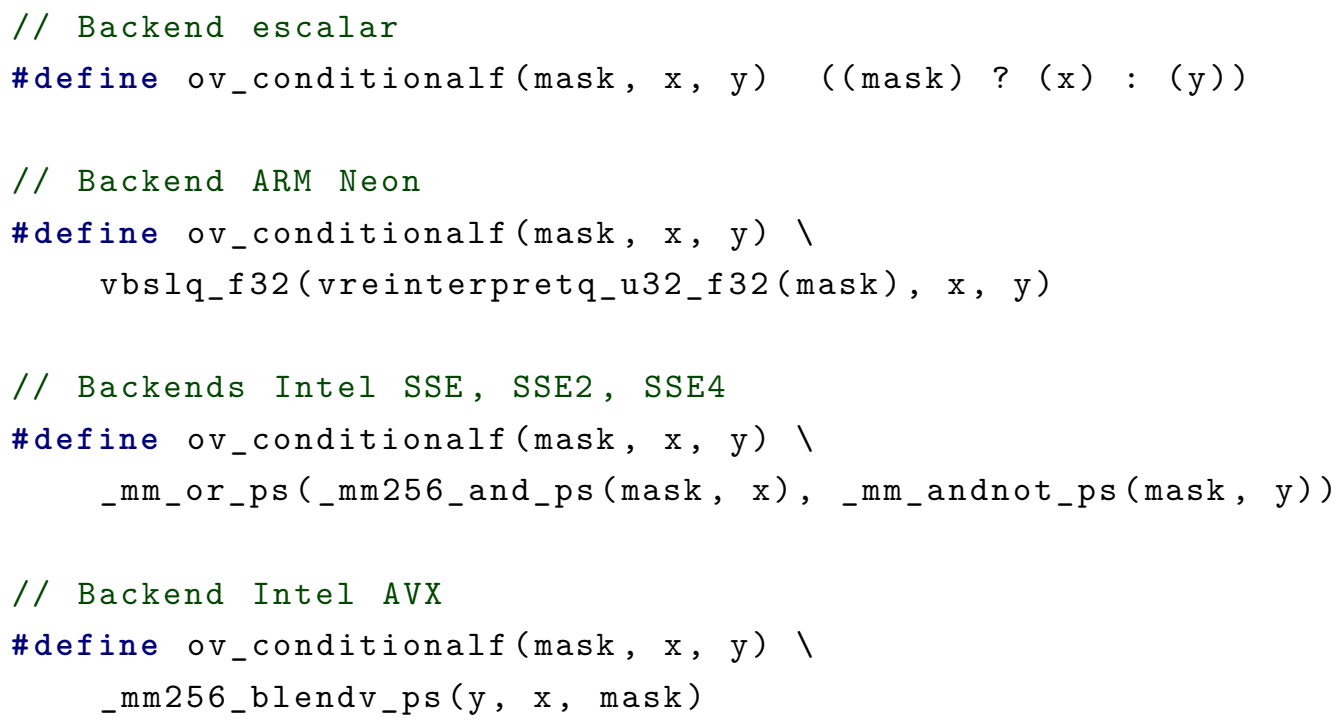

Em C, como não é possível fazer o overload dos operadores de comparação, criamos funções de comparação que retornam a máscara de bits. A Tabela 2.3 mostra essas funções, que também estão disponíveis em $\mathrm{C}++$. 


\begin{tabular}{|c|c|c|}
\hline Intrínseco de Comparação & Operador Relacional C++ & Descrição \\
\hline ov_eqf $(\mathrm{x}, \mathrm{y})$ & $==$ & $x=y$ \\
\hline ov_nef $(\mathrm{x}, \mathrm{y})$ & $\mathrm{!}=$ & $x \neq y$ \\
\hline ov_gtf $(\mathrm{x}, \mathrm{y})$ & $>$ & $x>y$ \\
\hline ov_gef $(\mathrm{x}, \mathrm{y})$ & $>=$ & $x \geq y$ \\
\hline ov_ltf $(\mathrm{x}, \mathrm{y})$ & $<$ & $x<y$ \\
\hline ov_lef $(\mathrm{x}, \mathrm{y})$ & $<=$ & $x \leq y$ \\
\hline
\end{tabular}

Tabela 2.3: Intrínsecos de comparação para precisão simples e operadores relacionais (apenas $\mathrm{C}++$ ). Esses intrínsecos retornam uma máscara de bits com os bits ligados nos elementos onde a comparação é verdadeira.

A Figura 2.7 mostra em detalhes a criação da máscara pela operação $\mathrm{x}>\mathrm{y}$ $(\mathrm{C}++)$ e a função ov_gtf $(\mathrm{x}, \mathrm{y})$. A comparação é feita em paralelo, de forma independente para cada elemento dos operandos vetoriais $\mathrm{x}, \mathrm{y}$.

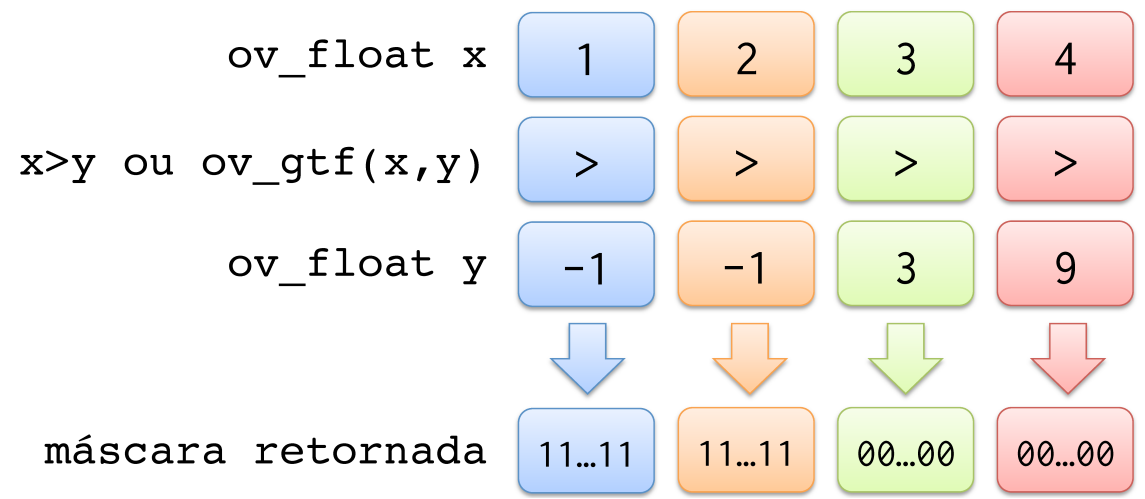

Figura 2.7: Criação da máscara de bits em um processador com uma unidade SIMD com largura OV_FLOAT_WIDTH=4.

Os operadores e as funções de comparação da Tabela 2.3 retornam o tipo ov_maskf ou ov_maskd (precisão dupla). Mapeamos esses tipos para os tipos proprietários de cada arquitetura.

O desenvolvedor não deve assumir que o tamanho do tipo ov_maskf é igual ao tamanho do tipo ov_float. Em algumas arquiteturas a máscara utiliza apenas um bit por elemento e em outras utiliza a mesma quantidade de bits do tipo que está sendo comparado. Por exemplo, em uma arquitetura Intel SSE uma variável vetorial do tipo ov_float possui 128 bits (4x32) e a máscara também possui 128 bits, enquanto a arquitetura Intel AVX-512 utiliza apenas 16 bits para representar uma máscara resultado de uma comparação de 16 floats (512 bits). 


\subsection{5}

\section{Otimização da vetorização de código condicional}

Em alguns casos, em apenas alguns elementos a execução entra no caminho do código do else ao invés do then. Este comportamento é muito comum em simulações numéricas, onde determinadas operações devem ser aplicadas apenas nas bordas do modelo. Neste caso a vetorização com máscara pode ser muito ineficiente pois ambos os blocos de código do then e do else são executados. Mapeamos intrínsecos que verificam se todos os elementos de um vetor SIMD vão percorrer o caminho do then ou do else, para que apenas um dos dois códigos seja executado e não seja necessário aplicar a máscara.

Criarmos duas funções básicas, uma que verifica se todos os bits da máscara estão ligados, e outra que verifica se pelo menos um bit está ligado. Essas funções fazem a redução de todos os elementos da máscara para um valor escalar, que pode ser verdadeiro ou falso, conforme a Tabela 2.4.

\begin{tabular}{|l|r|}
\hline Intrínseco de redução & Descrição \\
\hline ov_allf(máscara) & $\begin{array}{c}\text { Retorna um valor diferente de zero } \\
\text { se a máscara é verdade para todos os elementos }\end{array}$ \\
\hline ov_anyf(máscara) & $\begin{array}{r}\text { Retorna um valor diferente de zero } \\
\text { se a máscara é verdade para pelo menos um elemento }\end{array}$ \\
\hline
\end{tabular}

Tabela 2.4: Intrínsecos de redução para máscaras de comparação entre objetos do tipo ov_float. Esses intrínsecos também estão disponíveis para precisão dupla (sufixo "d").

Com essas funções podemos otimizar a vetorização de códigos condicionais, testando se todos os elementos entram no bloco do if ou do else, se todos os elementos entram no mesmo caminho de código então não é necessário aplicar a máscara, a Listagem 2.6 mostra a otimização do código vetorial da Listagem 2.5.

Listagem 2.6: Otimização da vetorização do if-then-else com a função ov_allf.

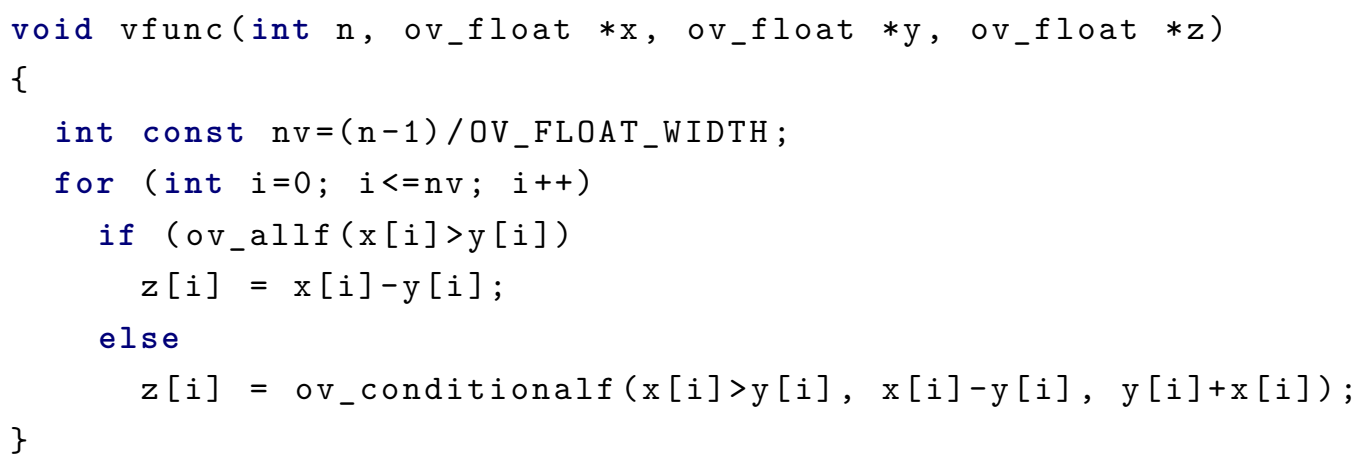


No código da Listagem 2.6, se todos os elementos do vetor SIMD x [i] são maiores que os respectivos elementos de y [i], então o código do else não é computado e nenhuma máscara é aplicada. Senão, a versão mais lenta com a aplicação da máscara é executada, computando ambos os blocos de código do if e do else, mostrados na Listagem 2.4.

O tipo de retorno das funções da Tabela 2.4 varia para cada backend, mas o desenvolvedor pode assumir que o valor retornado pode ser utilizado na avaliação de um if, conforme o exemplo a seguir:

if $\left(o v_{-} a l l f(\operatorname{mascara})\right) \ldots$

Decidimos não incorporar as funções de redução de máscara na função ov_conditionalf. Acreditamos que cabe ao o desenvolvedor decidir se o then ocorre com mais frequência que o else, ou vice versa.

Algumas arquiteturas possuem uma instrução que permite testar se os elementos de um vetor SIMD são negativos ou não, testando apenas o bit do sinal, sem a necessidade de gerar uma máscara de comparação. Implementamos os intrínsecos da Tabela 2.5 que tiram proveito dessa funcionalidade. Esses intrínsecos testam se todos ou se alguns elementos de um vetor SIMD são negativos ou não.

\begin{tabular}{|l|c|}
\hline Intrínseco de redução & Descrição \\
\hline ov_any_lt_of $(\mathrm{x})$ & $\begin{array}{c}\text { Retorna um valor diferente de zero } \\
\text { se pelo menos um elemento de } x<0\end{array}$ \\
\hline ov_any_ge_of $(\mathrm{x})$ & $\begin{array}{c}\text { Retorna um valor diferente de zero } \\
\text { se pelo menos um elemento de } x \geq 0\end{array}$ \\
\hline ov_all_lt_of $(\mathrm{x})$ & $\begin{array}{c}\text { Retorna um valor diferente de zero } \\
\text { se todos os elementos de } x<0\end{array}$ \\
\hline ov_all_ge_of $(\mathrm{x})$ & $\begin{array}{c}\text { Retorna um valor diferente de zero } \\
\text { se todos os elementos de } x \geq 0\end{array}$ \\
\hline
\end{tabular}

Tabela 2.5: Intrínsecos de redução para o testar se os elementos de um objeto ov_float são negativos. Esses intrínsecos também estão disponíveis para precisão dupla (sufixo "d").

Nos backends onde essas instruções de teste de bit do sinal não existem, implementamos esses intrínsecos com a combinação de uma função de comparação com uma função de redução, conforme o exemplo a seguir:

\#define ov_any_lt_of(x) ov_anyf(ov_ltf(x, ov_getzerof( $))$ ) 
Como no backend escalar só existe um elemento por vetor, as funções de redução condicional do tipo ov_any e ov_all são implementadas da mesma forma. A seguir a implementação escalar das funcções ov_any_lt_0f e ov_all_lt_of:

\#define ov_any_lt_of $(x) \quad((x)<0.0 f)$

\#define ov_all_lt_of ov_any_lt_of

\subsection{6}

\section{Tratamento da cauda dos vetores}

Até o momento, em todos os exemplos de código que apresentamos, não levamos em conta se o número de iterações dos loops era múltiplo de OV_FLOAT_WIDTH. Nesta Seção tratamos o caso onde o número de iterações não é múltiplo de OV_FLOAT_WIDTH.

Uma estratégia para garantir que os loops tenham o número de iterações múltiplo de OV_FLOAT_WIDTH é fazer com que a dimensão dos arrays que é contígua em memória seja múltipla de OV_FLOAT_WIDTH. Essa estratégia também garante um alinhamento de memória (e também é utilizada para otimizar o acesso à memória na programação geral em GPUs, com indexação a partir de um endereço base alinhado e offsets múltiplos de CUDA warp size[37] ou CL_KERNEL_PREFERRED_WORK_GROUP_SIZE_MULTIPLE no caso de OpenCL[38]).

Se alocamos um array com a função ov_malloc, então podemos deixar o loop "invadir"os últimos elementos do vetor na última iteração, pois a função ov_malloc aloca OV_FLOAT_WIDTH elementos adicionais. Na Listagem 2.1, podemos notar que a última iteração processa elementos adicionais. Esta condição ocorre quando o número $\mathrm{n}$ de iterações não é múltiplo de OV_FLOAT_WIDTH.

Essa estratégia de invadir os últimos elementos pode ser vista na Listagem 2.1 e contempla a maioria dos casos de uso. Mas, em alguns casos como por exemplo, na atualização de apenas uma faixa dos elementos de um array, os limites dos loops devem ser respeitados para a corretude do código.

A Listagem 2.7 mostra uma cópia de memória SIMD entre dois arrays do tipo float, onde o limite $n$ do loop é respeitado.

Criamos a constante OV_FLOAT_TAIL com o valor OV_FLOAT_WIDTH-1. Podemos ver na Listagem 2.7 que o primeiro loop subtrai OV_FLOAT_TAIL elementos do limite $n$ do loop, garantindo que o limite $n$ elementos não é excedido. 
Listagem 2.7: Exemplo de uma cópia de floats entre arrays.

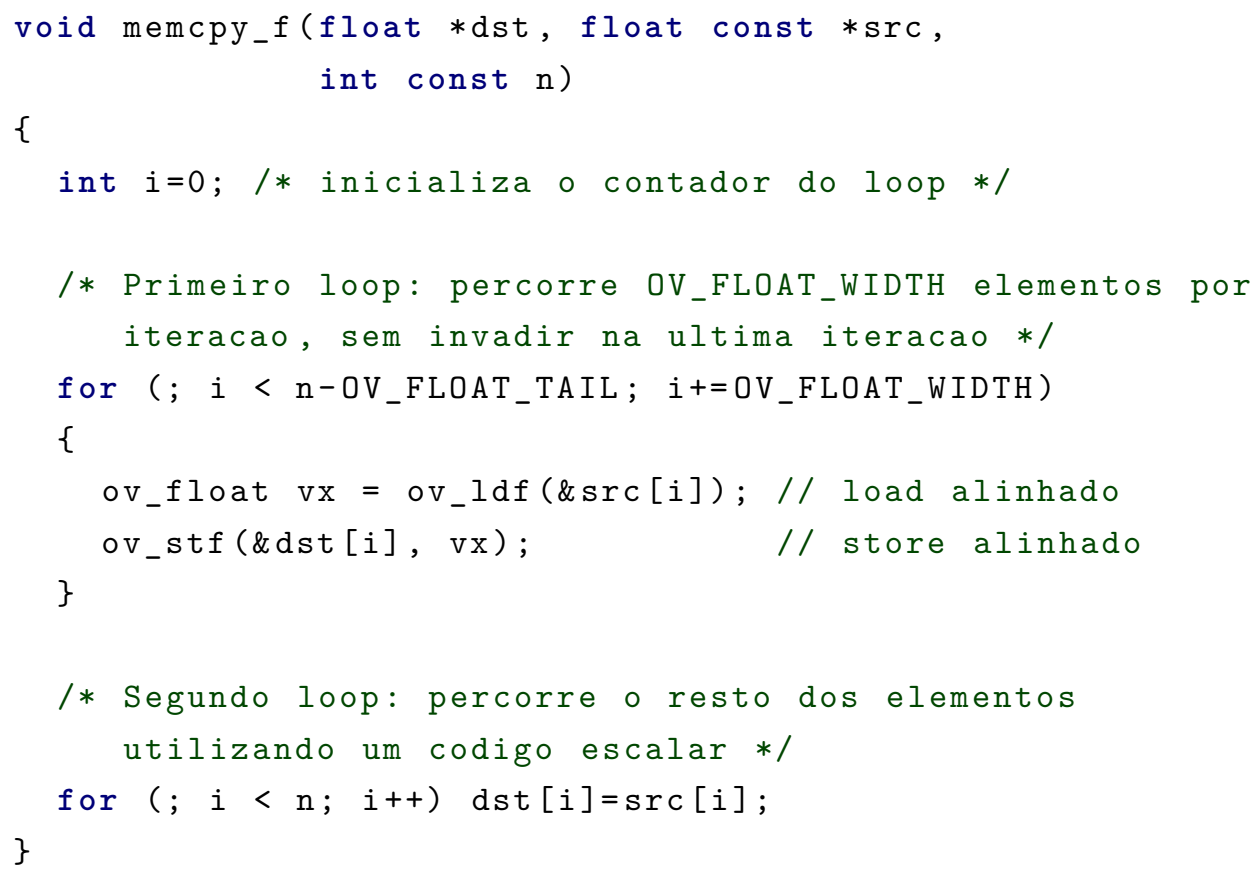

\subsection{7 \\ Reduções}

Operações de redução são frequentes em aplicações de alto desempenho, onde é comum fazer operações entre os elementos de uma variável vetorial, como por exemplo calcular a soma de todos os elementos de um array. O OpenVec provê funções matemáticas de redução como a função ov_all_sumf que recebe uma variável SIMD do tipo ov_float e retorna um único float como resultado, conforme a demonstração abaixo para uma arquitetura com 8 elementos por vetor SIMD:

$$
\text { retorno } \leftarrow x_{0}+x_{1}+x_{2}+x_{3}+x_{4}+x_{5}+x_{6}+x_{7}
$$

A Listagem 2.8 mostra um código que soma todos os elementos de um array, tratando o caso em que o número de elementos do array não é múltiplo de OV_FLOAT_WIDTH. Este código também faz o tratamento de cauda.

Listagem 2.8: Soma de todos os elementos de um array

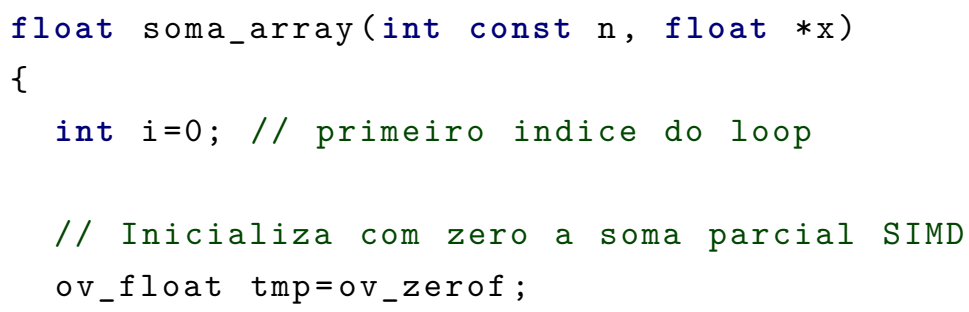




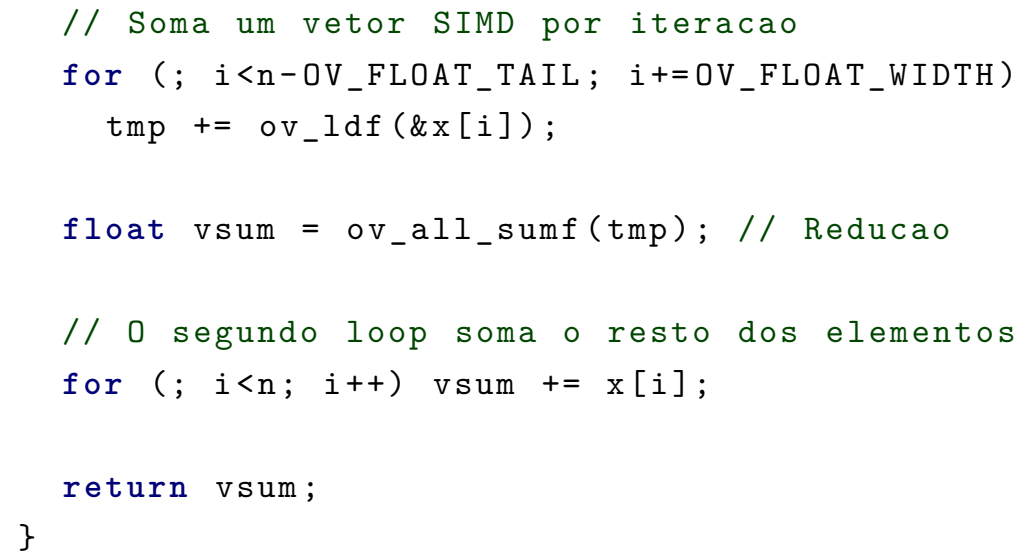

Os resultados da soma da Listagem 2.8 podem variar entre as diferentes arquiteturas, porque a ordem em que os floats são somados influencia o resultado. Por exemplo, somar um valor muito pequeno a um valor muito grande não modifica o valor muito grande nos casos onde a diferença entre eles é maior que a precisão da mantissa.

Nossa implementação corrente não implementa as funções de redução matemática de forma otimizada. Elas são implementadas com um código escalar para todas as arquiteturas. Mas tendo em vista que essas funções são normalmente utilizadas para juntar os resultados após um loop, o impacto no desempenho é irrelevante.

A Tabela 2.6 mostra as funções de redução matemática.

\begin{tabular}{|l|c|}
\hline Função & Operação \\
\hline ov_all_sumf (x) & $x_{0}+x_{1}+x_{2}+\ldots+x_{n}$ \\
\hline ov_all_prodf (x) & $x_{0} \times x_{1} \times x_{2} \times \ldots \times x_{n}$ \\
\hline ov_all_maxf(x) & $\max \left(x_{0}, x_{1}, x_{2}, \ldots, x_{n}\right)$ \\
\hline ov_all_minf(x) & $\min \left(x_{0}, x_{1}, x_{2}, \ldots, x_{n}\right)$ \\
\hline
\end{tabular}

Tabela 2.6: Funções de redução matemática, com $n=$ OV_FLOAT_WIDTH-1. O OpenVec implementa as mesmas funções para precisão dupla com o sufixo $d$.

\section{2}

\section{Discussão}

Nossa implementação mantem o "look and feel" da programação proprietária com intrínsecos, mas de maneira portável. Para manter o desempenho não adicionamos nenhum grau de abstração, tornando talvez, o seu uso mais difícil.

Como o OpenVec trata apenas a vetorização, o desenvolvedor deve utilizar uma outra ferramenta para utilizar todos os núcleos da CPU. A Seção 
4.6 apresenta uma avaliação de desempenho do OpenVec em conjunto com o OpenMP.

Em $\mathrm{C}++$, nosso overload de operadores facilitou muito a vetorização explícita em relação aos intrínsecos proprietários.

Poderemos no futuro, adicionar ao OpenVec todos os intrínsecos de todas as arquiteturas suportadas. E prover ao desenvolvedor uma forma de verificar se o intrínseco OpenVec é nativo ou emulado. Esta verificação é feita em tempo de de compilação (ex: \#ifdef OV_NATIVE_SHUFFLE). Então, o desenvolvedor pode escrever algumas versões de código e combinar esta funcionalidade com uma estratégia de off-line autotuning (em tempo de compilação)[39].

Esta abordagem traz a desvantagem da manutenção de múltiplas versões de código. E traz a vantagem da possibilidade de atingir todo o desempenho potencial de uma arquitetura, tendo em vista que todos os seus intrínsecos nativos estão disponíveis.

Recomendamos que quando possível, o desenvolvedor utilize a keyword restrict para informar ao compilador que os ponteiros não estão em alias. $\mathrm{O}$ uso do restrict pode ajudar o compilador a fazer algumas otimizações[40]. Como o restrict não faz parte do padrão C original, essa keyword pode variar entre compiladores. Fornecemos a keyword ov_restrict que mapeia para o restrict específico de todos os compiladores que utilizamos neste trabalho.

Disponibilizamos o OpenVec sob a licença MIT[41]. O projeto pode ser baixado no endereço https://github.com/OpenVec/OpenVec. 


\section{3}

\section{Biblioteca de Arquiteturas Heterogêneas}

Após portar aplicações de imageamento sísmico para arquiteturas heterogêneas tais como IBM Cell[21] e Nvidia CUDA[42][43][44], notamos que um grande esforço de desenvolvimento é gasto no gerenciamento dos dispositivos secundários, ou seja, em um código de preparação do dispositivo para poder executar as operações de interesse. Isso nos motivou a implementar uma biblioteca que provê as tarefas usuais na programação em um ambiente heterogêneo, composto por um processador principal e um ou mais dispositivos auxiliares de processamento. Por questões históricas, implementamos esta biblioteca em Fortran 90. Neste capítulo descrevemos esta biblioteca.

O modelo de computação heterogênea, muitas vezes possui dois tipos de memória: a memória principal, que é conectada ao processador (CPU) e que neste contexto definimos como memória da CPU ou host memory e a memória de cada dispositivo. A Figura 3.1 mostra um exemplo de arquitetura heterogênea do tipo CPU+GPU.

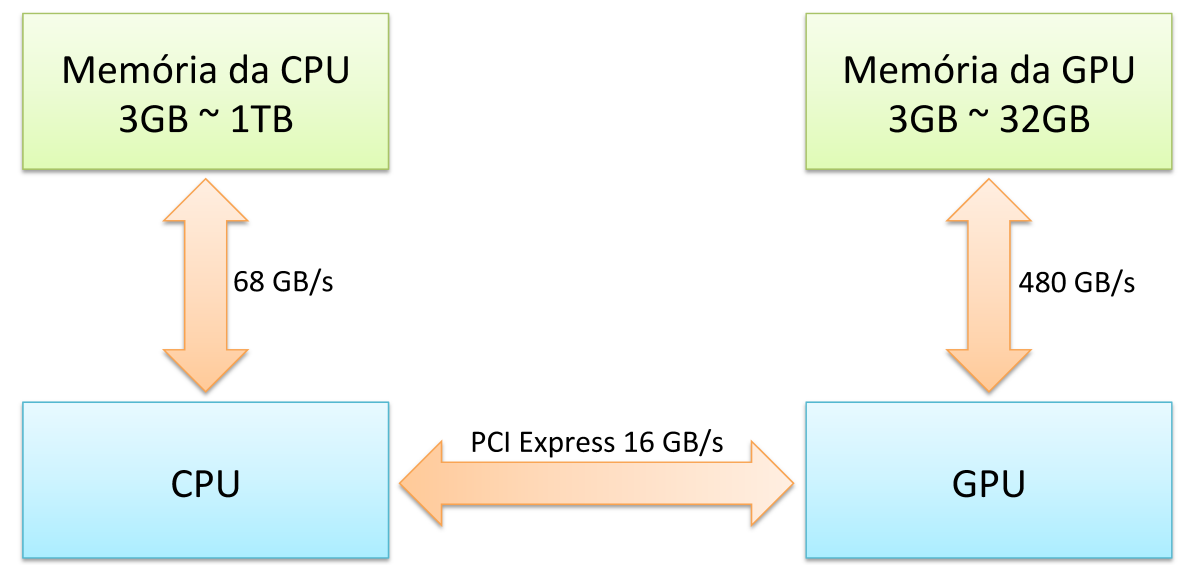

Figura 3.1: Esquema de conexão em uma arquitetura heterogênea CPU+CPU. Desempenho de conexão baseado na seguinte configuração: CPU Xeon E5-4669 v3, GPU Nvidia K80 conectada via PCIe 16X de $3^{\text {a }}$ geração.

\section{1}

\section{Programação de Arquiteturas Heterogêneas}

Para tirar proveito de uma arquitetura heterogênea, o programador deve dividir sua aplicação em dois tipos de código, um que é executado no processador principal (CPU) e outro que é executado em um dispositivo secundário, como por exemplo GPU. Esses dispositivos também são chamados de aceleradores e não funcionam de forma independente, precisando estar conectados a um processador principal. Neste trabalho usaremos o termo CPU para definir o processador principal. Apesar dos dispositivos secundários possuírem uma 
ou mais unidades de processamento, usaremos o termo dispositivo para definir o dispositivo auxiliar, que é diferente do processador principal.

O interesse em utilizar esses dispositivos vem de fatores variados[45], como maior desempenho, economia de energia e espaço ou simplesmente melhor custo-benefício. A Figura 3.2 (fonte: CUDA C Programming Guide[46]) compara a evolução do poder computacional das placas gráficas Nvidia (dispositivo secundário) com as CPUs Intel.

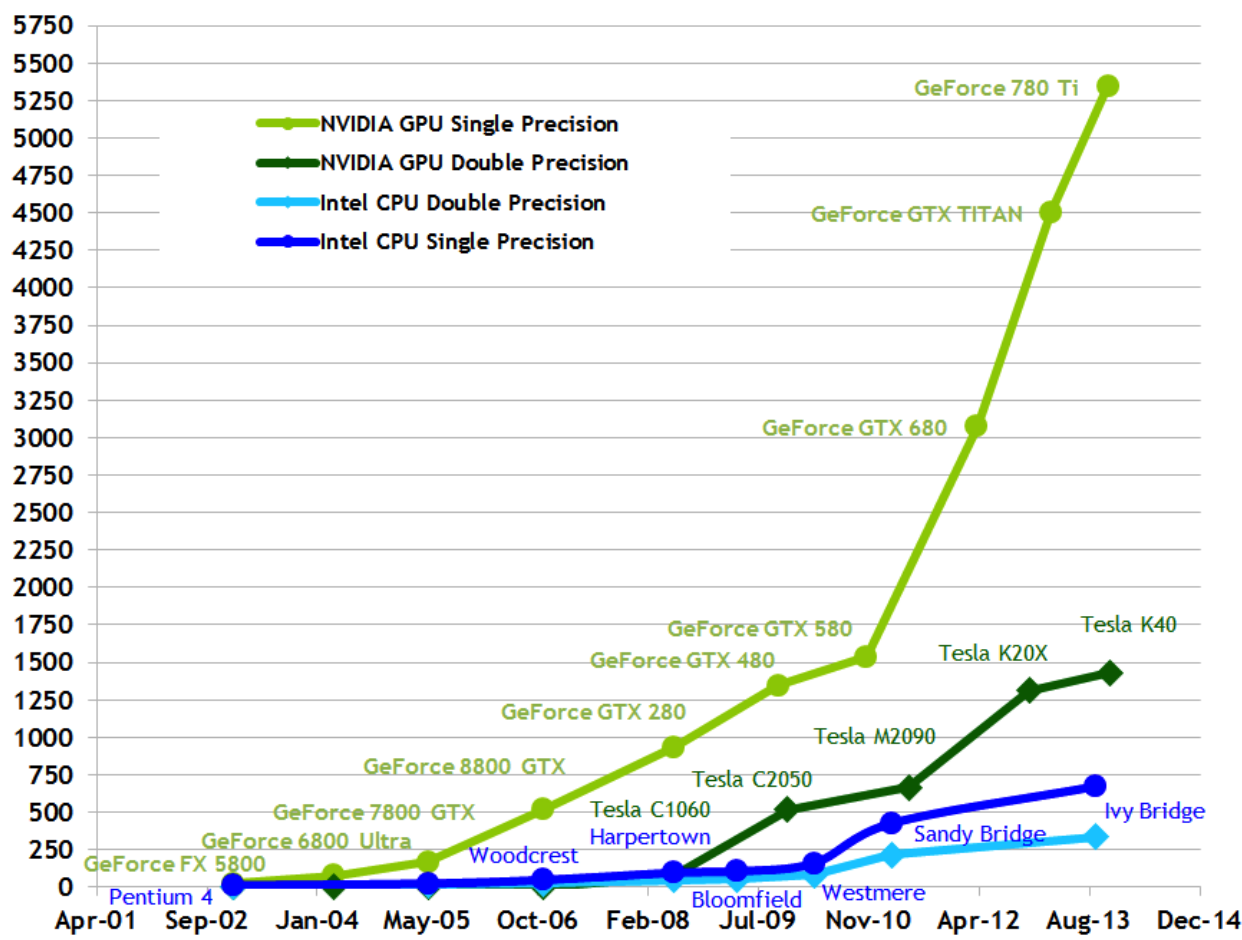

Figura 3.2: Comparativo da evolução do poder computacional em GFlops/s entre Nvidia e Intel. Computação heterogênea em verde, computação homogênea em azul.

O código que é executado na CPU compreende a inicialização da aplicação, E/S e a interface com usuário. Na computação heterogênea, o código da CPU também faz o gerenciamento dos dispositivos, como por exemplo alocação de memória no dispositivo. Os dispositivos executam pequenas porções de código, tipicamente chamados de kernels.

Para executar um kernel no dispositivo, o desenvolvedor precisa inicializar o dispositivo, alocar uma área de memória no dispositivo e provavelmente fazer a inicialização desta área, copiando o conteúdo da memória principal (memória da CPU) para essa memória. A memória alocada no dispositivo é passada como argumento no momento da invocação do kernel. O código que executa na CPU é o responsável por fazer a invocação de um kernel no dispositivo, a inicialização do dispositivo, a alocação / inicialização da memória do dispositivo e por controlar as filas de submissão. Diversos trabalhos[47, 48, 49] 
consideram esse código de gerenciamento que executa na CPU como sendo um "boilerplate code".

Como existe uma memória para a CPU e outra memória para o dispositivo, o desenvolvedor deve escolher com cuidado onde colocar cada estrutura de dados. Sua escolha deve se basear na velocidade e na capacidade de cada memória e na velocidade da conexão entre elas. A conexão entre a CPU e o dispositivo é feita pelo barramento PCI Express ${ }^{1}$ que atualmente é o gargalo deste tipo de arquitetura, pois sua banda passante é muito menor que as bandas das memórias da CPU e do dispositivo.

O tempo gasto nas transferências $\mathrm{CPU} \Rightarrow$ dispositivo e dispositivo $\Rightarrow \mathrm{CPU}$ inviabiliza a implementação eficiente de muitos algoritmos. Um determinado kernel pode executar em menos tempo no dispositivo se comparado com sua versão que executa na CPU, mas o tempo gasto para transferir os argumentos de entrada e saída pode cancelar o ganho de desempenho que este kernel traria. Entretanto, é possível adicionar uma concorrência interna ao dispositivo e entre dispositivos, sobrepondo computação e transferências, minimizando o impacto do tempo de transferência.

Durante o processo de desenvolvimento, o desenvolvedor gasta muito tempo com o código de gerenciamento, que faz a preparação para a execução no kernel, como por exemplo, cópias entre as memórias da CPU e do dispositivo. Usualmente não é possível isolar esse código de gerenciamento do dispositivo em uma parte específica do código. Além disso é preciso tratar o código da arquitetura convencional, que só executa na CPU. Com isso o código fica com tratamentos de diferentes arquiteturas espalhados por diferentes trechos, ficando difícil de ler e manter.

O kernel normalmente é o hot spot da aplicação i.e. o trecho de código com o qual se pretende reduzir o tempo de execução. Mas em alguns casos, o desenvolvedor gasta mais esforço[50] e linhas de código gerenciando dispositivos e mantendo duas ou mais versões do código do que atacando o problema de aumentar o desempenho do hot spot da aplicação.

Diferentemente dos kernels, que podem ser isolados em uma invocação de função, o código de gerenciamento aparece em diferentes partes/camadas da aplicação, o que torna difícil desacoplar esse código da aplicação. O processo de adaptação e tuning de um código para uma arquitetura heterogênea como CUDA ou OpenCL pode ser demorado, envolver uma significativa reorganização do código e ficar mais suscetível a erros[51].

${ }^{1}$ PCI Express (Peripheral Component Interconnect Express) é o barramento padrão para conexão de dispositivos, tais como, placas de rede, controladoras de disco, placas gráficas (GPUs) e aceleradores. 
Abordagens como OpenMP 4.0[52], OpenACC[53], OCCA[54] e HMPP [55], que utilizam um único código portável com anotações, ainda não atingem o desempenho obtido ao codificar diretamente com a API mais adequada a cada dispositivo[56, 57, 58, 59]. Ou seja, para se obter o maior desempenho em uma determinada arquitetura é preciso utilizar a API que melhor se adapta àquela arquitetura. Muitas vezes essa API é proprietária, como é o caso das APIs Nvidia CUDA e IBM AltiVec intrinsics.

Para permitir que o desenvolvedor se concentre no hot spot da aplicação, implementamos a biblioteca HLIB[60, 61] que gerencia as primitivas básicas da computação heterogênea mas que também funciona, de forma transparante, com arquiteturas homogêneas tradicionais (somente CPU). A biblioteca implementa os aspectos da computação heterogênea, como configurações e inicializações, mas deixa a cargo do desenvolvedor implementar o kernel de forma nativa, por exemplo, em CUDA, ou utilizando OpenMP em conjunto com SIMD intrinsics da Intel.

A biblioteca HLIB tem como público alvo desenvolvedores que desejam utilizar computação heterogênea utilizando APIs que não comprometem o desempenho. O ganho com o uso da biblioteca é aumentar o reuso de código, já que seu uso não traz nenhum ganho de desempenho, pois cabe ao desenvolvedor codificar os kernels de forma nativa para cada arquitetura.

A biblioteca fornece a liberdade de codificar os kernels na API que apresenta o maior potencial de desempenho para determinada arquitetura. O foco do desenvolvimento passa a ser o hot spot da aplicação, pois com a HLIB todo o resto do código de gerenciamento que executa na CPU é portável e agnóstico de arquitetura.

A HLIB implementa todo o código de gerenciamento do dispositivo, que compreende as primitivas da Tabela 3.1.

Inicializar / finalizar dispositivo

Alocar / desalocar memória no dispositivo

Alocar / desalocar memória para transferências CPU $\Leftrightarrow$ dispositivo

Zerar memória alocada no dispositivo

Cópia de memória $\mathrm{CPU} \Leftrightarrow$ dispositivo e dispositivo $\Leftrightarrow$ dispositivo

Concorrência entre computação e cópias de memória

Passagem de mensagem entre dispositivos

Tabela 3.1: Primitivas HLIB da computação heterogênea.

A principal diferença entre a nossa abordagem e trabalhos similares[62, $63,64]$ é que, na nossa abordagem, cabe ao desenvolvedor enfileirar os kernels e invocar explicitamente as transferências entre a memória da CPU e a memória do dispositivo. Como apenas mapeamos funcionalidades já existentes, o uso da 
biblioteca não restringe a capacidade de atingir o desempenho possível em cada arquitetura e mantém uma porção crítica do código portável e reutilizável. Podemos classificar o grau de portabilidade na computação heterogênea em três níveis:

- Máximo: um código para todas as arquiteturas

- Médio: um código de gerenciamento e múltiplos kernels para várias arquiteturas

- Mínimo: múltiplos kernels e códigos de gerenciamento para várias arquiteturas

A Figura 3.3 faz um comparativo entre o desempenho e o grau de portabilidade. Com a HLIB existe um único código de gerenciamento mas é necessário portar o kernel para cada arquitetura. Com relação ao desempenho uma aplicação que utiliza a HLIB se localiza próxima às APIs nativas, pois o hot spot é codificado da mesma forma na HLIB e na API nativa. Classificamos com o grau médio de portabilidade uma aplicação que utiliza a HLIB.

\section{Portabilidade}

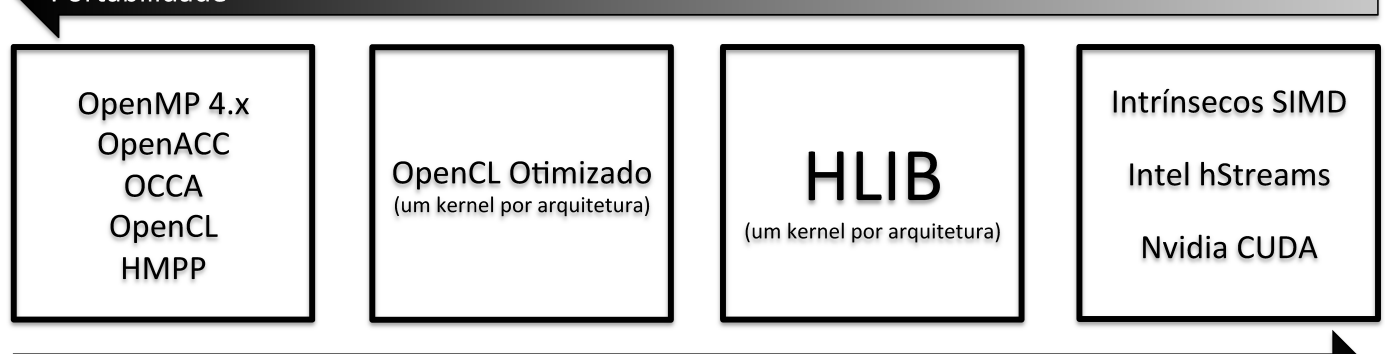

Desempenho Potencia

Figura 3.3: Comparativo entre o grau de portabilidade e desempenho potencial. OpenCL otimizado se refere a múltiplos kernels OpenCL otimizados para cada arquitetura. O desempenho é potencial, fica a cargo do desenvolvedor.

A Figura 3.4 mostra a organização de uma aplicação utilizando a HLIB. A biblioteca implementa todas a primitivas da Tabela 3.1.

Na Figura 3.4, a porção em vermelho representa a biblioteca heterogênea HLIB com os backends para as diversas plataformas suportadas: CUDA, OpenCL, hStreams e código regular homogêneo. As bibliotecas de runtime de cada arquitetura são mostradas em verde. A aplicação, em azul, contém duas camadas bem distintas: a camada de cima, que contém um código agnóstico de arquitetura e invoca a biblioteca heterogênea, e a camada de baixo que possui kernels com otimizações específicas para cada arquitetura.

Esses kernels são codificados de forma que sua invocação tenha a mesma interface Fortran 90: a aplicação invoca o kernel OpenCL da mesma forma 


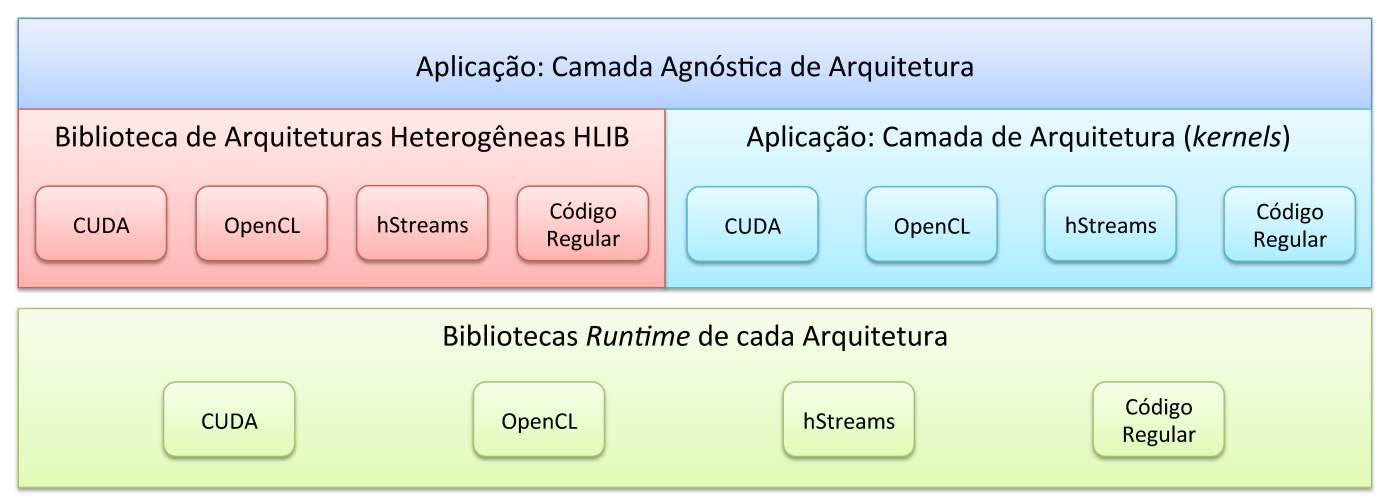

Figura 3.4: Aplicação em azul, biblioteca heterogênea em vermelho e as bibliotecas runtime de cada arquitetura em verde.

que invoca o kernel CUDA. Não existe nenhuma chamada CUDA ou qualquer chamada exclusiva de uma arquitetura espalhada pelo código da aplicação. Todas as chamadas proprietárias estão contidas nos kernels.

Em derterminadas APIs, como CUDA e OpenCL, a invocação dos kernels é feita utilizando sintaxes distintas e não pode ser feita diretamente por um código Fortran 90. Com isso, é necessário que o desenvolvedor crie um código driver com a mesma interface para todas as arquiteturas. O driver faz a invocação do kernel com a sintaxe apropriada de cada API. A Listagem 3.1 mostra o código de um driver escrito em CUDA para a função SAXPY. Este driver fica em um arquivo CUDA e é compilado com o compilador da Nvidia (nvcc).

\section{Listagem 3.1: Exemplo de um código driver}

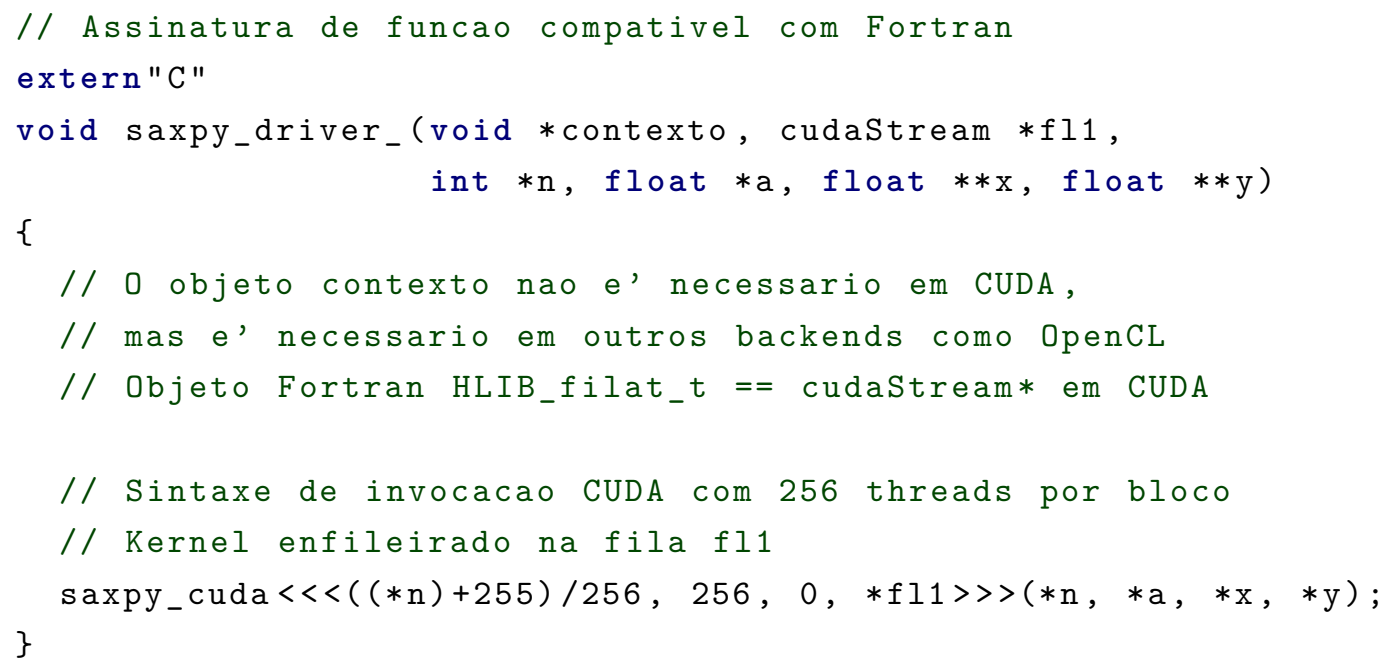

A camada agnóstica de arquitetura, que executa na CPU, invoca o driver. E o driver invoca o kernel no dispositivo. A seguir temos um exemplo da invocação Fortran do driver da Listagem 3.1:

CALL Saxpy_driver(contexto, fila, n, a, x, y) 
Esta invocação contempla todas as arquiteturas e faz parte da camada agnóstica. Cabe ao desenvolvedor escrever o driver e o kernel para cada arquitetura de interesse.

\section{2}

\section{Implementação}

Implementamos a biblioteca heterogênea de forma que um único código de gerenciamento possa ser utilizado da mesma forma, em arquiteturas homogêneas e heterogêneas. Implementamos backends para as seguintes arquiteturas:

- Nvidia CUDA

- OpenCL

- Intel hStreams

- CPU regular (arquitetura homogênea com C e Fortran)

A biblioteca é exposta com um módulo Fortran 90, e implementa uma interseção de funcionalidades de três APIs: Nvidia CUDA, OpenCL e Intel hStreams, conforme a Figura 3.5.

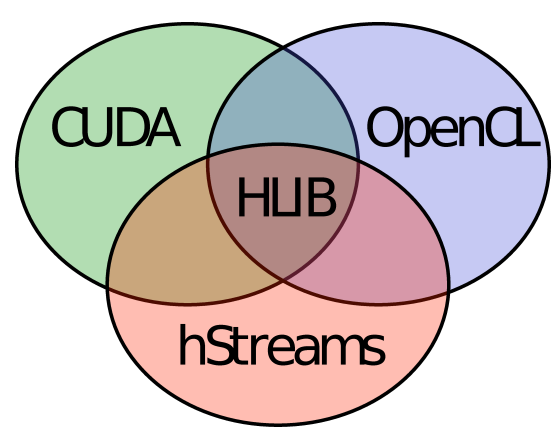

Figura 3.5: A HLIB implementa uma interseção de funcionalidades de diversas APIs.

Muitas decisões de implementação foram feitas para manter a compatibilidade com as APIs suportadas. Por exemplo, para manter a compatibilidade com o OpenCL, a memória alocada no dispositivo é um objeto opaco e não um ponteiro. Como outro exemplo, as filas de execução são sempre FIFO para garantir a compatibilidade com o cudaStream da Nvidia.

As funcionalidades da HLIB são muito próximas das funcionalidades das APIs dos backends. Em muitos casos, nossos backends apenas invocam uma única função correspondente da API da arquitetura. Por exemplo, invocamos a função cudaMemcpyAsync para implementar a primitiva de cópia de memória em CUDA. 
A interseção de funcionalidades da Figura 3.5 foi suficiente para implementarmos as primitivas da Tabela 3.1. Não houve perda de desempenho nestas primitivas, pois existe um mapeamento direto para as funcionalidades das APIs dos backends.

Abordamos na Seção 3.4 o uso de primitivas específicas de uma arquitetura, isto é, primitivas que não estão contidas na interseção de APIs da Figura 3.5 .

\subsection{1}

\section{Inicializar / finalizar dispositivo}

O primeiro passo ao se utilizar a HLIB é inicializar o dispositivo criando um contexto. Ao inicializar um dispositivo um objeto é retornado com o contexto criado com o dispositivo.

O contexto é um descritor do dispositivo, e após a inicialização fica associado a um único dispositivo. O contexto é utilizado durante a execução das primitivas da Tabela 3.1 para informar ao runtime da arquitetura qual dispositivo deverá executar a primitiva. Como exemplo, em OpenCL o contexto contém um objeto nativo do tipo cl_context. No momento da invocação o desenvolvedor informa o número do dispositivo que deseja utilizar, pois uma máquina pode ter mais de um dispositivo secundário. O código a seguir mostra a interface de criação de contexto com dispositivo:

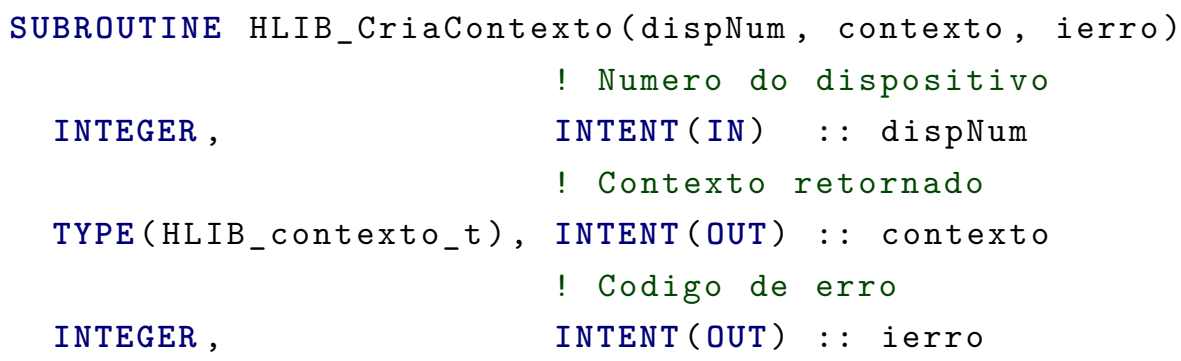

Implementamos o tipo HLIB_contexto_t como um objeto opaco de Fortran, ou seja, um objeto onde todos os atributos são privados.

Todas as demais sub-rotinas da biblioteca recebem como argumento o contexto, atuando no dispositivo associado a ele. Quando o desenvolvedor criar um código driver (que invoca um kernel), este também deve receber o contexto como argumento.

O processo de inicialização compreende verificar quantos dispositivos (ndisp) existem na máquina e associar ao contexto o dispositivo dispNum MOD ndisp, onde dispNum é o número do dispositivo passado para a sub-rotina HLIB_CriaContexto. Em alguns backends como o OpenCL, também é necessário criar uma fila de execução padrão para as operações internas 
da biblioteca. Em CUDA, existem chamadas síncronas que não necessitam de uma fila (cudaStream). O comportamento dessa fila de execução padrão será descrito na Seção 3.2.6.

Internamente, o processo de inicialização é diferente para cada backend, a tabela 3.2 descreve as operações executadas em cada um:

\begin{tabular}{|l|c|c|c|}
\hline \multicolumn{1}{|c|}{ Backend } & CUDA & hStreams & OpenCL \\
\hline Operação & & hStreams_Init & clGetPlatformIDs \\
\hline Obter plataforma & cudaGetDeviceCount & hStreams_GetNumPhysDomains & clGetDeviceIDs \\
\hline Associar dispositivo & cudaSetDevice & hStreams_AddLogDomain & clCreateContext \\
\hline Criar fila padrão & & hStreams_StreamCreate & clCreateCommandQueue \\
\hline
\end{tabular}

Tabela 3.2: Inicialização interna da HLIB em diferentes arquiteturas.

Ao final da aplicação, o desenvolvedor deve destruir o contexto com o dispositivo utilizando a sub-rotina a seguir:

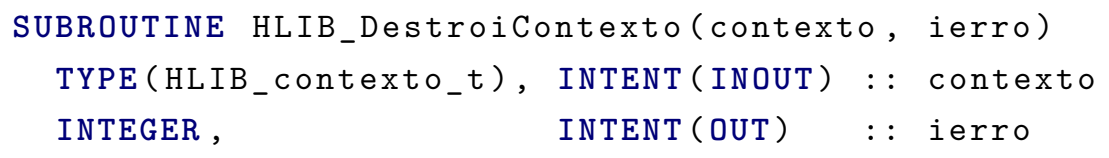

\subsection{2}

\section{Alocar / desalocar memória no dispositivo}

Os arrays necessários à execução de um kernel devem ser explicitamente alocados no dispositivo. As memórias da CPU e do dispositivo são distintas e normalmente possuem um desempenho diferente, conforme a Figura 3.1.

Na computação heterogênea existem dois modelos distintos de alocação de memória, alocação explícita e implícita. Por exemplo, o OpenACC e o OpenMP fazem a alocação de memória no dispositivo de forma implícita e em CUDA e OpenCL cabe ao desenvolvedor alocar explicitamente a memória no dispositivo. Adotamos a estratégia de alocação explícita: na HLIB o desenvolvedor explicitamente aloca a memória no dispositivo.

Para alocar memória em um dispositivo associado a um contexto, o programador deve invocar a sub-rotina a seguir:

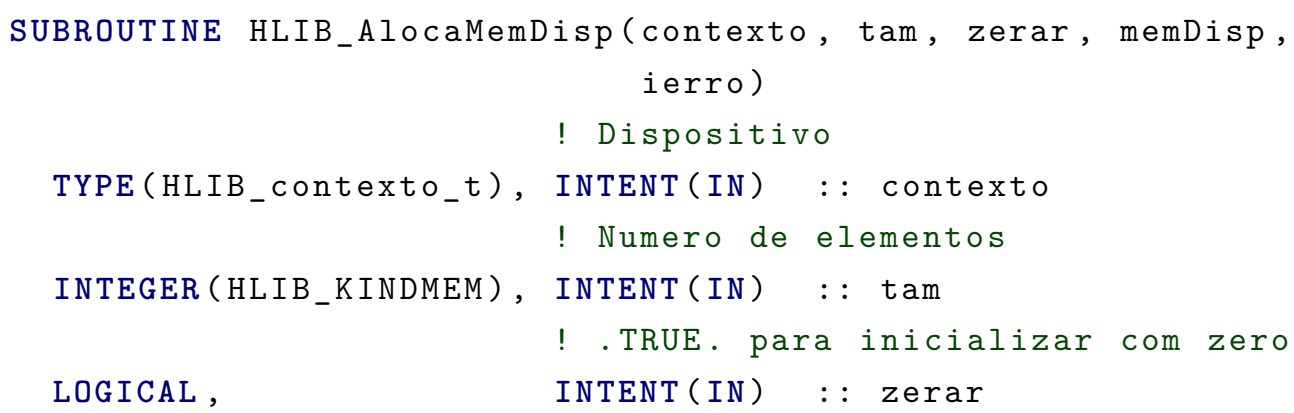




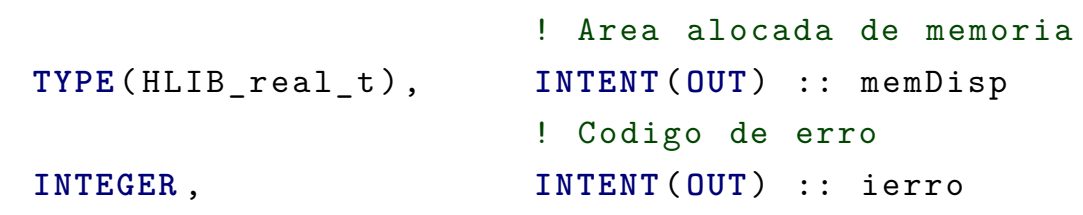

Essa sub-rotina aloca a área de memória no dispositivo e devolve um objeto opaco, como por exemplo o HLIB_real_t que mapeia para o tipo básico float no dispositivo. Como no OpenCL, a memória alocada no dispositivo é um objeto opaco, implementamos da mesma forma para manter a compatibilidade com o OpenCL.

Em CUDA e hStreams, a memória no dispositivo é um ponteiro como outro qualquer, aumentando a flexibilidade de programação, pois a aritmética de ponteiro e a indexação ocorrem da mesma forma que em $\mathrm{C} / \mathrm{C}++$. Mas para manter a interseção de APIs da Figura 3.5, definimos nossa API da mesma forma que a API menos flexível, neste caso OpenCL. Os backends CUDA, hStreams e regular armazenam o ponteiro para a memória do dispositivo dentro do objeto opaco.

A biblioteca armazena no objeto a quantidade de memória alocada, assim é possível verificar invasão de memória em operações de cópia. Definimos alguns tipos básicos para alocação de memória no dispositivo:

- HLIB_real_t

- HLIB_double_t

- HLIB_int32_t

- HLIB_int64_t

Com exceção do backend regular, em todos os outros a implementação é feita invocando a função correspondente para alocação de memória no dispositivo como cudaMalloc em CUDA. O backend regular invoca um simples malloc em C, pois só existe a memória principal.

A memória do dispositivo é desalocada com a sub-rotina a seguir:

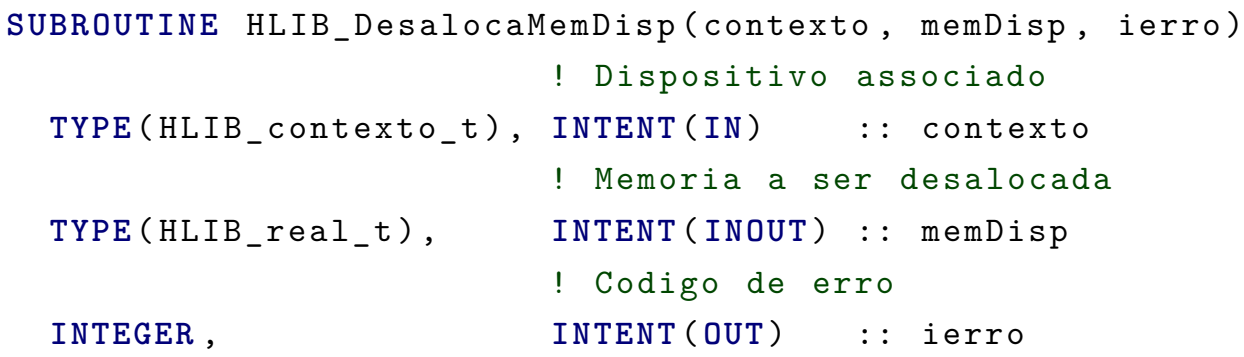

A biblioteca também verifica se o contexto foi criado e se a memória foi alocada antes de ser desalocada. 


\subsection{3}

\section{Memória para transferências entre CPU e dispositivo}

Na HLIB, as transferências entre CPU e dispositivo são explícitas, assim como em CUDA, OpenCL e hStreams. Por exemplo, o desenvolvedor pode inicializar um array na memória da CPU com dados lidos do disco e copiar seu conteúdo para um array equivalente na memória do dispositivo, e pode copiar um array alocado na memória do dispositivo, que é a saída de um kernel, para um array equivalente na memória da CPU. Esse padrão é bem comum na computação heterogênea.

Em arquiteturas onde o dispositivo é conectado via barramento PCI Express, conforme a Figura 3.1, é possível aumentar o desempenho entre cópias de memória $\mathrm{CPU} \Leftrightarrow$ dispositivo alocando uma memória pinned [65, 66, 67]. Esse tipo de memória é alocada na memória da CPU e não pode ser paginada (swap) pelo sistema operacional. Com isso é possível fazer operações do tipo DMA (direct memory access), habilitando a sobreposição das cópias de memória com execuções de kernels (em CUDA esta sobreposição é feita com as chamadas assíncronas, que só funcionam se a memória alocada na CPU for pinned[68]). O uso desse tipo de memória para transferências $\mathrm{CPU} \Leftrightarrow$ dispositivo é recomendado pois essas transferências ocorrem no barramento PCI Express, que é o gargalo[69] de muitas arquiteturas heterogêneas.

Para alocar a memória pinned localizada na memória da CPU, o desenvolvedor invoca a sub-rotina a seguir:

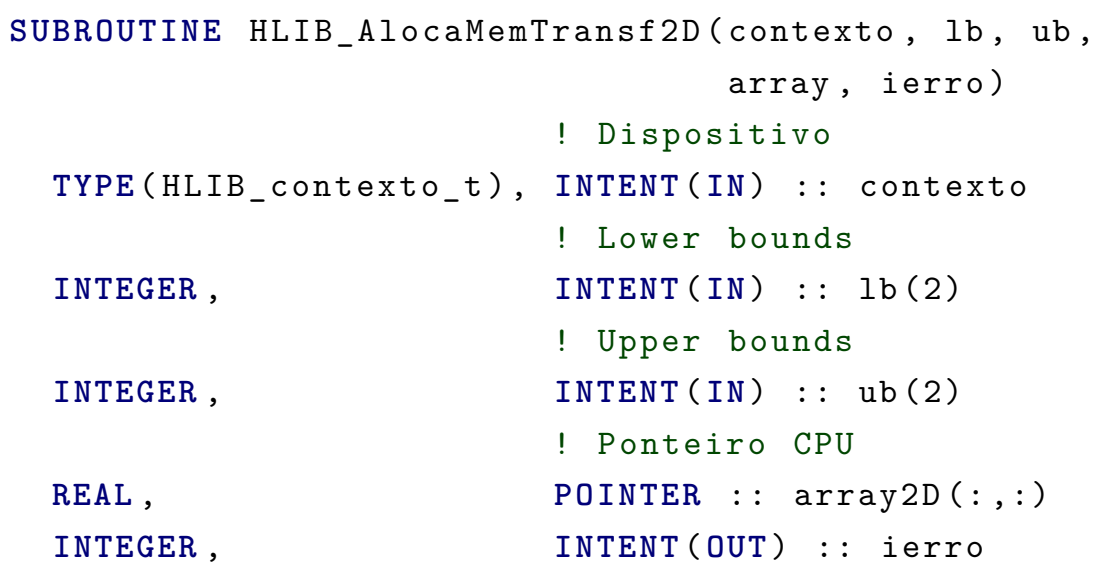

A função anterior aloca um array 2D de números reais em uma memória pinned, localizada na memória da CPU. A biblioteca também oferece as interfaces para arrays de números inteiros e de três dimensões. Esse tipo de alocação com lower e upper bounds é muito útil, aumentando a flexibilidade na indexação de arrays, que não precisam iniciar no índice 1. 
Essa memória pinned é acessada como qualquer outro array Fortran 90, dando flexibilidade ao desenvolvedor. No backend regular essa alocação é feita com um simples ALLOCATE.

\subsection{4}

\section{Zerar memória alocada no dispositivo}

Para inicializar com zero (todos bytes $\leftarrow$ 0x00) uma memória alocada no dispositivo, basta invocar a sub-rotina a seguir:

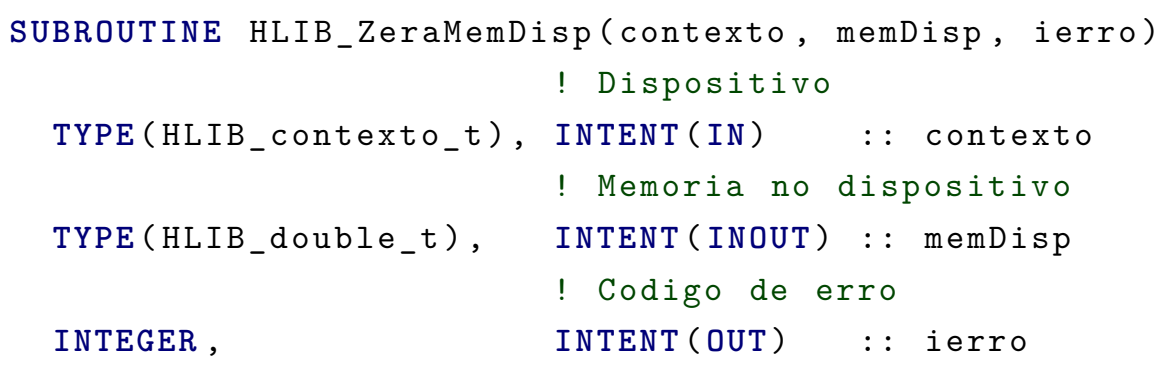

\section{2 .5}

\section{Cópia de memória $\mathrm{CPU} \Leftrightarrow$ dispositivo e dispositivo $\Leftrightarrow$ dispositivo}

$\mathrm{Na}$ HLIB as transferências de memória são explícitas. É possível fazer cópias entre as memórias da CPU e do dispositivo, e entre arrays alocados na memória do dispositivo.

Como a memória alocada no dispositivo é um objeto opaco, para se definir a posição inicial da cópia de memória é necessário passar um offset explícito para a sub-rotina de cópia. Em OpenCL o tipo opaco cl_mem é utilizado para representar a memória do dispostivio. Não é possível indexar diretamente este objeto como por exemplo ptr $[i d x]$ ou ptr+=idx, o desenvolvedor deve passar como argumento o offset idx.

Também é preciso passar explicitamente a direção da cópia que pode ser $\mathrm{CPU} \Rightarrow$ dispositivo ou dispositivo $\Rightarrow \mathrm{CPU}$. A sub-rotina de cópia entre as memórias da CPU e do dispositivo é mostrada a seguir:

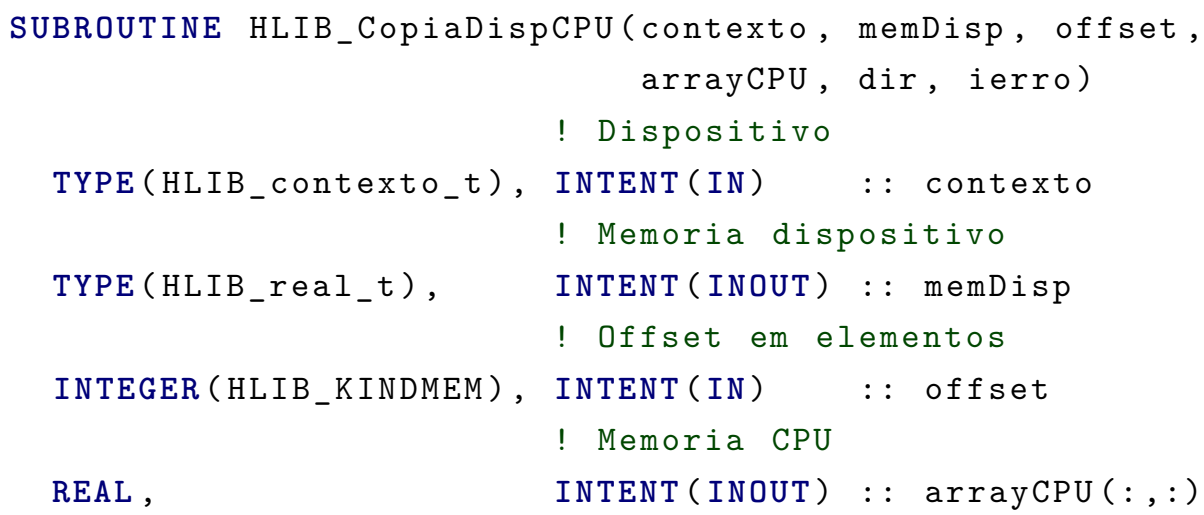


INTEGER,

INTEGER，
! Direcao da copia

INTENT(IN) : : dir

! Codigo de erro

INTENT(OUT) : : ierro

A interface acima define a cópia para arrays 2D de números reais. A biblioteca provê diversas interfaces Fortran 90 com verificação de tipo. Como existe um tipo básico associado à memória do dispositivo, a interface só permite a invocação de cópias de memória entre arrays do mesmo tipo, como um array na CPU tipo DOUBLE PRECISION e um array tipo HLIB_double_t no dispositivo.

O parâmetro offset indica a posição inicial da cópia no array da memória do dispositivo. Os arrays localizados na memória do dispositivo são organizados como um memória 1D linear, e o uso do offset tem a mesma semântica de indexação de um ponteiro $\mathrm{C} / \mathrm{C}++$, conforme o exemplo a seguir:

array_no_dispositivo[offset]

O próximo exemplo dispara uma cópia da memória do dispositivo para a memória da CPU, iniciando pelo centésimo elemento na memória do dispositivo (offset=99). A direção da cópia é determinada pelas constantes HLIB_DESTINO_CPU e HLIB_ORIGEM_CPU.

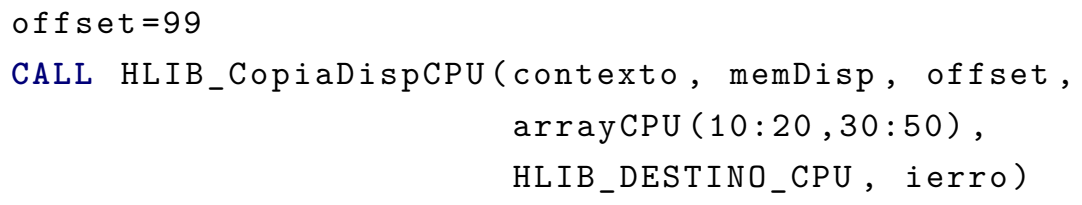

A quantidade de elementos da cópia é determinada pelos lower e upper bounds do arrayCPU localizado na memória da CPU. Antes da cópia, a HLIB verifica se haverá uma invasão de memória, pois tanto o tamanho do array do dispositivo, quanto o tamanho do array da CPU são conhecidos.

A sub-rotina para cópia entre memórias alocadas no dispositivo recebe dois arrays, um de origem e outro de destino, que devem ser do mesmo tipo básico. Para cada array um offset em elementos é informado. A seguir uma das interfaces para cópias de memória do dispositivo:

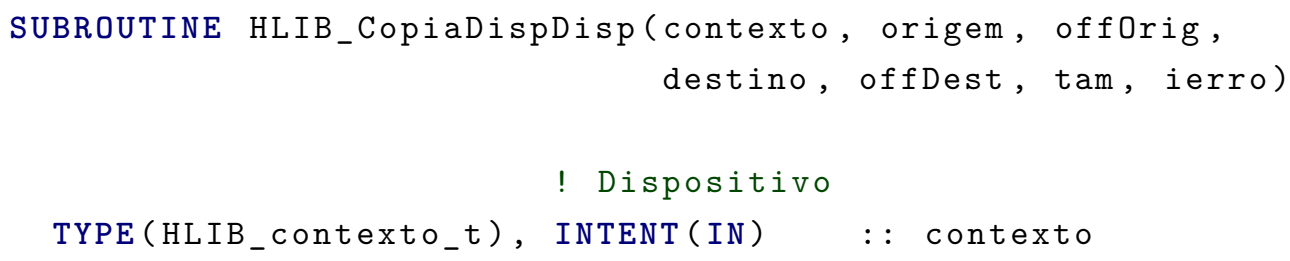




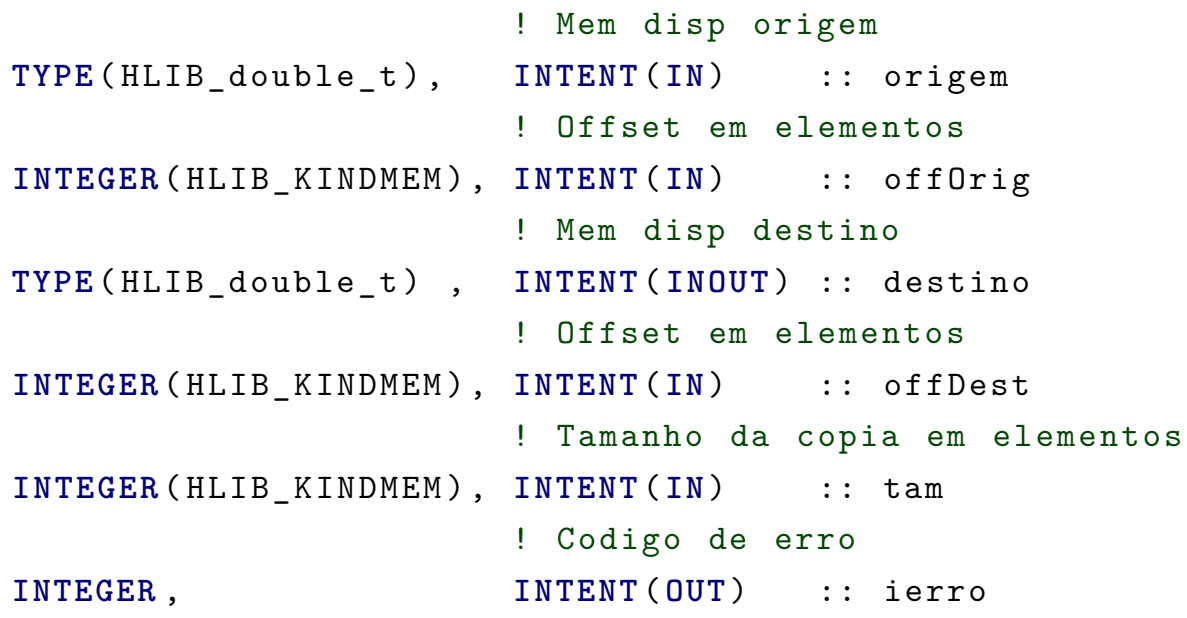

O argumento tam define o número de elementos a serem copiados. A HLIB também verifica se haverá uma invasão de memória.

\subsection{6}

\section{Filas de execução concorrentes}

A biblioteca também provê a capacidade de gerenciar filas de comandos a serem executados no dispositivo. Um comando pode ser uma transferência de memória ou uma execução de kernel. Cada fila é uma estrutura FIFO (first in first out). Dentro da mesma fila, um comando só pode começar a executar quando o anterior terminar por completo.

A funcionalidade de fila é implementada de forma diferente pelo OpenCL, CUDA e hStreams. Em uma fila cl_command_queue (OpenCL), dependendo da configuração, os comandos podem executar fora de ordem, enquanto que em uma fila cudaStream (CUDA), um comando só inicia após o encerramento do anterior, e em uma fila HSTR_LOG_DOM (hStreams), um comando pode iniciar se não houver dependência de dados com os comandos em execução naquela fila. O funcionamento das filas da HLIB é igual ao cudaStream da Nvidia, que é um caso particular do OpenCL e hStreams.

Os comandos em filas distintas podem executar em qualquer ordem e simultaneamente, i.e. filas distintas indicam operações independentes, que podem ser executadas de forma concorrente e fora de ordem. Por exemplo, uma cópia de memória $C_{1}$ inserida na fila $_{1}$ em um instante anterior ao da inserção de uma cópia $C_{2}$ na $f i l a_{2}$ poderá executar após $C_{2}$. Nada pode se afirmar sobre a ordem de execução de $C_{1}$ e $C_{2}$, pois são operações independentes.

A API da HLIB induz o desenvolvedor a implementar a concorrência de forma incremental, pois suas chamadas não recebem explicitamente a informação de qual fila utilizar. Acreditamos que o desenvolvedor deva primeiro desenvolver um código sequencial, e depois adicionar a concorrência de forma 
incremental, informando qual fila deverá ser utilizada antes de invocar uma chamada HLIB. Não expomos a primitiva enfileirar. Para enfileirar comandos em uma determinada fila, basta selecioná-la como sendo a fila corrente. Para enfileirar um kernel, o desenvolvedor deve utilizar a API específica no código do driver. A Listagem 3.1, apresenta um exemplo de um driver para a arquitetura CUDA. O driver do exemplo enfileira o kernel em uma fila do tipo cudaStream. Implementamos as seguintes primitivas de fila:

- Criar / destruir fila

- Selecionar fila

- Selecionar fila síncrona (padrão)

- Aguardar fila

- Testar se a fila está vazia

Após a seleção de uma fila, todos os comandos posteriores a seleção são enfileirados nessa fila. Os comandos são disparados em segundo plano, o controle volta para a CPU imediatamente. Para garantir que a execução de um comando terminou, é preciso aguardar a fila ou testar se ela está vazia. A seguir a rotina de criação de fila:

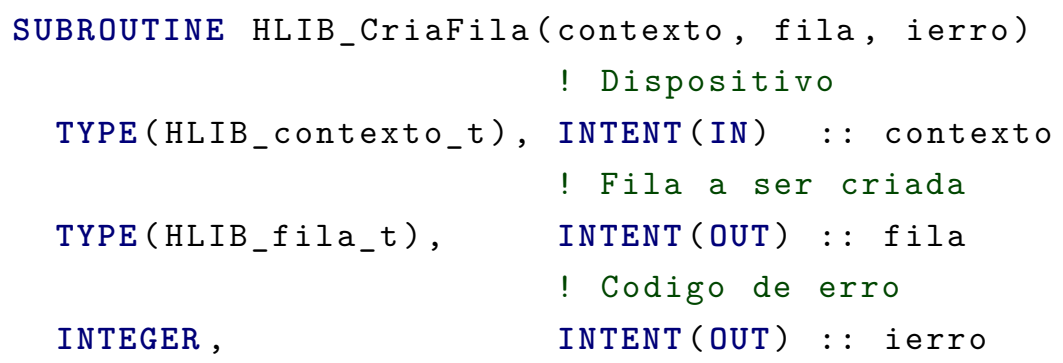

Todo contexto possui uma fila síncrona padrão, que é a fila corrente desde o momento da criação do contexto. Se o desenvolvedor não criar e selecionar fila alguma, a fila síncrona padrão será utilizada em todos os comandos e não é preciso testar se um comando acabou. Ou seja, o comportamento padrão da HLIB é sempre síncrono (fila síncrona padrão): cada comando só começa a executar após o termino do anterior, e toda chamada bloqueia a CPU até o seu término. Uma fila só pode estar associada a um único objeto contexto (um único dispositivo), que deve ser informado no momento da criação da fila.

Porém toda fila criada explicitamente é assíncrona em relação à CPU: se uma tal fila está selecionada, nenhuma chamada à HLIB bloqueia a CPU. Com isso também é possível obter concorrência entre a CPU e o dispositivo. Essa opção de seleção de filas deixa o desenvolvedor livre para começar a 
desenvolver com uma abordagem síncrona e adicionar a execução concorrente posteriormente, quando o código estiver mais maduro, pronto para receber otimizações. As sub-rotinas de cópia de memória não recebem a fila como argumento. O programador deve selecionar a fila com a sub-rotina de seleção de fila.

Os objetos HLIB_fila_t podem ser passados para os drivers criados pelo desenvolvedor. Estes objetos contêm a estrutura nativa da arquitetura em uso, como cudaStream em máquinas com GPUs Nvidia, conforme a Listagem 3.1.

As cópias do tipo $\mathrm{CPU} \Leftrightarrow$ dispositivo só poderão ser enfileiradas em uma fila assíncrona se a memória alocada na CPU for pinned. A rotina HLIB_AlocaMemTransf descrita anteriormente provê esse tipo de alocação.

As filas são muito utilizadas para sobrepor as cópias de memória com execução de kernels. Alguns dispositivos, como as GPUs atuais da Nvidia, suportam sobrepor duas cópias em sentidos diferentes e execução de kernel, permitindo esconder a latência da cópia de memória.

Em uma aplicação que implementa concorrência com filas, se o tempo de execução do kernel for maior que o tempo das cópias de memória, apenas o tempo do kernel irá contabilizar para o tempo total de execução. O tempo gasto com as cópias de memória não afeta o tempo de execução da aplicação. A seguir um exemplo da sobreposição de cópia de memória e execução de kernel:

Listagem 3.2: Pseudocódigo da sobreposição de cópia de memória e execução de kernel

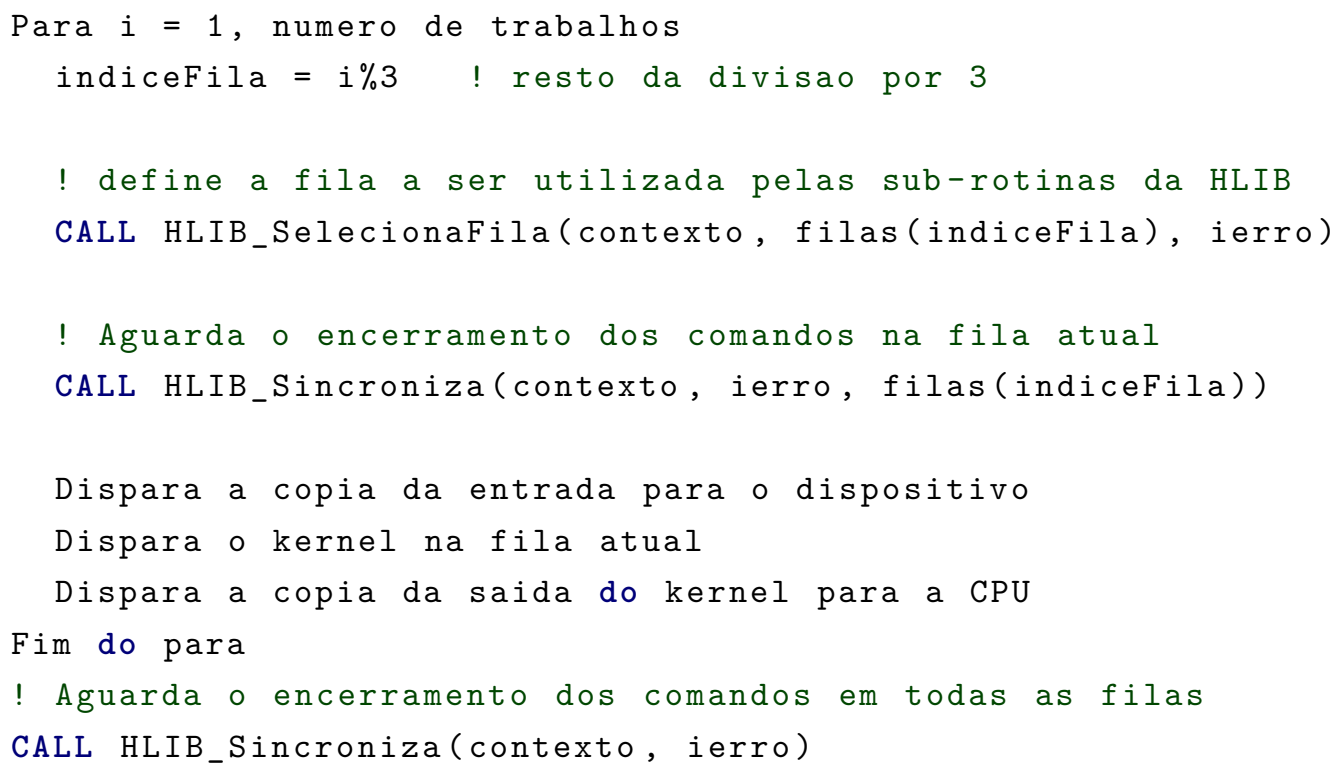

$\mathrm{Na}$ Listagem 3.2, enquanto o dispositivo executa o trabalho $_{i}$ o resultado do trabalho $_{i-1}$ é copiado de volta para a CPU e a entrada do trabalho $_{i+1}$ é copiada para o dispositivo. Como a fila tem um comportamento FIFO a cópia 
do resultado do trabalho $_{i}$ só inicia após a execução do kernel do trabalho que só inicia após a cópia da entrada do trabalho $_{i}$, pois todas essas operações são enfileiradas na mesma fila.

A sub-rotina HLIB_Sincroniza (primitiva aguardar fila) bloqueia a execução da CPU enquanto houver trabalho na fila. Se o desenvolvedor não informar a fila, a CPU fica bloqueada até todos os trabalhos do contexto ecenrrarem, conforme o exemplo da Listagem 3.2.

A Figura 3.6 mostra a execução no dispositivo utilizando as três filas. Note que se o tempo de cópia for menor ou igual ao tempo do kernel o dispositivo sempre estará executando um kernel. No caso da Figura 3.6b três comandos executam simultaneamente no dispositivo.

\begin{tabular}{|c|c|c|c|c|c|c|c|}
\hline Fila A & entrada1 & \multicolumn{2}{|c|}{ kernel1 } & resultado1 entrada4 & & kernel4 & resultado4 \\
\hline Fila B & & entrada2 & & kernel2 & resultado2 & & kerne15 \\
\hline Fila C & & & entrada3 & & kernel3 & resultado 3 & \\
\hline
\end{tabular}

(a)

\begin{tabular}{|c|c|c|c|c|c|c|c|c|c|}
\hline Fila A & entrada1 & kernel1 & resultado1 & entrada4 & kernel4 & resultado4 & entrada7 & kernel7 & resultado7 \\
\hline Fila B & & entrada2 & kernel2 & resultado2 & entrada5 & kernel5 & resultado5 & & \\
\hline Fila C & & & entrada3 & kernel3 & resultado3 & entrada6 & kernel6 & resultado6 & \\
\hline
\end{tabular}

(b)

Figura 3.6: Uso do dispositivo ao longo do tempo. Execuções de kernel em azul, cópias de memória em verde e nada sendo executado em vermelho. (a) um exemplo de execução onde o tempo de cópia é menor que o tempo do kernel. (b) um exemplo de execução onde o tempo de cópia é igual ao tempo do kernel.

Essa sobreposição de cópia de memória com execução de kernel tem um potencial de ganho de desempenho de até 3 vezes, caso onde o tempo de cópia de ida e o tempo de cópia de volta são iguais ao tempo do kernel, pois essas três operações vão ocorrer em paralelo, conforme Figura 3.6b.

A Figura 3.7 utiliza o exemplo da Figura 3.6a mostrando o comportamento das três unidades de execução do dispositivo: execução de kernel, cópia para o dispositivo e cópia do dispositivo.

\begin{tabular}{|l|l|l|l|l|l|l|l|l|l|l|}
\hline Execução & & \multicolumn{2}{|c|}{ kernel1 } & \multicolumn{2}{c|}{ kerne12 } & \multicolumn{2}{c|}{ kernel3 } & \multicolumn{2}{c|}{ kernel4 } & \multicolumn{2}{c|}{ kerne15 } \\
\hline CPU->Disp & entrada1 & entrada2 & entrada3 & & entrada4 & & entrada5 & & entrada6 & entrada7 \\
\hline Disp->CPU
\end{tabular}

Figura 3.7: Uso das unidades de execução do dispositivo ao longo do tempo. Execuções de kernel em azul, cópias de memória em verde e nada sendo executado em vermelho.

Podemos notar na Figura 3.7 que a unidade de execução de kernel fica sempre cheia após a cópia da entrada do primeiro kernel. É possível afirmar que o tempo de execução amortizado, será o maior valor entre os tempos de cópia e de kernel, quando é possível executar as três operações em paralelo. 


\subsection{7}

\section{Passagem de Mensagem entre Dispositivos}

A primitiva de troca de mensagens entre dispositivos não faz parte de nenhuma API da Figura 3.5. Em CUDA, é possível acessar a memória de outra GPU, mas ela deve estar instalada na mesma máquina. Implementamos chamadas equivalentes às do MPI para troca de mensagens entre dispositivos, utilizando o prefixo HLIB_MPI. Essas chamadas aceitam buffers alocados nos dispositivos. Nossa implementação, em sua camada mais baixa, invoca o MPI. Somente utilizamos funções do padrão MPI 1.1[70], com isso nossa implementação deverá funcionar com qualquer implementação da biblioteca MPI. Implementamos apenas algumas chamadas HLIB_MPI, conforme a demanda das aplicações do Capítulo 4.

Nos backends onde a memória do dispositivo é diferente da memória da $\mathrm{CPU}$, existe um passo interno adicional para copiar a memória alocada no dispositivo para um buffer intermediário na memória da CPU, então o buffer da CPU é passado para a chamada MPI equivalente. Todo esse processo é transparente para o usuário.

A Figura 3.8 mostra a troca de mensagens entre dispositivos em um processo MPI trocando informações com dois vizinhos, um à esquerda e outro à direita. A biblioteca quebra a mensagem enviada em pedaços para sobrepor a comunicação MPI com as cópias de memória e execuções de kernel. Podemos notar que o tempo de comunicação não aumenta o tempo total de execução (em azul) e até cinco operações ocorrem simultaneamente na Figura 3.8:

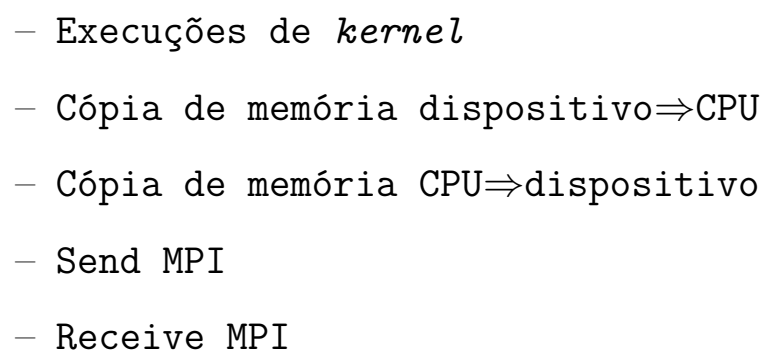

O primeiro HLIB_MPI_sendRecv da Figura 3.8 envia para a esquerda e recebe da direita. O primeiro pedaço da esquerda $\left(e_{1}\right)$ é copiado do dispositivo para CPU, então $e_{1}$ é enviado para o vizinho da esquerda e o primeiro pedaço do vizinho da direita $\left(v d_{1}\right)$ é recebido com MPI_sendRecv $\left(e_{1}, v d_{1}\right)$, então $v d_{1}$ é copiado para o dispositivo, repetindo esses passos até todos os pedaços acabarem. Então o segundo HLIB_MPI_sendRecv, que envia para a direita e recebe da esquerda é iniciado, com o primeiro pedaço da direita $\left(d_{1}\right)$ sendo copiado do dispositivo para CPU e assim por diante. 


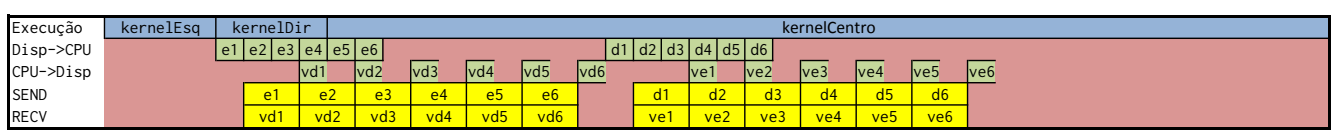

Figura 3.8: Comportamento de dois HLIB_MPI_sendRecv para trocar as bordas com dois processos MPI vizinhos. Eixo do tempo na horizontal. Execuções de kernel em azul, cópias de memória em verde, comunicação MPI em amarelo e nada sendo executado em vermelho. Mensagem quebrada em 6 pedaços. $e_{1}=$ pedaço $o_{1}$ da esquerda do processo corrente, ev $v_{1}=$ pedaço $o_{1}$ do vizinho da esquerda, $d_{1}=$ pedaço $o_{1}$ da direita do processo corrente, $v d_{1}=$ pedaço $o_{1}$ do vizinho da direita.

Para melhor visualização, na Figura 3.8 definimos a velocidade das cópias $\mathrm{CPU} \Leftrightarrow$ dispositivo como duas vezes a velocidade da rede, ficando próximo do observado na prática com redes Infiniband[71].

Na Figura 3.8 cinco filas são envolvidas, utilizamos uma fila para cada invocação de kernel e a sub-rotina HLIB_MPI_SendRecv utiliza uma fila interna para cópias de memória dispositivo $\Rightarrow \mathrm{CPU}$ e outra fila interna para cópias de memória $\mathrm{CPU} \Rightarrow$ dispositivo.

O backend regular (computação homogênea) invoca o MPI diretamente, pois só existe a memória principal, e neste caso a memória do dispositivo é alocada na memória principal.

Até recentemente não existia suporte MPI à comunicação direta entre GPUs CUDA[72]. Nosso backend CUDA implementa essa comunicação sem depender de tal suporte, usando as funções CUDA IPC Inter-Process Communications. Atualmente existem implementações CUDA-aware MPI[73, 74]. Estas implementações aceitam, de forma transparente, buffers alocados na GPU. A última versão do nosso backend CUDA apenas invoca o CUDA-aware MPI $[73,74]$ se o mesmo estiver disponível. Senão, ele utiliza o mesmo código dos outros backends heterogêneos. Definimos esta funcionalidade em tempo de compilação.

\section{3}

\section{Avaliação}

As aplicações do Capítulo 4, RTM e FWI da Petrobras, utilizam a HLIB com as arquiteturas CUDA e convencional (somente CPU). Com a aplicação RTM, realizamos uma prova de conceito utilizando a HLIB (backend hStreams) com placas Xeon Phi KNC.

Executamos a aplicação RTM com sucesso nas plataformas - CPU Power8 + GPU Nvidia, CPU Intel x86 + GPU Nvidia e apenas CPU Intel x86 utilizando os compiladores Intel e IBM XL para a CPU e o nvcc para a GPU. 
Os testes compreendem as versões do CUDA: 4, 5, 6.5 e 7.5.

Testamos nossas funções de troca de mensagens entre dispositivos com com diversas implementações MPI - OpenMPI, Intel, IBM e Cray - e diversos tipos de rede - Ethernet 10Gb, Infiniband e Cray.

Não tivemos que alterar nenhuma linha de código da HLIB para executar em todas essas plataformas.

Fizemos uma avaliação informal da HLIB em conjunto com um desenvolvedor usuário da HLIB. Constatamos alguns benefícios ao utilizar a HLIB:

- Curva de aprendizado rápida

- Alterar na biblioteca, resolve todos os projetos que a utilizam

- Encapsular boas práticas, como a verificação de invasão de memória da Seção 3.2 .5

- Facilitar a portabilidade para outros paradigmas ex:OpenCL, mas é necessário reescrever o kernel.

- Suporte à troca de mensagens entre dispositivos (MPI)

- Apesar da Nvidia alegar que CUDA tem retrocompatibilidade, sabemos que não é sempre assim (a HLIB trata internamente diferentes versões de CUDA)

O desenvolvedor citou esse problema da retrocompatibilidade CUDA porque recentemente um outro desenvolvedor, que está codificando diretamente em CUDA, passou pelo mesmo problema que já havíamos solucionado na HLIB. Esse problema se refere a alocação de memória não paginável (Seção 3.2.3), e é difícil de encontrar. O código compila mas a aplicação aborta com um runtime error, que não informa a origem do erro.

\section{4}

\section{Discussão}

O uso da HLIB induz o desenvolvedor a dividir a aplicação em duas camadas, basta ele utilizar a mesma interface para definir o código driver que enfileira o kernel no dispositivo. Com isso, a camada agnóstica de arquitetura enfileira o kernel com uma única chamada para todas as arquiteturas.

A HLIB mantém a mesma abordagem das APIs CUDA, OpenCL e hStreams. Com a HLIB, o desenvolvedor utiliza o mesmos paradigmas dessas APIs. Cabe ao desenvolvedor gerenciar a memória do dispositivo e enfileirar execuções de kernels e as transferências entre a memória da CPU e a memória do dispositivo. 
Se uma aplicação tira benefício de uma funcionalidade específica de uma arquitetura, então haverá uma perda de desempenho ao se utilizar a HLIB. Mas caso contrário, a perda de desempenho é apenas referente a uma invocação Fortran. A biblioteca se comporta como um wrapper Fortran 90 para as APIs específicas de cada arquitetura.

Poderemos no futuro, adicionar à HLIB extensões com funcionalidades específicas de cada arquitetura. Caso o desenvolvedor opte pelo uso das extensões, haverá uma perda de portabilidade na camada superior. Pois essa camada passa a tratar diferentes arquiteturas. O desenvolvedor também pode estender a HLIB com funcionalidades específicas.

Como cada dispositivo possui uma memória, assim como a CPU, escolhemos utilizar o MPI para agregar o poder computacional de múltiplos dispositivos, tendo em vista que o MPI já é utilizado em aplicações de memória distribuída. Nas aplicações do Capítulo 4, utilizamos um processo MPI por dispositivo. Com uma operação MPI_Comm_split encontramos o rank MPI dentro do nó, e com este rank, definimos o dispositivo a ser associado ao objeto contexto de cada processo MPI. 


\section{4}

\section{Aplicações de Alto Desempenho}

Neste capítulo, apresentamos as aplicações de imageamento sísmico Migração Reversa no Tempo RTM (Reverse Time Migration) e a Inversão Completa do Campo de Onda FWI (Full Waveform Inversion), que implementamos com a estratégia de duas camadas e as ferramentas HLIB e OpenVec.

\section{1}

\section{Migração Reversa no Tempo RTM}

A migração reversa no tempo[5] RTM (Reverse Time Migration), tem como finalidade gerar uma imagem de subsuperfície. De forma simplificada, podemos compará-la a um exame de ultrassom. O aparelho de ultrassom emite ondas que são refletidas pelos diferentes tecidos orgânicos, e baseandose no tempo de retorno de cada onda, posiciona cada reflexão gerando uma imagem. A migração RTM posiciona os eventos geológicos de forma mais correta que outras migrações que demandam menos poder computacional, como a migração Kirchhoff[75, 76]. Uma única execução da migração RTM pode ocupar uma máquina de um petaflop por três meses.

Um levantamento sísmico[77] pode ser visto na Figura 4.1. Este processo compreende milhares de experimentos, chamados de tiros (seismic shots), que registram nos sensores (receptores) as transições entre diferentes camadas geológicas. O levantamento sísmico da Figura 4.1 é realizado com o navio em movimento, ele se movimenta com velocidade constante ativando a fonte e registrando nos sensores a energia refletida pelas diferentes camadas geológicas. Cada registro de uma ativação da fonte representa um tiro e um conjunto de tiros compõe um dado sísmico. Quando posicionamos estes sensores em terra, os chamamos de geofones e quando os posicionamos no mar, os chamamos de hidrofones.

Um tiro é o processo onde uma fonte emite energia, e diversos sensores registram por $T$ segundos essa energia que é propagada e refletida pela terra. Quando posicionada em terra, podemos implementar a fonte com uma detonação de uma carga de TNT, normalmente enterrada a alguns metros de profundidade. Nos levantamentos sísmicos marítimos, um canhão de ar comprimido acoplado ao fundo do casco do navio gera a energia da fonte. O contraste entre as diferentes camadas geológicas reflete parte dessa energia, e os sensores registram a porção da energia que retorna a superfície.

Antes de executar a RTM, os profissionais da equipe de processamento sísmico estimam o modelo de velocidades, que de forma aproximada, repre- 


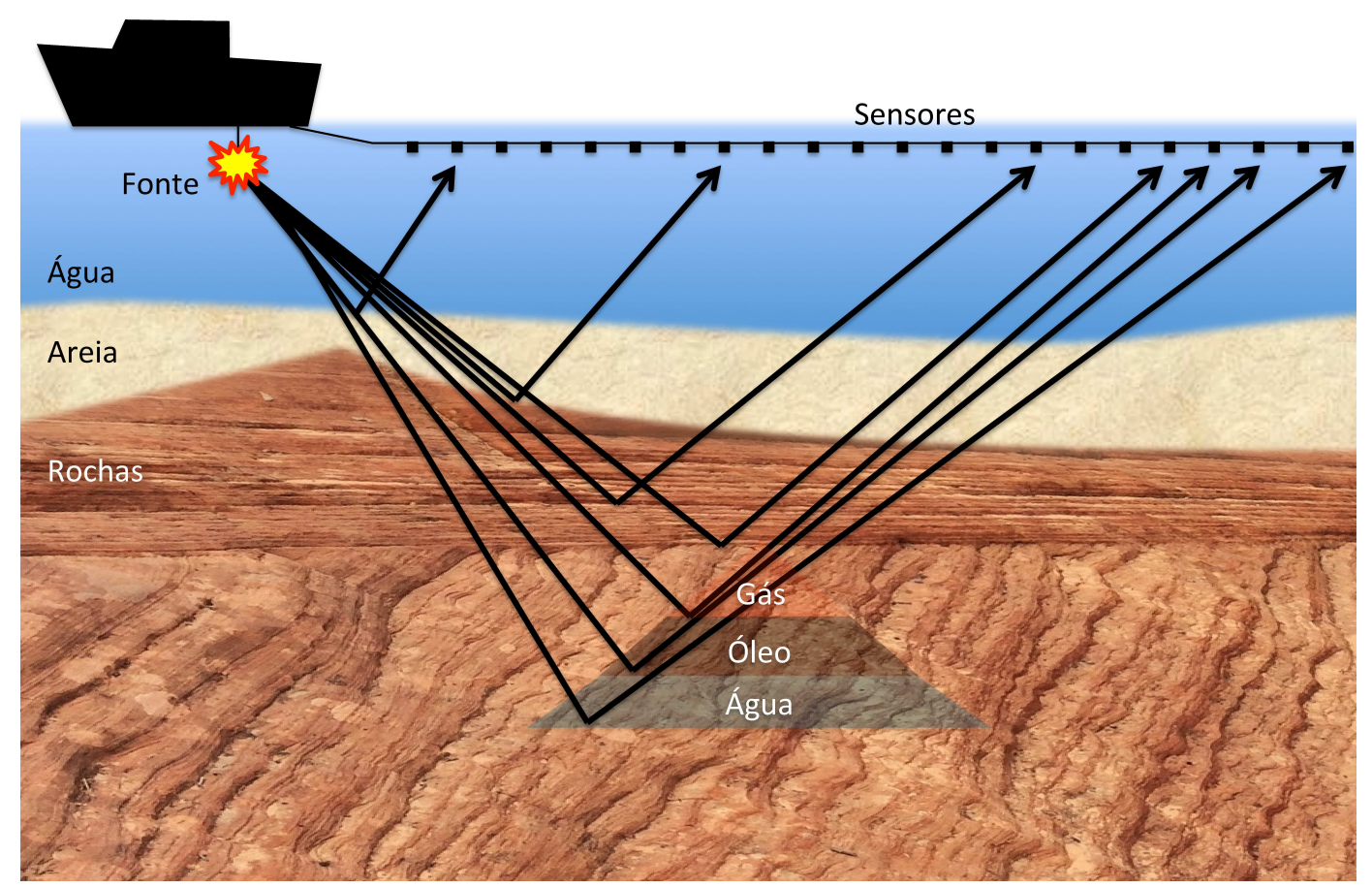

Figura 4.1: Levantamento sísmico marítimo. O navio puxa cabos com os sensores (hidrofones). Abaixo do casco do navio temos a fonte sísmica que é um canhão de ar comprimido. Parte da energia que a fonte produz é refletida pelas camadas geológicas do fundo do mar.

senta as velocidades de propagação da onda para as camadas geológicas do levantamento sísmico.

A migração RTM simula o caminho percorrido pela onda sísmica, que se inicia na posição da fonte e propaga em um volume ao redor do tiro. E também simula o caminho reverso da onda, que se inicia nas posições dos receptores (sensores) e utiliza os sinais registrados pelo levantamento sísmico. Ambas as simulações utilizam a Equação 4-1 para calcular a propagação da onda. A propagação leva em conta as velocidades estimadas do modelo de velocidade. A velocidade de propagação é o termo $c$ da Equação 4-1.

$$
\frac{1}{c^{2}} \frac{\partial^{2} p}{\partial t^{2}}=\left(\frac{\partial^{2} p}{\partial x^{2}}+\frac{\partial^{2} p}{\partial y^{2}}+\frac{\partial^{2} p}{\partial z^{2}}\right)
$$

Obtemos a posição do evento onde as ondas dos receptores e da fonte se encontram no mesmo passo de tempo (time step). Ou seja, a correlação das duas simulações (fonte e receptores) gera uma imagem 3D, conforme a Equação 4-2, onde $S$ é o campo da simulação da fonte (source), $R$ é o campo da simulação dos receptores e $T$ é o tempo total de registro do tiro. De forma simplificada a migração RTM simula o caminho percorrido pelo sinal para encontrar a posição onde ocorreu a reflexão que originou o sinal. A imagem 
final, resultado da migração, é a soma das imagens de todos os tiros, que podem ser processados de forma embaraçosamente paralela.

$$
\operatorname{Img}(x, y, z)=\sum_{t=0}^{T} S(x, y, z, t) \times R(x, y, z, t)
$$

O código de propagação de onda que representa mais de $95 \%$ do tempo de execução, utiliza o método de diferenças finitas para simular o caminho percorrido pela onda. O cálculo do próximo instante na simulação utiliza o tempo atual e o anterior (derivada temporal de segunda ordem), e vários pontos vizinhos do tempo atual para calcular as derivadas espaciais x,y,z da Equação 4-1. O cálculo das derivadas espaciais utiliza um estêncil como o da Figura 4.2. Escolhemos este tamanho de estêncil para efetuarmos uma comparação direta de desempenho com o estado da arte[22].

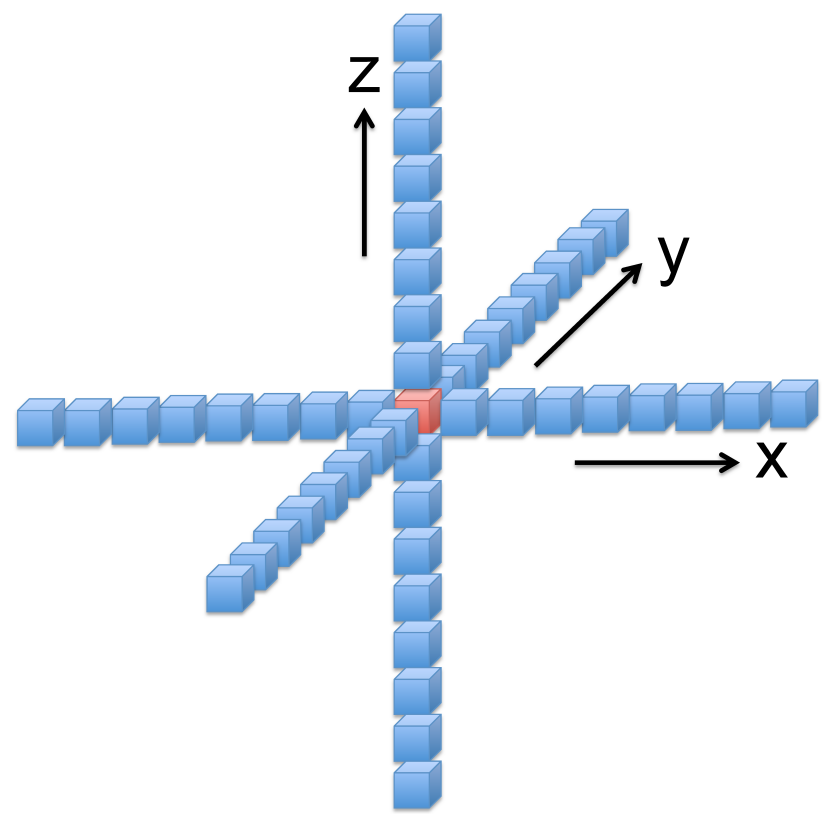

Figura 4.2: Estêncil para calcular derivadas espaciais de $16^{\mathrm{a}}$ ordem. O cálculo da derivada no ponto central (em vermelho) depende dos pontos vizinhos (em azul).

Nos nossos testes, utilizamos o mesmo tamanho de cela para as três dimensões. Esta decisão deixa os pesos iguais para as três dimensões. Os pesos variam apenas em função da distância ao ponto central. Com isso, o cálculo de cada ponto central da Figura 4.2 requer 62 operações de ponto flutuante (conforme a Listagem 4.2 com RAIO=8). Com essa demanda de operações, temos que a migração reversa no tempo em um levantamento sísmico de 400.000 tiros requer 736 exa $\left(10^{18}\right)$ operações de ponto flutuante, conforme a Tabela 4.1. 


\begin{tabular}{|l|r|}
\hline Número de Tiros & 400000 \\
\hline Simulações por Tiro & 2 \\
\hline Comprimento & 1500 \\
\hline Largura & 1100 \\
\hline Profundidade & 750 \\
\hline Iterações (passos de tempo) & 12000 \\
\hline Operações por Ponto & 62 \\
\hline Total de Operações & $736 \times 10^{18}$ \\
\hline
\end{tabular}

Tabela 4.1: Estimativa da quantidade de operações de ponto flutuante para uma migração RTM. O total de operações é o produto das demais linhas da tabela. As dimensões comprimento, largura e profundidade compreendem um volume discretizado em uma grade regular 3D.

A estimativa da Tabela 4.1 leva em conta apenas a solução da Equação 4-1. Para cada tiro executamos duas simulações. Uma para o campo de onda da fonte, e outra para o campo de onda dos receptores.

\section{2}

\section{Inversão Completa do Campo de Onda FWI}

A Inversão Completa do Campo de Onda (Full Waveform Inversion) FWI $[78,79,80,81]$ gera o modelo de velocidade a partir dos dados coletados. Com esse modelo é possível fazer uma migração RTM mais precisa, pois esse modelo de velocidade é mais realista.

Chamamos de traço sísmico o sinal registrado por um receptor de um tiro, e associamos a um traço um único par $\{$ tiro, receptor $\}$. Por exemplo, se o navio da Figura 4.1 puxa 1000 sensores podemos afirmar que cada tiro possui 1000 traços sísmicos.

O método FWI faz uma modelagem do traço sísmico de cada tiro e o compara com o respectivo traço registrado pelo levantamento sísmico. Essa comparação gera uma função de erro que é minimizada por um processo de otimização iterativo. Quanto mais próximo da realidade o modelo de velocidade estiver, menor é o erro entre o sinal modelado e o registrado.

Cada iteração desse processo faz uma migração RTM com o resíduo da comparação entre os traços modelados e registrados. O resultado é um gradiente que o método FWI utiliza como guia para gerar um novo modelo de velocidade a partir do modelo atual. Então o método aplica esse gradiente com vários pesos e faz uma busca linear para encontrar o melhor peso, gerando um novo modelo de velocidade que será utilizado pela próxima iteração. A Figura 4.3 apresenta os passos do método FWI. Como muitas iterações são necessárias para gerar um modelo de velocidade, o método FWI tem um custo computacional uma ordem de grandeza maior que a RTM. 


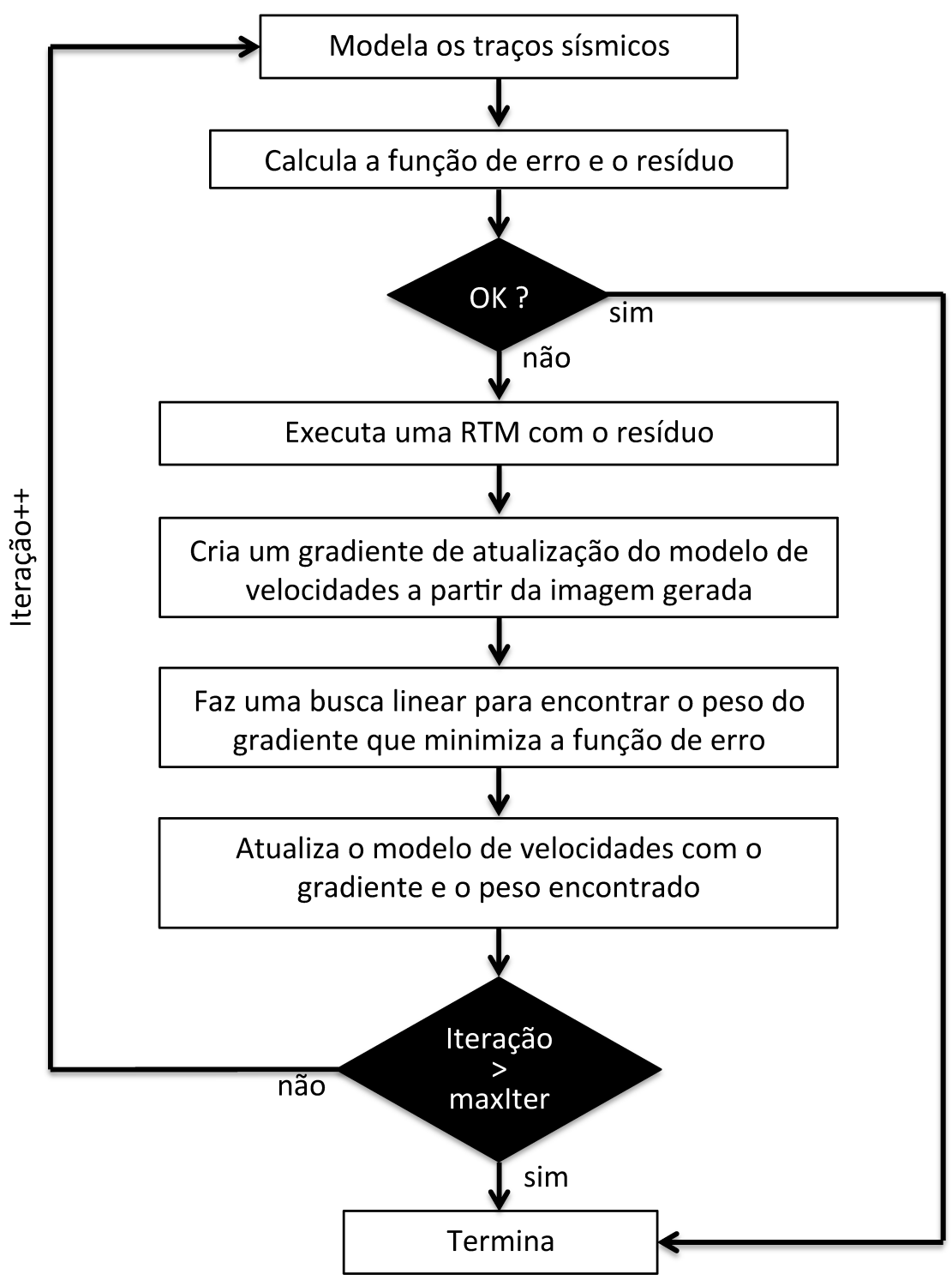

Figura 4.3: Fluxograma do método FWI.

\section{3}

\section{Observações}

As aplicações RTM e FWI demandam muito poder computacional. Uma execução da RTM pode ocupar uma máquina ${ }^{1}$ petaflop por três meses. Como a FWI executa uma RTM e várias modelagens a cada iteração, sua demanda computacional é ainda maior.

O tempo de execução destas aplicações depende muito da parametrização do usuário e dos dados de entrada. Por exemplo, o tempo de execução é diretamente proporcional ao número de tiros, profundidade do modelo e tempo de registro. Com relação à parametrização do usuário, o parâmetro que mais afeta o tempo de execução é a frequência máxima, que define a maior

${ }^{1}$ Máquina com 2,6 petaflops de pico teórico em precisão simples com 300 GPUs Nvidia K80 e rede Infiniband FDR. 
frequência presente na simulação. Quanto maior a frequência, maior o nível de detalhamento na imagem. O tempo de execução é proporcional a freqmax ${ }^{4}$, pois para contemplarmos uma determinada frequência máxima precisamos respeitar os critérios de estabilidade e dispersão numérica do método de diferenças finitas, que dependem de quatro parâmetros: $d x, d y, d z$ e $d t$, onde $d x, d y$ e $d z$ se referem ao tamanho da cela da grade 3D discretizada e $d t$ se refere ao passo de tempo.

Para executar a FWI no mesmo parque computacional que executa a RTM podemos reduzir o conteúdo de frequência. Por exemplo, uma redução de 50\% na frequência máxima, reduz o tempo de execução da simulação da propagação da onda em 16 vezes $\left(\frac{1}{2}\right)^{4}$. Normalmente a execução da FWI começa com um conteúdo de frequência baixo e aumenta a frequência máxima gradativamente com as iterações[82] do fluxo da Figura 4.3. Com isso, temos que as primeiras iterações do método FWI são menos custosas computacionalmente em relação a RTM pois utilizam frequências baixas como $3,5 \mathrm{~Hz}$ e $7,2 \mathrm{~Hz}$ de acrodo com o trabalho de Virieux e Operto[83].

\section{4}

\section{Exemplo de Uso HLIB}

Nesta seção, demonstramos o uso da HLIB em aplicações de imageamento sísmico. Estas aplicações demandam mais de um petaflop de capacidade de processamento. E podem utilizar mais de mil dispositivos em paralelo.

Em um ambiente paralelo, cada dispositivo recebe um trecho do volume sísmico. Nas aplicações RTM e FWI implementamos um kernel que simula a propagação do campo de ondas. Para calcular as derivadas espaciais, os pontos vizinhos são necessários, conforme a Figura 4.2.

Utilizamos as filas para sobrepor a computação com o envio (MPI) dos pontos necessários aos outros dispositivos. A Figura 4.4 mostra uma decomposição de domínio em uma dimensão com um processo trocando os pontos com seus vizinhos, satisfazendo as dependências do estêncil da Figura 4.2 .

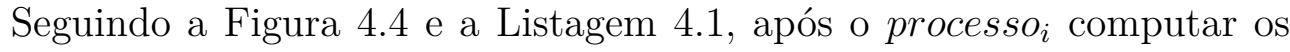
pontos da borda (em verde claro), o dispositivo inicia a computação dos pontos do centro (em verde escuro), ao mesmo tempo em que a troca as bordas com seus dois vizinhos, utilizando duas chamadas HLIB_MPI_sendRecv, conforme a Figura 3.8.

Fizemos a decomposição de domínio com MPI, utilizando um dispositivo por rank MPI. A seguir o pseudocódigo do propagador de ondas paralelo com troca de bordas: 


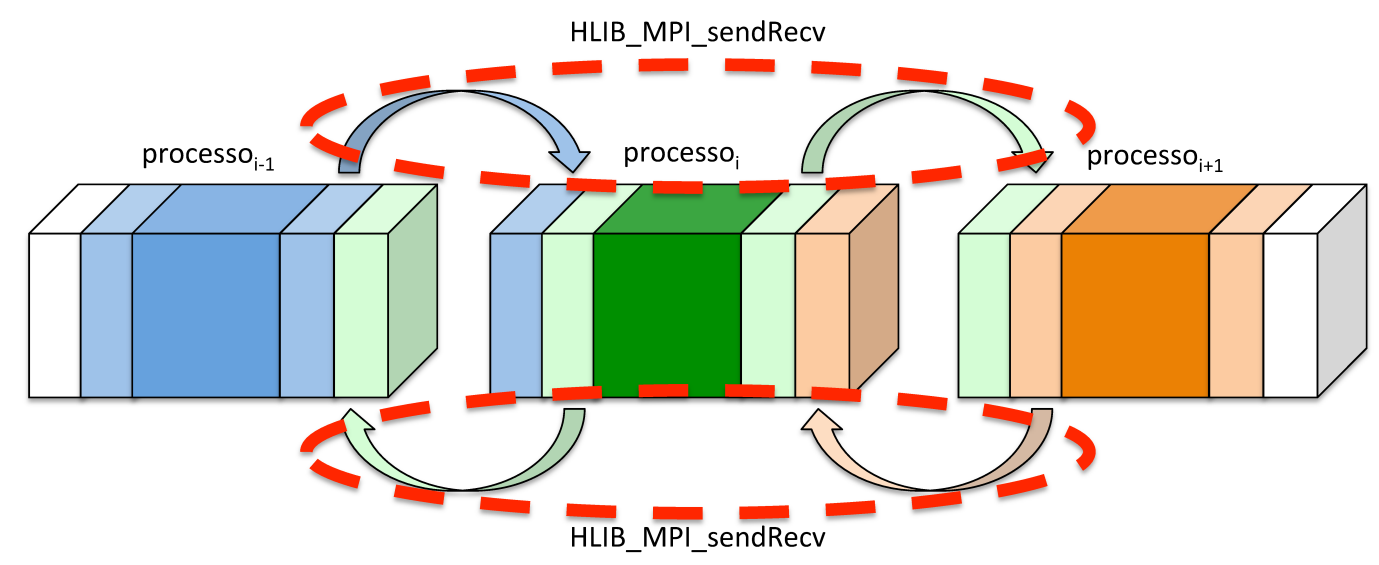

Figura 4.4: Troca de bordas do algoritmo de propagação de onda 3D por diferenças finitas, que é utilizado pelas aplicações RTM e FWI. Em verde

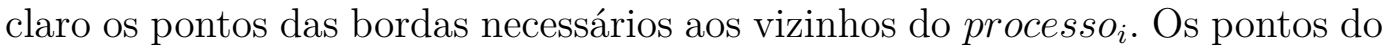
centro que não são dependência para nenhum outro processo, em verde escuro. O processo $i$ invoca HLIB_MPI_sendRecv duas vezes, conforme as setas.

Listagem 4.1: Pseudocódigo RTM/FWI para uma propagação de onda.

Como o tempo de comunicação é menor que o tempo de computação, podemos afirmar que o tempo de comunicação não afeta o tempo total de execução. No kernel CUDA de propagação de onda, obtivemos 751 GFlops/s por placa Nvidia K80 (duas GPUs). 
Utilizamos a o suporte da HLIB para troca de mensagens em um cluster com 2,6 petaflops de pico teórico com GPUs Nvidia K80 e rede Infiniband FDR capaz de transmitir dados a $56 \mathrm{~Gb} / \mathrm{s}$.

\section{5}

\section{Exemplo de Uso OpenVec}

As técnicas modernas de imageamento sísmico como a RTM e FWI utilizam o método de diferenças finitas (DF) para simular a propagação de um campo de onda em um volume geológico. Obter um ganho de desempenho nessas aplicações, traz um grande benefício, pois essas etapas podem consumir meses no fluxo de processamento sísmico utilizando clusters que custam dezenas de milhões de reais. Estas aplicações utilizam apenas operações de precisão simples (32 bits).

Os estêncis para o cálculo de DF aparecem em métodos iterativos para resolver equações diferenciais parciais, como a Equação 4-3.

$$
\frac{1}{c^{2}} \frac{\partial^{2} p}{\partial t^{2}}=\left(\frac{\partial^{2} p}{\partial x^{2}}+\frac{\partial^{2} p}{\partial y^{2}}+\frac{\partial^{2} p}{\partial z^{2}}\right)
$$

Para um ponto qualquer em uma grade regular, a computação estêncil é uma soma ponderada com pesos fixos em um conjunto de pontos. A Figura 4.5a mostra os pontos necessários ao cálculo de uma derivada espacial de $8^{\mathrm{a}}$ ordem para o ponto central (em vermelho). O cálculo da derivada temporal requer o ponto central do passo de tempo anterior e o ponto central do passo de tempo atual.

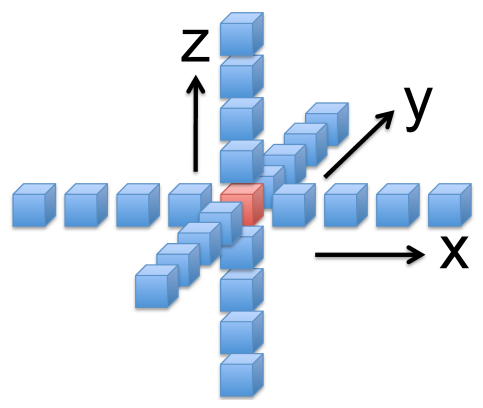

(a)

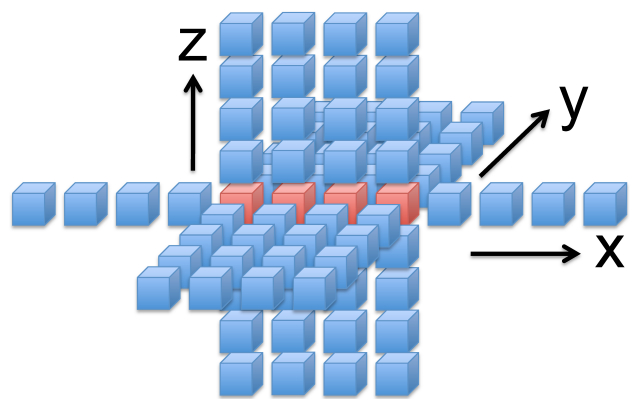

(b)

Figura 4.5: (a) um estêncil de 25 pontos $(\mathrm{RAIO}=4)$, obtemos o ponto central em vermelho com uma soma ponderada de todos os pontos. (b) 4 estêncis computados simultaneamente em uma arquitetura SIMD com 4 elementos por vetor.

Para realizar a simulação da propagação do campo de onda ao longo do tempo, utilizamos dois arrays, um que armazena o instante atual da simulação e outro que armazena o instante anterior. Calculamos o próximo instante de tempo sobrescrevendo o instante anterior e o instante atual passa a ser o 
instante anterior com uma simples troca de ponteiros. Com isso mantemos o uso de memória em apenas dois arrays para armazenar o campo de onda.

Definimos o array onde o passo anterior e o próximo são armazenados como $U 1(n x, n y, n z)$ e o array que armazena o passo de tempo atual como $U 0(n x, n y, n z)$. Baseado na Figura 4.5a, no cálculo do ponto central, utilizamos os pontos em azul e o ponto central em vermelho do array U0 (instante atual) e apenas o ponto central do array $U 1$ (instante anterior).

Listagem 4.2: Pseudocódigo da propagação de onda.

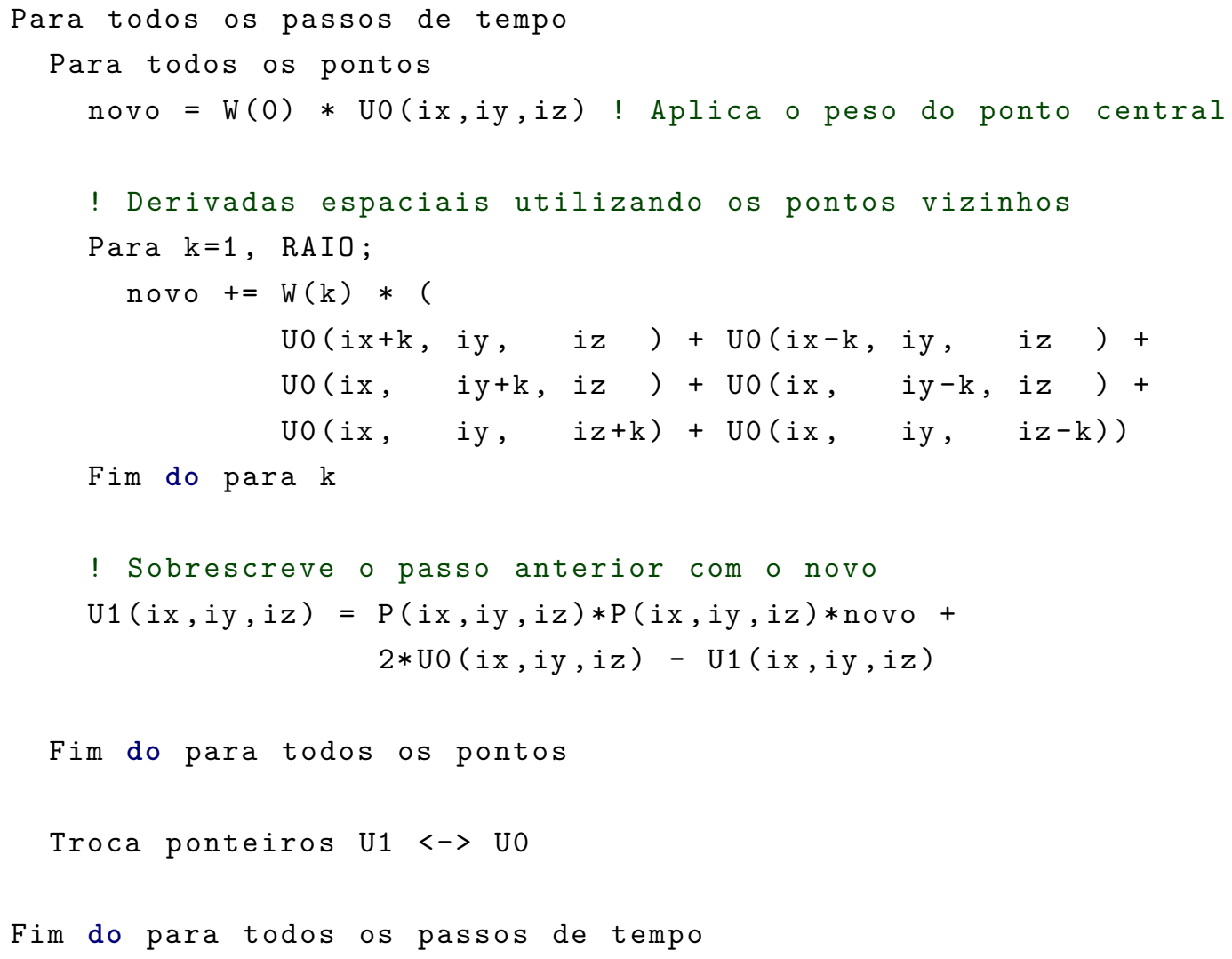

Na Listagem 4.2, $W(k)$ é o peso da soma ponderada para um ponto do estêncil com distância $k$ do ponto central. $P(i x, i y, i z)$ representa uma propriedade do modelo geológico. Neste caso a velocidade de propagação da onda. $U 0(i x, i y, i z)$ e $U 1(i x, i y, i z)$ são os arrays representando dois passos de tempo.

Podemos notar que, para o mesmo passo de tempo, os pontos podem ser calculados em paralelo de forma independente, mas não podemos iniciar o próximo passo em um ponto sem que todos os seus pontos vizinhos estejam calculados.

Utilizamos o OpenVec para para calcular simultaneamente vários pontos na direção $x$, conforme as Figuras 4.5b e 4.6. A dimensão $x$ está contígua em 
memória. Modificamos o tamanho $n x$ de todos os arrays para ser múltiplo de OV_FLOAT_WIDTH, garantindo com isso o alinhamento de memória.

Apenas os acessos com offset $i x \pm k$ podem estar desalinhados na memória, como por exemplo o termo U0(ix+k, iy, iz). Os acessos $i y \pm k$ saltam $\pm k \times n x$ elementos e os acessos $i z \pm k$ saltam $\pm k \times n x \times n y$ elementos. Como ambos os saltos são múltiplos de $n x$, que é múltiplo de OV_FLOAT_WIDTH, todos esses acessos são alinhados em memória.

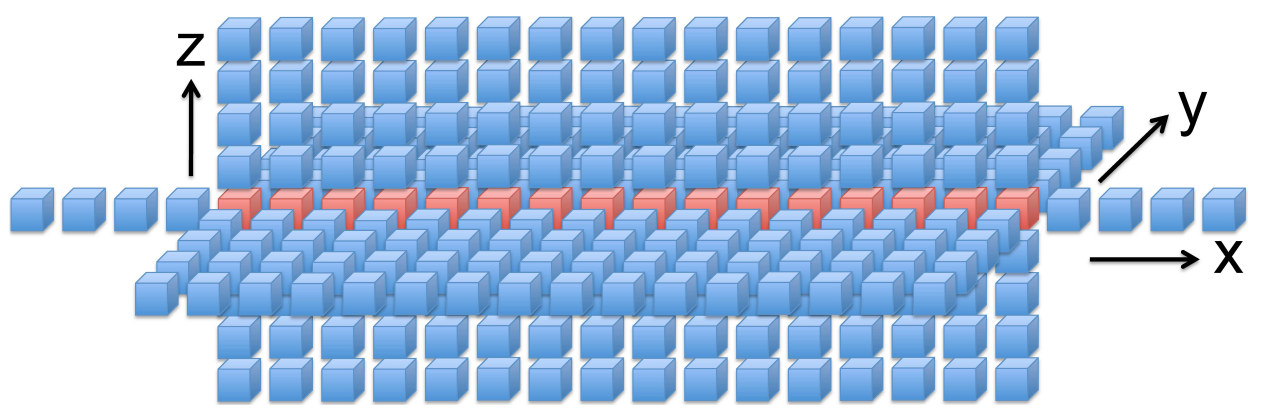

Figura 4.6: Cálculo simultâneo de 16 elementos em uma arquitetura SIMD com 16 elementos por vetor, como o Intel Xeon Phi.

Testamos o OpenVec na aplicação RTM (reverse time migration) (que também utiliza a HLIB) em um cluster com 200 nós dual socket AVX-2.

\section{6}

\section{Avaliação de Desempenho OpenVec}

Comparamos nossa implementação de um estêncil de $16^{\mathrm{a}}$ ordem com o trabalho referência[22]. Escolhemos este tamanho de estêncil para poder fazer uma comparação direta com o trabalho de referência. Implementamos o estêncil utilizando o OpenVec em ambas as linguagens $\mathrm{C}$ e $\mathrm{C}++$, com e sem unroll explícito. Tanto a nossa implementação, quanto a implementação de referência[22], utilizam o OpenMP para utilizar todos os núcleos da CPU. A Figura 4.7 mostra o comparativo de desempenho.

Nosso melhor resultado atingiu 2506 milhões de estêncis por segundo contra 2681 da implementação de referência. Atingimos $93 \%$ do desempenho da implementação de referência[22], que utiliza autotuning em conjunto com um algoritmo genético. O desempenho dos códigos em $\mathrm{C}$, que somente utilizam intrínsecos, foi melhor que o desempenho dos códigos em $\mathrm{C}++$, que utilizam os operadores matemáticos e intrínsecos.

O unroll explícito aumenta o desempenho no compilador gcc mas penaliza o desempenho no compilador da Intel.

Nossa implementação do estêncil faz parte dos exemplos de uso da distribuição do OpenVec. 


\begin{tabular}{|lr|}
\hline \multicolumn{2}{|c|}{ OpenVec } \\
Compilador & Msamples/s \\
gcc & 2217.1 \\
gcc unroll & 2413.3 \\
g++ unroll & 2147.7 \\
g++ & 2081.9 \\
icpc & 2389.8 \\
icpc unroll & 2321.0 \\
icc & 2506.1 \\
icc unroll & 2483.7 \\
\hline
\end{tabular}

\begin{tabular}{|c|c|}
\hline \multicolumn{2}{|c|}{ Código Referência AVX-2 } \\
\hline Compilador & Msamples/s \\
\hline icc & 2681.6 \\
\hline
\end{tabular}

Figura 4.7: Desempenho do estêncil de $16^{\mathrm{a}}$ ordem com OpenVec+OpenMP e com o código de referência, que utiliza intrínsecos AVX-2 e OpenMP. Ambiente do teste: processador Xeon E5-2697v3 @ 2.60 GHz com 14 núcleos, compilador da Intel versão 15.0.1.133 e gcc 4.4.7.

\section{7}

\section{Uso Combinado HLIB + OpenVec}

Neste capítulo exploramos o uso combinado da HLIB com o OpenVec. Utilizamos a aplicação migração reversa no tempo (RTM) para demonstrar o uso combinado dessas duas ferramentas. A Figura 4.8 mostra as camadas da RTM e a integração com a HLIB e o OpenVec.

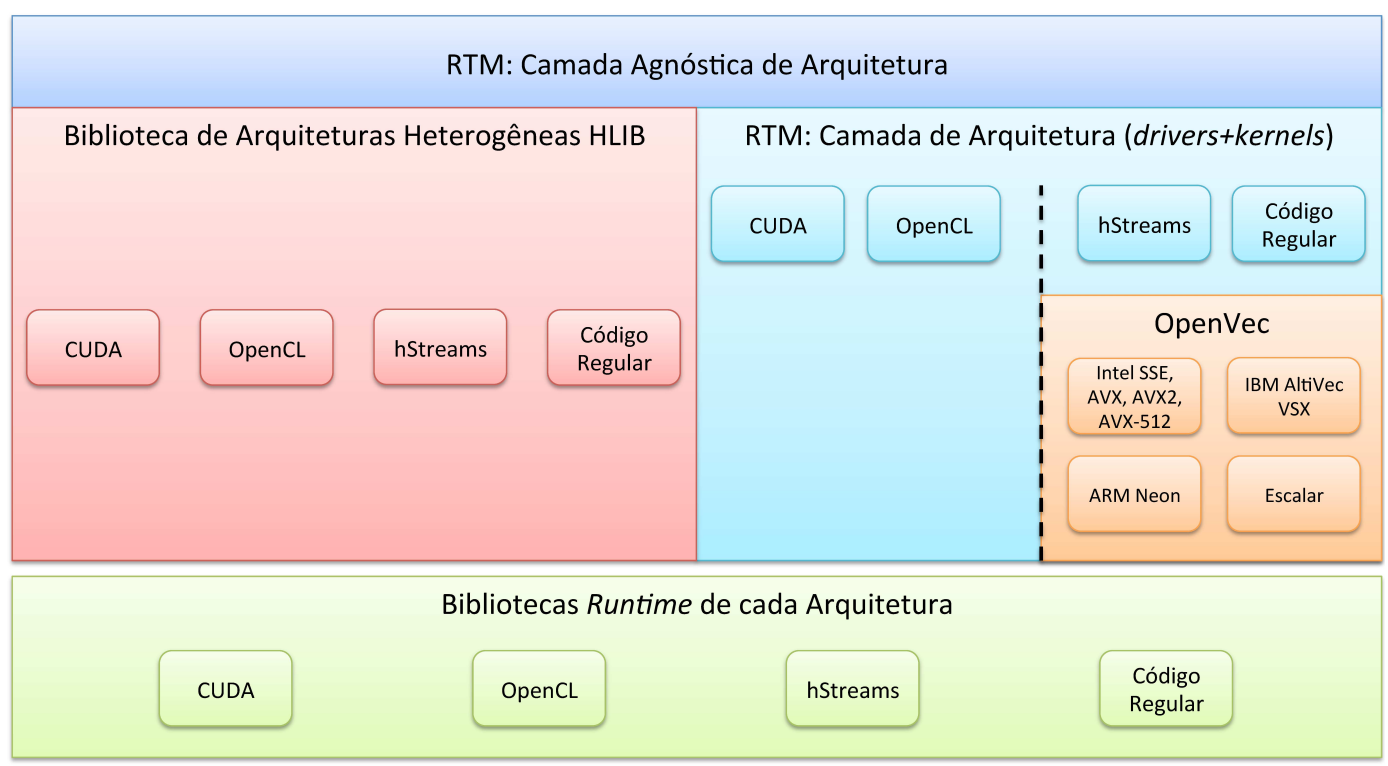

Figura 4.8: Camadas da RTM com HLIB+OpenVec. A RTM utiliza a HLIB para fazer o gerenciamento da computação heterogênea. Codificamos os kernels das arquiteturas hStreams e regular com o OpenVec. 
A Figura 4.9 mostra em detalhe a camada de arquitetura da RTM. Os drivers têm interfaces idênticas e compatíveis com Fortran 90. Cada driver repassa os argumentos e invoca seu respectivo kernel. O kernel OpenVec é agnóstico de arquitetura e pode executar em um dispositivo secundário como uma placa Xeon Phi ou em um processador tradicional, SIMD ou escalar.

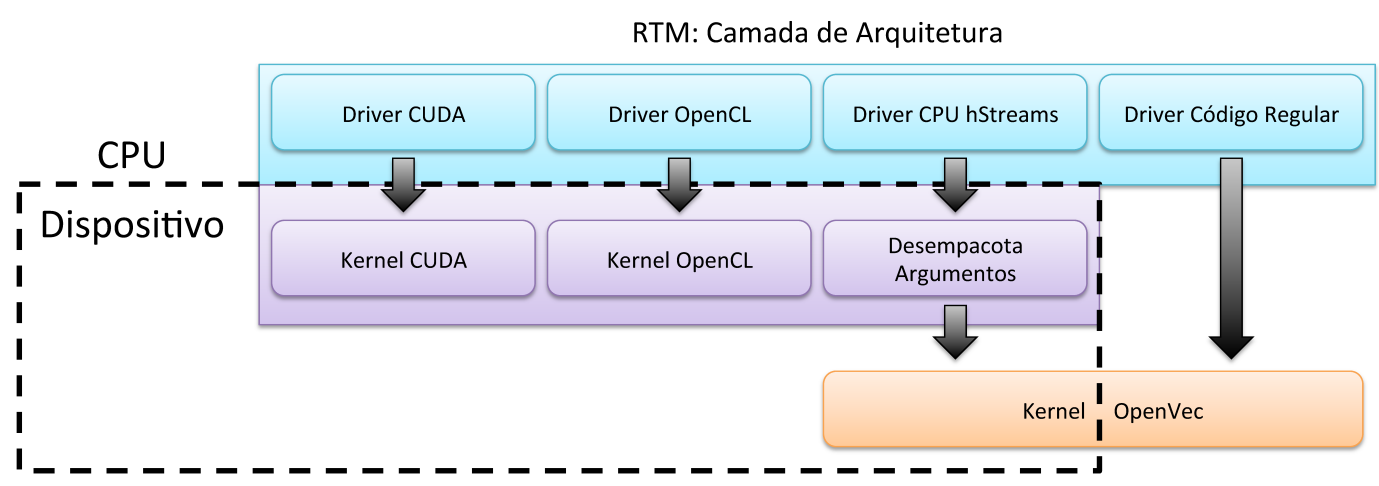

Figura 4.9: Camada de arquitetura da RTM. As setas indicam a direção de invocação. Por exemplo, o driver do código regular invoca o kernel OpenVec.

Suportamos as arquiteturas hStreams e regular com um único kernel, que utiliza o OpenVec. A invocação hStreams é diferente do código regular pois o kernel executa em um dispositivo enquanto que no código regular o kernel executa na CPU. Com isso, nosso driver hStreams, que executa na CPU, empacota os argumentos e dispara o kernel no dispositivo com a função hStreams_EnqueueCompute.

A maneira como o hStreams prepara os argumentos e dispara o kernel é similar ao OpenCL. Como mantivemos o mesmo kernel nos backends regular e hStreams. No backend hStreams foi necessário criar um código, que executa no dispositivo, e desempacota os argumentos antes de invocar o kernel, conforme a Figura 4.9. Este código de ligação mantem o kernel livre de chamadas proprietárias hStreams. A implementação atual do hStreams só permite argumentos do tipo uint64_t, por isso precisamos empacotar e desempacotar os argumentos nas invocações de kernels.

\section{8}

\section{Experimentos}

Comparamos o desempenho do estêncil de $16^{\mathrm{a}}$ ordem da Listagem $4.2 \mathrm{com}$ diversos dispositivos e APIs. A Tabela 4.2 mostra o desempenho em GFlops para cada API e dispositivo.

Executamos o nosso kernel CUDA no primeiro experimento. Este kernel possui otimizações específicas para arquiteturas de GPU iguais ou posteriores à 


\begin{tabular}{|l|l|l|c|c|}
\hline & API & Dispositivo & GFlops & Ano \\
\hline 1 & CUDA & Nvidia K80 (dual) & 751 & 2014 \\
\hline 2 & OpenCL & Nvidia K80 (dual) & 266 & 2014 \\
\hline 3 & OpenMP 4.0 & Nvidia K80 (dual) & 31 & 2014 \\
\hline 4 & OpenVec+OpenMP & Intel Xeon E5-2697v3@2.6 Ghz & 155 & 2014 \\
\hline 5 & OpenVec+OpenMP & Intel Xeon Phi 7120 & 262 & 2013 \\
\hline 6 & OpenCL & AMD Radeon HD 7970 & 236 & 2012 \\
\hline
\end{tabular}

Tabela 4.2: Experimentos com diversos dispositivos e APIs. A primeira coluna se refere ao número do experimento, e a última ao ano de lançamento do dispositivo.

Kepler. Como por exemplo, acessar a memória __shared__ utilizando palavras de 64bits[84] (cudaSharedMemBankSizeEightByte).

Os experimentos 2 e 6 tratam da execução de um kernel OpenCL inicialmente desenvolvido para suportar as GPUs da AMD. Executamos este kernel em uma GPU Nvidia (experimento 2) e em uma GPU AMD (experimento 6).

No experimento 3, utilizamos o OpenMP 4 sem a HLIB. Baixamos o trunk do projeto clang-omp. Geramos uma versão do compilador llvm com suporte ao OpenMP 4 com offload para GPUs Nvidia. O código OpenMP 4 ficou muito mais simples que o código HLIB+CUDA/OpenCL. Mas o desempenho ficou muito menor que as versões CUDA e OpenCL para o mesmo dispositivo (experimentos 1 e 2).

Traçamos um perfil da execução do experimento 3 com a ferramenta nvprof da Nvidia. Constatamos que a maior parte do tempo de execução foi gasto em cópias de memória entre a CPU e a GPU. Utilizamos a keyword target data para mover os dados para a GPU antes do loop de passos de tempo, e para mover os dados de volta apenas ao término do loop. Mas aparentemente, o compilador não respeitou a região target data, e transfere os dados em todas as iterações do loop.

No experimento 4, executamos o kernel OpenVec+OpenMP em um processador Xeon tradicional (homogêneo). Este processador possui instruções SIMD do tipo AVX-2. Este experimento é o mesmo da Figura 4.7, que atingiu 2506,1 milhões de amostras por segundo.

No experimento 5, executamos um kernel OpenVec+OpenMP otimizado para o Xeon Phi KNC. Utilizamos o Xeon Phi no modo offload com HLIB+hStreams. Esta versão do kernel utiliza o intrínseco ov_stream_stf, que equivale ao intrínseco _mm512_storenr_ps do Xeon Phi KNC. Como o hStreams ainda está na versão alfa (pré-beta) o desempenho poderá sofrer alterações em futuras versões.

Com exceção dos experimentos 2 e 3, em todos os outros experimentos 
otimizamos os kernels para o dispositivo do experimento.

\section{9}

\section{Discussão}

Com relação ao desempenho, as aplicações RTM e FWI não tiram proveito de nenhuma funcionalidade não implementada pela HLIB e pelo OpenVec. As decisões de projeto foram guiadas para atender estas duas aplicações. O uso das nossas ferramentas nestas aplicações trouxe apenas um ganho de portabilidade, pois iríamos implementá-las de forma nativa se não tivéssemos estas ferramentas.

Estas duas aplicações são muito utilizadas pela indústria de Óleo e Gás. Elas executam em parques computacionais com custos da ordem de dezenas de milhões de reais. Tentar extrair o máximo de desempenho de cada arquitetura traz um diferencial competitivo. Mas outras aplicações podem obter um desempenho maior utilizando funcionalidades presentes em apenas uma arquitetura. Para solucionar este problema, sugerimos o uso de extensões para contemplar estas aplicações. O uso das extensões adiciona chamadas específicas na camada agnóstica de arquitetura, mas uma parte do código ainda fica portável. 


\section{5}

\section{Conclusões}

A portabilidade e a manutenção de código são muito importantes no desenvolvimento de aplicações de alto desempenho, que podem ter uma vida útil de décadas[85, 86]. Por exemplo, há mais de 20 anos a Petrobras utiliza a Migração Kirchhoff, que foi otimizada ao longo dos anos para as seguintes arquiteturas: IBM Vector Facility, Silicon Graphics SMP, clusters de x86, IBM Cell[21] e clusters de GPUs Nvidia[42]

Como vimos, estas aplicações podem ser otimizadas para diferentes gerações de hardware. A nossa proposta de dividir a aplicação em uma camada de arquitetura e outra camada agnóstica de arquitetura ajudou a organizar e manter um código de alto desempenho para diversas arquiteturas.

Conseguimos, com a a nossa biblioteca HLIB, expor uma interface portável para as primitivas da computação heterogênea. Nosso foco foi manter a mesma abstração de APIs já existentes, como CUDA e OpenCL. Com isso, o desenvolvedor utiliza as mesmas primitivas que, por exemplo, em CUDA, mas escreve o código de gerenciamento uma única vez para todas as arquiteturas suportadas, incluindo a arquitetura tradicional que possui apenas a CPU.

Como a nossa biblioteca não é a responsável por escalonar os kernels e as movimentações entre a memória da CPU e a memória do dispositivo, fica a cargo do desenvolvedor atingir o desempenho potencial de cada arquitetura. Também cabe ao desenvolvedor implementar o kernel e o respectivo código driver (que enfileira o kernel) nas arquiteturas de interesse.

Demonstramos o uso da HLIB com GPUs Nvidia e a aplicação RTM de produção da Petrobras em um cluster de 2,6 petaflops de pico teórico. No kernel CUDA de propagação de onda, obtivemos 751 GFlops/s por placa Nvidia K80. Nas aplicações do Capítulo 4, não encontramos a necessidade de utilizar nenhuma funcionalidade não suportada pela HLIB. Portanto, não faz sentido avaliar o desempenho da HLIB, pois estaríamos medindo apenas o overhead da invocação Fortran, tendo em vista que os kernels estão implementados de forma nativa, e que a biblioteca é apenas um wrapper Fortran 90 para as APIs CUDA, OpenCL e hStreams. Mas pode haver uma perda de desempenho em uma aplicação que tira proveito de uma funcionalidade específica de uma determinada arquitetura.

A remoção de otimizações específicas para uma determinada arquitetura em uma aplicação de alto desempenho tende a deixar marcas no código, que vão tornando cada vez mais difícil a sua manutenção. Acreditamos que essa divisão em camadas e o uso da HLIB, além de facilitar a adição de novas arquiteturas, também facilita a remoção de arquiteturas. 
Como é difícil prever a evolução do mercado de aceleradores. Não há garantias de que a HLIB poderá ser portada para futuras gerações de arquiteturas heterogêneas.

O OpenVec atingiu o objetivo de implementar uma forma de vetorização explícita, que é agnóstica em relação a arquitetura e ao tamanho do vetor SIMD.

Se compararmos os códigos do estêncil OpenVec $\mathrm{C}++$ com a versão que utiliza intrínsecos proprietários, podemos notar um aumento na produtividade de codificação. Com o OpenVec $\mathrm{C}++$, o desenvolvedor escreve apenas um código para todas as arquiteturas, e o nosso overload de operadores facilita a codificação, a leitura e a manutenção do código. O desenvolvedor codifica de uma maneira próxima da tradicional (escalar), quando utiliza os operadores do OpenVec $\mathrm{C}++$.

Em termos de desempenho, o OpenVec apresentou baixo overhead pois utiliza macros para redefinir intrínsecos. Nossa implementação do estêncil de diferenças finitas com OpenMP+OpenVec atingiu 93\% (155 GFlops/s) do desempenho do estado da arte[22] para CPUs SIMD. Este desempenho, ainda fica distante dos 751 GFlops/s que obtivemos com uma GPU Nvidia K80 utilizando CUDA.

Testamos com sucesso nossa implementação em diversos sistemas operacionais, utilizando diversos compiladores, e em um conjunto de arquiteturas SIMD que compreendem os últimos 17 anos. Também testamos nossa implementação, através de um simulador, para a arquitetura Intel AVX-512 que ainda não foi lançada no mercado. Inspecionamos o código assembly para ter certeza que o compilador está gerando instruções vetoriais a partir de nossos intrínsecos e operadores.

Levamos apenas três dias para criar e adicionar diversos kernels OpenVec necessários para a aplicação RTM, que já utiliza a HLIB. Testamos com sucesso o uso combinado da HLIB+OpenVec na RTM, em um cluster com 200 nós, cada um com duas CPUs AVX 2.

Mas a vetorização explícita é apenas um dos tópicos necessários na difícil tarefa de otimização de código para as CPUs modernas. Cabe ao desenvolvedor explorar os demais tópicos como threading, blocking, array padding, loop unrolling, prefetching, aliasing, e NUMA+page awareness, e aplicar tudo isso com uma estratégia de autotuning. Podemos notar essa dificuldade analisando o desempenho do estêncil OpenVec para a mesma arquitetura (AVX 2), onde a versão com unroll explícito, teve um desempenho melhor em relação ao código sem unroll explícito nos compiladores gcc/g++, e um desempenho pior com os compiladores da Intel icc/icpc. 
Demonstramos que o uso da estratégia de dividir a aplicação em duas camadas em conjunto com o uso da HLIB (que implementa uma terceira camada) e do OpenVec auxiliaram na portabilidade de código em uma aplicação de alto desempenho. 


\section{Referências Bibliográficas}

[1] COURTLAND, R. Intel strikes back [news]. Spectrum, IEEE, v. 50, n. 8, p. 14-14, August 2013. ISSN 0018-9235.

[2] SHALF, J.; DOSANJH, S.; MORRISON, J. Exascale computing technology challenges. In: Proceedings of the 9th International Conference on High Performance Computing for Computational Science. Berlin, Heidelberg: Springer-Verlag, 2011. (VECPAR'10), p. 1-25. ISBN 978-3-642-19327-9. Disponível em: $<$ http://dl.acm.org/citation.cfm?id=1964238.1964240>.

[3] TOP500. TOP500 Supercomputer Site. 2016. Disponível em: $<$ http://www.top500.org $>$.

[4] HOSHINO, T. et al. CUDA vs OpenACC: Performance case studies with kernel benchmarks and a memory-bound cfd application. In: IEEE. Cluster, Cloud and Grid Computing (CCGrid), 2013 13th IEEE/ACM International Symposium on. [S.I.], 2013. p. 136-143.

[5] BAYSAL, E.; KOSLOFF, D. D.; SHERWOOD, J. W. Reverse time migration. Geophysics, Society of Exploration Geophysicists, v. 48, n. 11, p. 15141524, 1983.

[6] KRISHNAMURTHY, R. K. et al. High-performance and low-power challenges for sub-70 nm microprocessor circuits. In: Proc. IEEE Custom Integrated Circuits Conf. [S.I.: s.n.], 2002. v. 125, p. 12-15.

[7] HENNESSY, J. L.; PATTERSON, D. A. Computer Organization and Design (2Nd Ed.): The Hardware/Software Interface. San Francisco, CA, USA: Morgan Kaufmann Publishers Inc., 1998. 751 p. ISBN 1-55860428-6.

[8] FRANCHETTI, F. et al. Efficient utilization of SIMD extensions. Proceedings of the IEEE, v. 93, n. 2, p. 409-425, Feb 2005. ISSN 0018-9219.

[9] LARSEN, S.; AMARASINGHE, S. Exploiting superword level parallelism with multimedia instruction sets. In: Proceedings of the ACM SIGPLAN 2000 Conference on Programming Language Design and Implementation. New York, NY, USA: ACM, 2000. (PLDI '00), p. 145-156. ISBN 1-58113-199-2. Disponível em: $<$ http://doi.acm.org/10.1145/349299.349320>. 
[10] EICHENBERGER, A. E.; WU, P.; O'BRIEN, K. Vectorization for SIMD architectures with alignment constraints. In: ACM. ACM SIGPLAN Notices. [S.I.], 2004. v. 39, n. 6, p. 82-93.

[11] NUZMAN, D.; HENDERSON, R. Multi-platform auto-vectorization. In: IEEE COMPUTER SOCIETY. Proceedings of the International Symposium on Code Generation and Optimization. [S.I.], 2006. p. 281-294.

[12] WU, P.; EICHENBERGER, A. E.; WANG, A. Efficient SIMD code generation for runtime alignment and length conversion. In: IEEE. Code Generation and Optimization, 2005. CGO 2005. International Symposium on. [S.I.], 2005. p. 153-164.

[13] WU, P. et al. An integrated simdization framework using virtual vectors. In: ACM. Proceedings of the 19th annual international conference on Supercomputing. [S.I.], 2005. p. 169-178.

[14] NAISHLOS, D. et al. Vectorizing for a SIMdD DSP architecture. In: ACM. Proceedings of the 2003 international conference on Compilers, architecture and synthesis for embedded systems. [S.I.], 2003. p. 2-11.

[15] NUZMAN, D.; ROSEN, I.; ZAKS, A. Auto-vectorization of interleaved data for SIMD. In: ACM. ACM SIGPLAN Notices. [S.I.], 2006. v. 41, n. 6, p. 132-143.

[16] KARRENBERG, R.; HACK, S. Whole-function vectorization. In: IEEE. Code Generation and Optimization (CGO), 2011 9th Annual IEEE/ACM International Symposium on. [S.I.], 2011. p. 141-150.

[17] NUZMAN, D.; ZAKS, A. Outer-loop vectorization: revisited for short SIMD architectures. In: ACM. Proceedings of the 17th international conference on Parallel architectures and compilation techniques. [S.I.], 2008. p. 2-11.

[18] REN, G.; WU, P.; PADUA, D. An empirical study on the vectorization of multimedia applications for multimedia extensions. In: Parallel and Distributed Processing Symposium, 2005. Proceedings. 19th IEEE International. [S.I.: s.n.], 2005. p. 89b-89b.

[19] MALEKI, S. et al. An evaluation of vectorizing compilers. In: Parallel Architectures and Compilation Techniques (PACT), 2011 International Conference on. [S.I.: s.n.], 2011. p. 372-382. ISSN 1089-795X. 
[20] FRANCHETTI, F.; PUSCHEL, M. Short vector code generation for the discrete fourier transform. In: Parallel and Distributed Processing Symposium, 2003. Proceedings. International. [S.I.: s.n.], 2003. p. 10 pp.-. ISSN 1530-2075.

[21] PANETTA, J. et al. Computational characteristics of production seismic migration and its performance on novel processor architectures. In: Computer Architecture and High Performance Computing, 2007. SBAC-PAD 2007. 19th International Symposium on. [S.I.: s.n.], 2007. p. 11-18. ISSN 1550-6533.

[22] ANDREOLLI, C. et al. Chapter 23 - characterization and optimization methodology applied to stencil computations. In: JEFFERS, J. R. (Ed.). High Performance Parallelism Pearls. Boston: Morgan Kaufmann, 2015. p. 377 - 396. ISBN 978-0-12-802118-7. Disponível em: <http://www.sciencedirect.com/science/article/pii/B9780128021187000236>.

[23] ARAYA-POLO, M. et al. Assessing accelerator-based HPC reverse time migration. Parallel and Distributed Systems, IEEE Transactions on, v. 22, n. 1, p. 147-162, Jan 2011. ISSN 1045-9219.

[24] ZHENG, Y. et al. Accelerating with 512-bit SIMD : A case study for molecular dynamics simulation on Intel's Knights Corner. In: Communications and Information Technology (ICCIT), 2013 Third International Conference on. [S.I.: s.n.], 2013. p. 195-200.

[25] WILLIAMS, S. et al. Optimization of geometric multigrid for emerging multi-and manycore processors. In: IEEE COMPUTER SOCIETY PRESS. Proceedings of the International Conference on High Performance Computing, Networking, Storage and Analysis. [S.I.], 2012. p. 96.

[26] WILLIAMS, S. et al. Optimization of sparse matrix-vector multiplication on emerging multicore platforms. Parallel Computing, Elsevier, v. 35, n. 3, p. 178-194, 2009.

[27] TALLA, D. et al. Evaluating signal processing and multimedia applications on SIMD, VLIW and superscalar architectures. In: Computer Design, 2000. Proceedings. 2000 International Conference on. [S.I.: s.n.], 2000. p. 163-172. ISSN 1063-6404.

[28] KURZAK, J.; BUTTARI, A. Introduction to programming high performance applications on the Cell Broadband Engine. In: High-Performance Inter- 
connects, 2007. HOTI 2007. 15th Annual IEEE Symposium on. [S.I.: s.n.], 2007. p. 11-11. ISSN 1550-4794.

[29] WEST, N.; GEIGER, D.; SCHEETS, G. Accelerating software radio on ARM: Adding NEON support to VOLK. In: Radio and Wireless Symposium (RWS), 2015 IEEE. [S.I.: s.n.], 2015. p. 174-176.

[30] MITRA, G. et al. Use of SIMD vector operations to accelerate application code performance on low-powered ARM and intel platforms. In: Parallel and Distributed Processing Symposium Workshops PhD Forum (IPDPSW), 2013 IEEE 27th International. [S.I.: s.n.], 2013. p. 11071116.

[31] WILLIAMS, S. et al. The potential of the Cell processor for scientific computing. In: Proceedings of the 3rd Conference on Computing Frontiers. New York, NY, USA: ACM, 2006. (CF '06), p. 9-20. ISBN 1-59593-302-6. Disponível em: <http://doi.acm.org/10.1145/1128022.1128027>.

[32] WELCH, E. et al. A study of the use of SIMD instructions for two image processing algorithms. In: Image Processing Workshop (WNYIPW), 2012 Western New York. [S.I.: s.n.], 2012. p. 21-24.

[33] DATTA, K. et al. In: KURZAK, J.; BADER, D.; DONGARRA, J. (Ed.). Scientific Computing with Multicore and Accelerators. [S.I.]: Chapman and Hall/CRC, 2010. p. 234-235.

[34] SOUZA, P. et al. Chapter 24 - portable explicit vectorization intrinsics. In: JEFFERS, J. R. (Ed.). High Performance Parallelism Pearls. second. Boston: Morgan Kaufmann, 2015. p. 377 - 396. ISBN 978-0-12-802118-7. Disponível em: $<$ http://www.sciencedirect.com/science/article/pii/B9780128021187000236>.

[35] SOUZA, P. et al. OpenVec portable SIMD intrinsics. In: Second EAGE Workshop on High Performance Computing for Upstream. [S.I.: s.n.], 2015.

[36] SHAHBAHRAMI, A.; JUURLINK, B.; VASSILIADIS, S. Performance impact of misaligned accesses in SIMD extensions. In: Proceedings of the 17th Annual Workshop on Circuits, Systems and Signal Processing (ProRISC 2006). [S.I.: s.n.], 2006. p. 334-342.

[37] HARRIS, M. How to Access Global Memory Efficiently in CUDA $\mathbf{C} / \mathbf{C}+$ Kernels. 2013. Disponível em: 
$<$ http://devblogs.nvidia.com/parallelforall/how-access-global-memoryefficiently-cuda-c-kernels/ $>$.

[38] TILLET, P. et al. Towards performance-portable, scalable, and convenient linear algebra. In: HotPar. [S.I.: s.n.], 2013.

[39] BYUN, J.-H. et al. Autotuning sparse matrix-vector multiplication for multicore. ACM Trans. Math. Software, submitted, 2012.

[40] CABALLERO, D. et al. Optimizing fully anisotropic elastic propagation on intel xeon phi coprocessors. In: Second EAGE Workshop on High Performance Computing for Upstream. [S.I.: s.n.], 2015.

[41] MIT. The MIT License (MIT). Disponível em: $<$ https://opensource.org/licenses/MIT>.

[42] PANETTA, J. et al. Accelerating kirchhoff migration by CPU and GPU cooperation. In: Proceedings of the 2009 21st International Symposium on Computer Architecture and High Performance Computing. Washington, DC, USA: IEEE Computer Society, 2009. (SBAC-PAD '09), p. 26-32. ISBN 978-0-7695-3857-0. Disponível em: $<$ http://dx.doi.org/10.1109/SBAC-PAD.2009.29>.

[43] PANETTA, J. et al. Accelerating time and depth seismic migration by CPU and GPU cooperation. International Journal of Parallel Programming, Springer US, v. 40, n. 3, p. 290-312, 2012. ISSN 0885-7458. Disponível em: $<$ http://dx.doi.org/10.1007/s10766-011-0185-2>.

[44] MARTINS, L. et al. Accelerating curvature estimate in 3d seismic data using GPGPU. In: Computer Architecture and High Performance Computing (SBAC-PAD), 2014 IEEE 26th International Symposium on. [S.I.: s.n.], 2014. p. 105-111. ISSN 1550-6533.

[45] OWENS, J. et al. GPU computing. Proceedings of the IEEE, v. 96, n. 5, p. 879-899, May 2008. ISSN 0018-9219.

[46] NVIDIA. CUDA C Programming Guide. 2015. Disponível em: $<$ https://docs.nvidia.com/cuda/cuda-c-programming-guide/>.

[47] STEUWER, M.; KEGEL, P.; GORLATCH, S. Skelcl-a portable skeleton library for high-level GPU programming. In: IEEE. Parallel and Distributed Processing Workshops and Phd Forum (IPDPSW), 2011 IEEE International Symposium on. [S.I.], 2011. p. 1176-1182. 
[48] MARTINEZ, G.; GARDNER, M.; FENG, W.-c. Cu2cl: A CUDA-to-OpenCL translator for multi-and many-core architectures. In: IEEE. Parallel and Distributed Systems (ICPADS), 2011 IEEE 17th International Conference on. [S.I.], 2011. p. 300-307.

[49] DEMIDOV, D. et al. Programming CUDA and OpenCL: A case study using modern $\mathrm{C}++$ libraries. SIAM Journal on Scientific Computing, SIAM, v. 35, n. 5, p. C453-C472, 2013.

[50] REYES, R. et al. accull: An user-directed approach to heterogeneous programming. In: ISPA. IEEE, 2012. p. 654661. ISBN 978-1-4673-1631-6. Disponível em: <http://dblp.unitrier.de/db/conf/ispa/ispa2012.html\#ReyesLFS12>.

[51] HERNANDEZ, O. et al. Facing the multicore-challenge ii. In: KELLER, R.; KRAMER, D.; WEISS, J.-P. (Ed.). Berlin, Heidelberg: Springer-Verlag, 2012. cap. Experiences with High-level Programming Directives for Porting Applications to GPUs, p. 96-107. ISBN 978-3-642-30396-8. Disponível em: $<$ http://dl.acm.org/citation.cfm?id=2340646.2340659>.

[52] OpenMP Architecture Review Board. OpenMP Application Program Interface, Version 4.0. [S.I.], Julho 2013. Disponível em: $<$ http://openmp.org/wp/openmp-specifications/>.

[53] OpenACC Architecture Review Board. The OpenACC Application Programming Interface, Version 2.5. [S.I.], Outubro 2015. Disponível em: $<$ http://www.openacc.org/content/specifications-tech-reports>.

[54] MEDINA, D. S.; ST-CYR, A.; WARBURTON, T. OCCA: A unified approach to multi-threading languages. arXiv preprint arXiv:1403.0968, 2014.

[55] DOLBEAU, R.; BIHAN, S.; BODIN, F. HMPP: A hybrid multi-core parallel programming environment. In: Workshop on general purpose processing on graphics processing units (GPGPU 2007). [S.I.: s.n.], 2007. v. 28.

[56] DING, W. et al. Using GPU shared memory with a directive-based approach. In: Parallel Distributed Processing Symposium Workshops (IPDPSW), 2014 IEEE International. [S.I.: s.n.], 2014. p. 1021-1028.

[57] $\mathrm{GHOSH}$, S. et al. Experiences with OpenMP, PGI, HMPP and OpenACC directives on ISO/TTI kernels. In: High Performance Computing, Networking, Storage and Analysis (SCC), 2012 SC Companion:. [S.I.: s.n.], 2012. p. $691-700$. 
[58] FEKI, S. et al. Porting an explicit time-domain volume-integral-equation solver on GPUs with OpenACC [open problems in cem]. Antennas and Propagation Magazine, IEEE, v. 56, n. 2, p. 265-277, April 2014. ISSN 1045-9243.

[59] WIENKE, S. et al. OpenACC — first experiences with real-world applications. In: Euro-Par 2012 Parallel Processing. [S.I.]: Springer, 2012. p. 859-870.

[60] SOUZA, P.; BORGES, L.; NEWBURN, C. J. Heterogeneous architecture library. In: Second EAGE Workshop on High Performance Computing for Upstream. [S.I.: s.n.], 2015.

[61] NEWBURN, C. et al. Heterogeneous streaming. In: Sixth International Workshop on Accelerators and Hybrid Exascale. [S.I.: s.n.], 2016.

[62] AUGONNET, C. et al. StarPU: a unified platform for task scheduling on heterogeneous multicore architectures. Concurrency and Computation: Practice and Experience, Wiley Online Library, v. 23, n. 2, p. 187-198, 2011.

[63] LUK, C.-K.; HONG, S.; KIM, H. Qilin: exploiting parallelism on heterogeneous multiprocessors with adaptive mapping. In: IEEE. Microarchitecture, 2009. MICRO-42. 42nd Annual IEEE/ACM International Symposium on. [S.I.], 2009. p. 45-55.

[64] BOSILCA, G. et al. DAGuE: A generic distributed DAG engine for high performance computing. Parallel Computing, Elsevier, v. 38, n. 1, p. 3751, 2012.

[65] CAO, C. et al. cIMAGMA: High performance dense linear algebra with OpenCL. In: ACM. Proceedings of the International Workshop on OpenCL 2013 \& 2014. [S.I.], 2014. p. 1.

[66] WANG, L. et al. Scaling scientific applications on clusters of hybrid multicore/GPU nodes. In: ACM. Proceedings of the 8th ACM international conference on computing frontiers. [S.I.], 2011. p. 6.

[67] CHEN, Y.; CUI, X.; MEI, H. Large-scale FFT on GPU clusters. In: ACM. Proceedings of the 24th ACM International Conference on Supercomputing. [S.I.], 2010. p. 315-324.

[68] PEÑA, A. J. et al. A complete and efficient CUDA-sharing solution for HPC clusters. Parallel Computing, Elsevier, v. 40, n. 10, p. 574-588, 2014. 
[69] CALANDRA, $H$. et al. Evaluation of successive CPUs/APUs/GPUs based on an OpenCL finite difference stencil. In: Parallel, Distributed and Network-Based Processing (PDP), 2013 21st Euromicro International Conference on. [S.I.: s.n.], 2013. p. 405-409. ISSN 1066-6192.

[70] Message Passing Interface Forum. MPI: A Message-Passing Interface Standard. Knoxville, TN, USA, 1998. Disponível em: <http://www.mpiforum.org/docs/mpi-1.1/mpi-11-html/mpi-report.html $>$.

[71] BERNASCHI, M.; BISSON, M.; ROSSETTI, D. Benchmarking of communication techniques for GPUs. Journal of Parallel and Distributed Computing, v. 73, n. 2, p. 250 - 255, 2013. ISSN 0743-7315. Disponível em: <http://www.sciencedirect.com/science/article/pii/S0743731512002213>.

[72] MICIKEVICIUS, P. Multi-gpu programming. GPU Computing Webinars, NVIDIA, 2011.

[73] CUDA-Aware OpenMPI distribution. [S.I.]. Disponível em: $<$ https://www.open-mpi.org/faq/?category=buildcuda $>$.

[74] CUDA-Aware MVAPICH2 distribution. [S.I.]. Disponível em: $<$ http://mvapich.cse.ohio-state.edu/features/\#mv2gdr>.

[75] ZHU, J.; LINES, L. R. Comparison of kirchhoff and reverse-time migration methods with applications to prestack depth imaging of complex structures. GEOPHYSICS, v. 63, n. 4, p. 1166-1176, 1998. Disponível em: $<$ http://dx.doi.org/10.1190/1.1444416>.

[76] ZENG, C. et al. Broadband least-squares reverse time migration for complex structure imaging. SEG 84th Annual International Meeting, Society of Exploration Geophysicists, p. 3715-3719, 2014.

[77] VERMEER, G. J.; BEASLEY, C. J. 3-D seismic survey design. [S.I.]: Society of Exploration Geophysicists Tulsa, 2002.

[78] TARANTOLA, A. Inversion of seismic reflection data in the acoustic approximation. Geophysics, Society of Exploration Geophysicists, v. 49, n. 8, p. 1259-1266, 1984.

[79] GAUTHIER, O.; VIRIEUX, J.; TARANTOLA, A. Two-dimensional nonlinear inversion of seismic waveforms: Numerical results. Geophysics, Society of Exploration Geophysicists, v. 51, n. 7, p. 1387-1403, 1986. 
[80] MORA, P. Nonlinear two-dimensional elastic inversion of multioffset seismic data. Geophysics, Society of Exploration Geophysicists, v. 52, n. 9, p. 12111228, 1987.

[81] CRASE, E. et al. Robust elastic nonlinear waveform inversion: Application to real data. Geophysics, Society of Exploration Geophysicists, v. 55, n. 5, p. $527-538,1990$.

[82] KAPOOR, S. et al. Full waveform inversion around the world. In: 75th EAGE Conference \& Exhibition incorporating SPE EUROPEC 2013. [S.I.: s.n.], 2013.

[83] VIRIEUX, J.; OPERTO, S. An overview of full-waveform inversion in exploration geophysics. GEOPHYSICS, Society of Exploration Geophysicists, v. 74, n. 6, p. WCC1-WCC26, nov. 2009. Disponível em: $<$ http://dx.doi.org/10.1190/1.3238367>.

[84] Nvidia. Kepler Tuning Guide. 2015. Disponível em: $<$ http://docs.nvidia.com/cuda/kepler-tuning-guide/\#shared-memorybandwidth $>$.

[85] KENDALL, R. P. et al. A proposed taxonomy for software development risks for high-performance computing (HPC) scientific/engineering applications. [S.I.], 2007.

[86] VANTER, M. L. V. D.; POST, D.; ZOSEL, M. E. HPC needs a tool strategy. In: ACM. Proceedings of the second international workshop on Software engineering for high performance computing system applications. [S.I.], 2005. p. 55-59. 


\section{A}

\section{Documentação OpenVec}

Para utilizar o OpenVec o desenvolvedor deve adicionar o header file do OpenVec conforme o código a seguir:

\#include"openvec.h"

\section{A.1}

\section{Como Compilar Código OpenVec}

No momento da compilação o header file openvec $\cdot \mathrm{h}$ detecta a arquitetura SIMD baseada nas flags do compilador. Para desligar a detecção automática basta adicionar a flag -D_OV_NOAUTO. A Tabela A.1 mostra como desligar a detecção automática e forçar a compilação para uma determinada arquitetura.

\begin{tabular}{|l|l|l|l|}
\hline Flag OpenVec & Arquitetura & Compilador & Flag Arquitetura \\
\hline -D_OV_NOAUTO -D_OV_MIC & Intel KNC KNF & Intel C/C++ & -mmic \\
\hline -D_OV_NOAUTO -D_OV_AVX & Intel AVX & Intel C/C++ & -xAVX \\
\hline -D_OV_NOAUTO -D_OV_AVX2 & Intel AVX 2 & Intel C/C++ & -xCORE-AVX2 \\
\hline -D_OV_NOAUTO -D_OV_AVX512 & Intel AVX 512 & Intel C/C++ & -xCORE-AVX512 \\
\hline -D_OV_NOAUTO -D_OV_AVX & Intel AVX & gcc/llvm & -mavX \\
\hline -D_OV_NOAUTO -D_OV_AVX2 & Intel AVX 2 & gcc/llvm & -mavx2 \\
\hline -D_OV_NOAUTO -D_OV_SSE & Intel SSE & gcc/llvm & -msse \\
\hline -D_OV_NOAUTO -D_OV_SSE4 & Intel SSE4 & gcc/llvm & -msse4 \\
\hline -D_OV_NOAUTO -D_OV_SSE & Intel SSE & Intel C/C++ & -xSSE \\
\hline -D_OV_NOAUTO -D_OV_SSE4 & Intel SSE4 & Intel C/C++ & -xSSE4.1 \\
\hline -D_OV_NOAUTO -D_OV_NEON & ARM Neon & gcc/llvm & -mfpu=neon \\
\hline -D_OV_NOAUTO -D_OV_NEON & ARM Neon 64 bit & gcc/llvm & \\
\hline -D_OV_NOAUTO -D_OV_ALTIVEC & IBM Altivec & gcc & -maltivec \\
\hline -D_OV_NOAUTO & Escalar & qualquer & qualquer \\
\hline
\end{tabular}

Tabela A.1: Flags de compilação para diferentes compiladores e arquiteturas.

Em todos os nossos testes a detecção automática funcionou, mas disponibilizamos uma maneira de forçar a compilação para uma determinada arquitetura.

O código do OpenVec escrito em $\mathrm{C}$ respeita o padrão C89, mas alguns exemplos fornecidos com o o OpenVec utilizam características do padrão C99. Para compilar estes códigos de exemplo é necessário adicionar a flag-std=c99.

\section{A. 2 \\ Operadores}

Em $\mathrm{C}++$ o OpenVec faz um overload dos principais operadores. As operações matemáticas básicas com variáveis SIMD estão definidas, conforme o exemplo a seguir: 


$$
\begin{aligned}
& \text { ov_float a,b,c,d;//SIMD } \\
& \text { float e;// escalar } \\
& d=a * c-(a+b) / e+2.0 f * c ;
\end{aligned}
$$

No exemplo podemos notar que as operações entre vetores SIMD "a*c" e " $a+b "$ estão definidas, bem como as operações entre vetores e escalares " $(\mathrm{a}+\mathrm{b}) / \mathrm{e} " \mathrm{e} " 2.0 \mathrm{f} * \mathrm{c} "$.

Em toda operação entre um vetor SIMD e um escalar, o escalar é promovido a vetor, criando um vetor temporário onde todos os elementos são iguais ao escalar, conforme o exemplo a seguir.

$$
2.0 \mathrm{f} * \mathrm{c} ; \quad / / \quad 2 * c_{0} ; 2 * c_{1} ; 2 * c_{2} ; \ldots ; 2 * c_{n} ;
$$

\section{A.3 \\ Constantes}

Definimos em tempo de compilação todas as constantes OpenVec para uma única arquitetura SIMD. As flags de compilação definem a arquitetura.

\section{OV_COMPILER}

Contem uma string com o nome do compilador, por exemplo "gcc".

\section{OV_DOUBLE_WIDTH}

Contem a largura SIMD para o tipo ov_double, ou seja, variáveis do tipo ov_double possuem OV_DOUBLE_WIDTH doubles.

\section{OV_DOUBLE_TAIL}

Contem a largura SIMD menos um elemento, esta constante facilita o tratamento da cauda de vetores.

\section{OV_FLOAT_WIDTH}

Contem a largura SIMD para o tipo ov_float, ou seja, variáveis do tipo ov_float possuem OV_FLOAT_WIDTH floats.

\section{OV_FLOAT_TAIL}

Contem a largura SIMD menos um elemento, esta constante facilita o tratamento da cauda de vetores. 


\section{OV_PLATFORM}

Contem uma string com o nome da plataforma SIMD, por exemplo "Intel COMPILER AVX 2"

\section{ov_restrict}

O OpenVec define a macro ov_restrict que funciona como o restrict do padrão C99. Esta macro pode ser utilizada tanto em $\mathrm{C}$ como em $\mathrm{C}++$, definimos nossa macro para cada linguagem e compilador. Quando o compilador não suporta a definição restrict a macro não tem nenhum efeito.

O exemplo a seguir mostra o uso da macro ov_restrict:

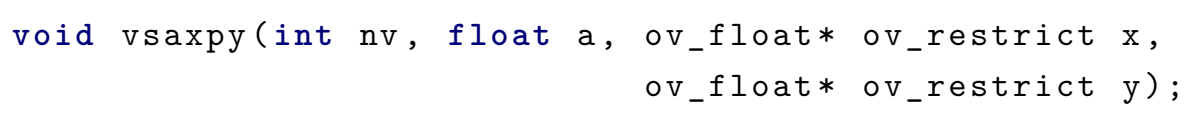

O desenvolvedor deve sempre que possível utilizar esta macro, pois ela habilita o compilador a assumir certas otimizações que podem gerar um código com maior desempenho.

\section{A.4 \\ Funções}

As funções OpenVec atuam em variáveis SIMD do tipo ov_float e ov_double, a documentação das funções vetoriais mostra em detalhe a operação executada nos elementos da variável vetorial.

O exemplo a seguir mostra o cálculo simultâneo SIMD da raiz quadrada em todos os elementos da variável vetorial "a" utilizando a notação $a_{0 . . n}$ para representar seus elementos.

$\sqrt{a_{0}} ; \sqrt{a_{1}} ; \sqrt{a_{2}} ; \ldots ; \sqrt{a_{n}}$

Essa notação é utilizada durante toda a documentação das funções. Por exemplo, uma variável "ov_float a", em uma arquitetura ARM Neon, possui os seguintes elementos: $\left\{a_{0}, a_{1}, a_{2}, a_{3}\right\}$, onde o limite $n=3$ se refere a constante OV_FLOAT_WIDTH-1. Analogamente em uma variável "ov_double a", $n$ refere-se a constante OV_DOUBLE_WIDTH-1.

Algumas funções possuem várias interfaces fazendo um overload de argumentos, essas funções estão disponíveis apenas em $\mathrm{C}++$ e são marcadas na documentação conforme o exemplo a seguir para a função ceil:

$$
\operatorname{ceil}^{C++}
$$


ov_calloc

void *ov_calloc(size_t nmemb, size_t size)

Aloca uma área contígua de memória de tamanho nmemb $\times$ size bytes, zera todos os bytes e retorna o endereço alocado.

ov_malloc

void *ov_malloc(size_t size)

Aloca uma área contígua de memória de tamanho size bytes e retorna o endereço alocado.

$\operatorname{ceil}^{C++}$

ov_double ceil(ov_double const a)

Retorna um vetor SIMD de doubles arredondando para o maior inteiro mais próximo de forma independente para todos os elementos de a.

$\left\lceil a_{0}\right\rceil ;\left\lceil a_{1}\right\rceil ;\left\lceil a_{2}\right\rceil ; \ldots ;\left\lceil a_{n}\right\rceil ;$

$\operatorname{ceilf}^{C++}$

ov_float ceilf(ov_float const a)

Retorna um vetor SIMD de floats arredondando para o maior inteiro mais próximo de forma independente para todos os elementos de a.

$\left\lceil a_{0}\right\rceil ;\left\lceil a_{1}\right\rceil ;\left\lceil a_{2}\right\rceil ; \ldots ;\left\lceil a_{n}\right\rceil ;$

$\mathrm{fabs}^{\mathrm{C}++}$

ov_double fabs(ov_double const a)

Retorna um vetor SIMD de doubles com a operação módulo aplicada de forma independente em todos os elementos de a.

$\left|a_{0}\right| ;\left|a_{1}\right| ;\left|a_{2}\right| ; \ldots ;\left|a_{n}\right|$ 
fabsf ${ }^{C++}$

ov_float fabsf(ov_float const a)

Retorna um vetor SIMD de floats com a operação módulo aplicada de forma independente em todos os elementos de a.

$\left|a_{0}\right| ;\left|a_{1}\right| ;\left|a_{2}\right| ; \ldots ;\left|a_{n}\right|$

floor ${ }^{C++}$

ov_double floor(ov_double const a)

Retorna um vetor SIMD de doubles arredondando para o menor inteiro mais próximo de forma independente para todos os elementos de a.

$\left\lfloor a_{0}\right\rfloor ;\left\lfloor a_{1}\right\rfloor ;\left\lfloor a_{2}\right\rfloor ; \ldots ;\left\lfloor a_{n}\right\rfloor$

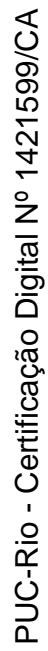

floorf ${ }^{C++}$

ov_float floorf(ov_float const a)

Retorna um vetor SIMD de floats arredondando para o menor inteiro mais próximo de forma independente para todos os elementos de a.

$\left\lfloor a_{0}\right\rfloor ;\left\lfloor a_{1}\right\rfloor ;\left\lfloor a_{2}\right\rfloor ; \ldots ;\left\lfloor a_{n}\right\rfloor$

ov_absd

ov_double ov_absd(ov_double const a)

Retorna um vetor SIMD de doubles com a operação módulo aplicada de forma independente em todos os elementos de a.

$\left|a_{0}\right| ;\left|a_{1}\right| ;\left|a_{2}\right| ; \ldots ;\left|a_{n}\right|$

ov_absf

ov_float ov_absf(ov_float const a)

Retorna um vetor SIMD de floats com a operação módulo aplicada de forma independente em todos os elementos de a. 
$\left|a_{0}\right| ;\left|a_{1}\right| ;\left|a_{2}\right| ; \ldots ;\left|a_{n}\right|$

ov_addd

ov_double ov_addd(ov_double const a, ov_double const b)

Retorna um vetor SIMD de doubles com a operação adição aplicada de forma independente elemento a elemento de a e b.

$a_{0}+b_{0} ; a_{1}+b_{1} ; a_{2}+b_{2} ; \ldots ; a_{n}+b_{n} ;$

ov_addf

ov_float ov_addf(ov_float const a, ov_float const b)

Retorna um vetor SIMD de floats com a operação adição aplicada de forma independente elemento a elemento de a e b.

$a_{0}+b_{0} ; a_{1}+b_{1} ; a_{2}+b_{2} ; \ldots ; a_{n}+b_{n} ;$

ov_all_ge_Od

ov_bool ov_all_ge_Od(ov_double a)

Retorna verdadeiro se todos os elementos de a são maiores ou iguais a zero.

$\operatorname{all}\left(a_{0} \geq 0, a_{1} \geq 0, a_{2} \geq 0, \ldots, a_{n} \geq 0\right)$

ov_all_ge_of

ov_bool ov_all_ge_Of(ov_float a)

Retorna verdadeiro se todos os elementos de a são maiores ou iguais a zero.

$\operatorname{all}\left(a_{0} \geq 0, a_{1} \geq 0, a_{2} \geq 0, \ldots, a_{n} \geq 0\right)$ 
ov_all_lt_od

ov_bool ov_all_lt_Od(ov_double a)

Retorna verdadeiro se todos os elementos de a são menores que zero.

$\operatorname{all}\left(a_{0}<0, a_{1}<0, a_{2}<0, \ldots, a_{n}<0\right)$

ov_all_lt_of

ov_bool ov_all_lt_of (ov_float a)

Retorna verdadeiro se todos os elementos de a são menores que zero.

$\operatorname{all}\left(a_{0}<0, a_{1}<0, a_{2}<0, \ldots, a_{n}<0\right)$

ov_all_maxd

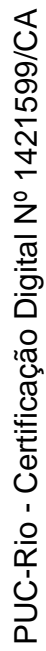

double ov_all_maxd(ov_double const a)

Retorna um escalar double com o maior elemento de a.

$\max \left(a_{0}, a_{1}, a_{2}, \ldots, a_{n}\right)$

ov_all_maxf

float ov_all_maxf(ov_float const a)

Retorna um escalar float com o maior elemento de a.

$\max \left(a_{0}, a_{1}, a_{2}, \ldots, a_{n}\right)$

ov_all_mind

double ov_all_mind(ov_double const a)

Retorna um escalar double com o menor elemento de a.

$\min \left(a_{0}, a_{1}, a_{2}, \ldots, a_{n}\right)$ 
ov_all_minf

float ov_all_minf(ov_float const a)

Retorna um escalar float com o menor elemento de a.

$\min \left(a_{0}, a_{1}, a_{2}, \ldots, a_{n}\right)$

ov_all_prodd

double ov_all_prodd(ov_double const a)

Retorna um escalar double com o produto de todos os elementos de a.

$a_{0} \times a_{1} \times a_{2} \times \ldots \times a_{n}$

ov_all_prodf

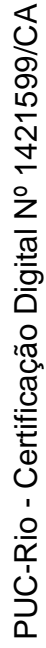

float ov_all_prodf(ov_float const a)

Retorna um escalar float com o produto de todos os elementos de a.

$a_{0} \times a_{1} \times a_{2} \times \ldots \times a_{n}$

ov_all_sumd

double ov_all_sumd (ov_double const a)

Retorna um escalar double com a soma de todos os elementos de a.

$a_{0}+a_{1}+a_{2}+\ldots+a_{n}$

ov_all_sumf

float ov_all_sumf (ov_float const a)

Retorna um escalar float com a soma de todos os elementos de a.

$a_{0}+a_{1}+a_{2}+\ldots+a_{n}$ 
ov_alld

ov_bool ov_alld(ov_maskd const mask)

Retorna verdadeiro se todos os bits de mask estiverem ligados.

ov_allf

ov_bool ov_allf(ov_maskf const mask)

Retorna verdadeiro se todos os bits de mask estiverem ligados.

ov_any_ge_Od

ov_bool ov_any_ge_Od(a)

Retorna verdadeiro se ao menos um elemento de a é maior ou igual a zero.

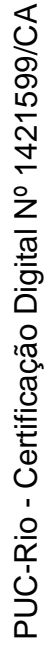

$\operatorname{any}\left(a_{0} \geq 0 ; a_{1} \geq 0 ; a_{2} \geq 0 ; \ldots ; a_{n} \geq 0\right)$

ov_any_ge_of

ov_bool ov_any_ge_of (a)

Retorna verdadeiro se ao menos um elemento de a é maior ou igual a zero.

$\operatorname{any}\left(a_{0} \geq 0 ; a_{1} \geq 0 ; a_{2} \geq 0 ; \ldots ; a_{n} \geq 0\right)$

ov_any_lt_od

ov_bool ov_any_lt_Od(a)

Retorna verdadeiro se ao menos um elemento de a é menor que zero.

$\operatorname{any}\left(a_{0}<0 ; a_{1}<0 ; a_{2}<0 ; \ldots ; a_{n}<0\right)$

ov_any_lt_of

ov_bool ov_any_lt_Of(a)

Retorna verdadeiro se ao menos um elemento de a é menor que zero.

$\operatorname{any}\left(a_{0}<0 ; a_{1}<0 ; a_{2}<0 ; \ldots ; a_{n}<0\right)$ 
ov_anyd

ov_bool ov_anyd (ov_maskd const mask)

Retorna verdadeiro se pelo menos um bit de mask estiver ligado.

ov_anyf

ov_bool ov_anyf (ov_maskf const mask)

Retorna verdadeiro se pelo menos um bit de mask estiver ligado.

ov_ceild

ov_double ov_ceild(ov_double const a)

Retorna um vetor SIMD de doubles arredondando para o maior inteiro mais próximo de forma independente para todos os elementos de a.

$\left\lceil a_{0}\right\rceil ;\left\lceil a_{1}\right\rceil ;\left\lceil a_{2}\right\rceil ; \ldots ;\left\lceil a_{n}\right\rceil ;$

ov_ceilf

ov_float ov_ceilf(ov_float const a)

Retorna um vetor SIMD de floats arredondando para o maior inteiro mais próximo de forma independente para todos os elementos de a.

$\left\lceil a_{0}\right\rceil ;\left\lceil a_{1}\right\rceil ;\left\lceil a_{2}\right\rceil ; \ldots ;\left\lceil a_{n}\right\rceil ;$

ov_conditionald

ov_double ov_conditionald(ov_maskd const mask, ov_double const a, ov_double const b)

Retorna um vetor SIMD de doubles mantendo os elementos de a onde a máscara é verdadeira e os elementos de b onde a máscara é falsa. 
ov_conditionalf

ov_float ov_conditionalf(ov_maskf const mask, ov_float const a, ov_float const b)

Retorna um vetor SIMD de floats mantendo os elementos de a onde a máscara é verdadeira e os elementos de b onde a máscara é falsa.

ov_divd

ov_double ov_divd(ov_double const a, ov_double const b)

Retorna um vetor SIMD de doubles com a operação divisão aplicada de forma independente elemento a elemento de a e b.

$\frac{a_{0}}{b_{0}} ; \frac{a_{1}}{b_{1}} ; \frac{a_{2}}{b_{2}} ; \ldots ; \frac{a_{n}}{b_{n}} ;$

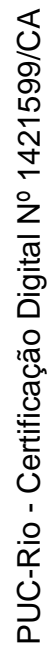

ov_divf

ov_float ov_divf(ov_float const a, ov_float const b)

Retorna um vetor SIMD de floats com a operação divisão aplicada de forma independente elemento a elemento de a e b.

$\frac{a_{0}}{b_{0}} ; \frac{a_{1}}{b_{1}} ; \frac{a_{2}}{b_{2}} ; \ldots ; \frac{a_{n}}{b_{n}}$

ov_eqd

ov_maskd ov_eqd(ov_double const a, ov_double const b)

Retorna uma máscara de bits com a operação == aplicada de forma independente elemento a elemento de a e b.

$a_{0}==b_{0} ; a_{1}==b_{1} ; a_{2}==b_{2} ; \ldots ; a_{n}==b_{n} ;$

ov_eqf

ov_maskf ov_eqf(ov_float const a, ov_float const b)

Retorna uma máscara de bits com a operação == aplicada de forma independente elemento a elemento de a e b. 
$a_{0}==b_{0} ; a_{1}==b_{1} ; a_{2}==b_{2} ; \ldots ; a_{n}==b_{n} ;$

ov_floord

ov_double ov_floord(ov_double const a)

Retorna um vetor SIMD de doubles arredondando para o menor inteiro mais próximo de forma independente para todos os elementos de a.

$\left\lfloor a_{0}\right\rfloor ;\left\lfloor a_{1}\right\rfloor ;\left\lfloor a_{2}\right\rfloor ; \ldots ;\left\lfloor a_{n}\right\rfloor$

ov_floorf

ov_float ov_floorf(ov_float const a)

Retorna um vetor SIMD de floats arredondando para o menor inteiro mais próximo de forma independente para todos os elementos de a.

$\left\lfloor a_{0}\right\rfloor ;\left\lfloor a_{1}\right\rfloor ;\left\lfloor a_{2}\right\rfloor ; \ldots ;\left\lfloor a_{n}\right\rfloor$

ov_free

void ov_free(void *ptr)

Desaloca uma memória alocada previamente com a função ov_malloc ou ov_calloc.

ov_ged

ov_maskd ov_ged(ov_double const a, ov_double const b)

Retorna uma máscara de bits com a operação $\geq$ aplicada de forma independente elemento a elemento de a e b.

$a_{0} \geq b_{0} ; a_{1} \geq b_{1} ; a_{2} \geq b_{2} ; \ldots ; a_{n} \geq b_{n} ;$

ov_gef

ov_maskf ov_gef(ov_float const a, ov_float const b) 
Retorna uma máscara de bits com a operação $\geq$ aplicada de forma independente elemento a elemento de a e b.

$a_{0} \geq b_{0} ; a_{1} \geq b_{1} ; a_{2} \geq b_{2} ; \ldots ; a_{n} \geq b_{n} ;$

ov_getzerod

ov_double ov_getzerod()

Retorna um vetor SIMD de doubles com todos os valores zerados.

ov_getzerof

ov_float ov_getzerof()

Retorna um vetor SIMD de floats com todos os valores zerados.

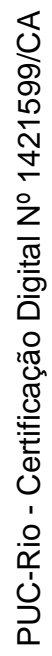

ov_gtd

ov_maskd ov_gtd(ov_double const a, ov_double const b)

Retorna uma máscara de bits com a operação > aplicada de forma independente elemento a elemento de a e b.

$a_{0}>b_{0} ; a_{1}>b_{1} ; a_{2}>b_{2} ; \ldots ; a_{n}>b_{n}$

ov_gtf

ov_maskf ov_gtf (ov_float const a, ov_float const b)

Retorna uma máscara de bits com a operação > aplicada de forma independente elemento a elemento de a e b.

$a_{0}>b_{0} ; a_{1}>b_{1} ; a_{2}>b_{2} ; \ldots ; a_{n}>b_{n}$

ov_ldd

ov_double ov_ldd(double const $*$ addr)

Retorna um vetor SIMD de doubles carregado a partir de um endereço alinhado em memória. 
ov_ldf

ov_float ov_ldf(float const $*$ addr $)$

Retorna um vetor SIMD de floats carregado a partir de um endereço alinhado em memória.

ov_led

ov_maskd ov_led(ov_double const a, ov_double const b)

Retorna uma máscara de bits com a operação $\leq$ aplicada de forma independente elemento a elemento de a e b.

$a_{0} \leq b_{0} ; a_{1} \leq b_{1} ; a_{2} \leq b_{2} ; \ldots ; a_{n} \leq b_{n} ;$

ov_lef

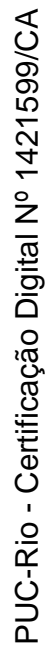

ov_maskf ov_lef(ov_float const a, ov_float const b)

Retorna uma máscara de bits com a operação $\leq$ aplicada de forma independente elemento a elemento de a e b.

$a_{0} \leq b_{0} ; a_{1} \leq b_{1} ; a_{2} \leq b_{2} ; \ldots ; a_{n} \leq b_{n} ;$

ov_loadd

ov_double ov_loadd(double const *addr)

Retorna um vetor SIMD de doubles carregado a partir de um endereço alinhado em memória.

ov_loadf

ov_float ov_loadf(float const $*$ addr)

Retorna um vetor SIMD de floats carregado a partir de um endereço alinhado em memória. 
ov_ltd

ov_maskd ov_ltd(ov_double const a, ov_double const b)

Retorna uma máscara de bits com a operação < aplicada de forma independente elemento a elemento de a e b.

$a_{0}<b_{0} ; a_{1}<b_{1} ; a_{2}<b_{2} ; \ldots ; a_{n}<b_{n}$

ov_ltf

ov_maskf ov_ltf(ov_float const a, ov_float const b)

Retorna uma máscara de bits com a operação < aplicada de forma independente elemento a elemento de a e b.

$a_{0}<b_{0} ; a_{1}<b_{1} ; a_{2}<b_{2} ; \ldots ; a_{n}<b_{n}$

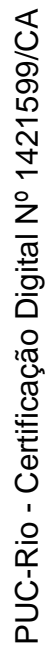

ov_maddd

ov_double ov_maddd(ov_double const a, ov_double const b, ov_double const c)

Retorna um vetor SIMD de doubles com a operação multiply and add aplicada de forma independente elemento a elemento de a, b e c.

$\left(a_{0} * b_{0}\right)+c_{0} ;\left(a_{1} * b_{1}\right)+c_{1} ;\left(a_{2} * b_{2}\right)+c_{2} ; \ldots ;\left(a_{n} * b_{n}\right)+c_{n} ;$

ov_maddf

ov_float ov_maddf(ov_float const a, ov_float const b, ov_float const $c$ )

Retorna um vetor SIMD de floats com a operação multiply and add aplicada de forma independente elemento a elemento de a, b e c.

$\left(a_{0} * b_{0}\right)+c_{0} ;\left(a_{1} * b_{1}\right)+c_{1} ;\left(a_{2} * b_{2}\right)+c_{2} ; \ldots ;\left(a_{n} * b_{n}\right)+c_{n} ;$ 
ov_maxd $C++$

ov_double ov_maxd(double const a, ov_double const b)

Retorna um vetor SIMD de doubles com a operação maior valor aplicada de forma independente elemento a elemento de b com o escalar a.

$\max \left(a, b_{0}\right) ; \max \left(a, b_{1}\right) ; \max \left(a, b_{2}\right) ; \ldots ; \max \left(a, b_{n}\right)$

ov_maxd $\mathrm{d}^{C++}$

ov_double ov_maxd(ov_double const a, double const b)

Retorna um vetor SIMD de doubles com a operação maior valor aplicada de forma independente elemento a elemento de a com o escalar b.

$\max \left(a_{0}, b\right) ; \max \left(a_{1}, b\right) ; \max \left(a_{2}, b\right) ; \ldots ; \max \left(a_{n}, b\right)$

ov_maxd

ov_double ov_maxd(ov_double const a, ov_double const b)

Retorna um vetor SIMD de doubles com a operação maior valor aplicada de forma independente elemento a elemento de a e b.

$\max \left(a_{0}, b_{0}\right) ; \max \left(a_{1}, b_{1}\right) ; \max \left(a_{2}, b_{2}\right) ; \ldots ; \max \left(a_{n}, b_{n}\right)$

ov_maxf ${ }^{C++}$

ov_float ov_maxf(float const a, ov_float const $b$ )

Retorna um vetor SIMD de floats com a operação maior valor aplicada de forma independente elemento a elemento de b com o escalar a.

$\max \left(a, b_{0}\right) ; \max \left(a, b_{1}\right) ; \max \left(a, b_{2}\right) ; \ldots ; \max \left(a, b_{n}\right)$

ov_maxf ${ }^{C++}$

ov_float ov_maxf(ov_float const a, float const b)

Retorna um vetor SIMD de floats com a operação maior valor aplicada de forma independente elemento a elemento de a com o escalar b. 
$\max \left(a_{0}, b\right) ; \max \left(a_{1}, b\right) ; \max \left(a_{2}, b\right) ; \ldots ; \max \left(a_{n}, b\right)$

ov_maxf

ov_float ov_maxf(ov_float const a, ov_float const b)

Retorna um vetor SIMD de floats com a operação maior valor aplicada de forma independente elemento a elemento de a e b.

$\max \left(a_{0}, b_{0}\right) ; \max \left(a_{1}, b_{1}\right) ; \max \left(a_{2}, b_{2}\right) ; \ldots ; \max \left(a_{n}, b_{n}\right)$

ov_mind ${ }^{C++}$

ov_double ov_mind(double const a, ov_double const b)

Retorna um vetor SIMD de doubles com a operação menor valor aplicada de forma independente elemento a elemento de b com o escalar a.

$\min \left(a, b_{0}\right) ; \min \left(a, b_{1}\right) ; \min \left(a, b_{2}\right) ; \ldots ; \min \left(a, b_{n}\right)$

ov_mind ${ }^{C++}$

ov_double ov_mind(ov_double const a, double const b)

Retorna um vetor SIMD de doubles com a operação menor valor aplicada de forma independente elemento a elemento de a com o escalar b.

$\min \left(a_{0}, b\right) ; \min \left(a_{1}, b\right) ; \min \left(a_{2}, b\right) ; \ldots ; \min \left(a_{n}, b\right)$

ov_mind

ov_double ov_mind(ov_double const a, ov_double const b)

Retorna um vetor SIMD de doubles com a operação menor valor aplicada de forma independente elemento a elemento de a e b.

$\min \left(a_{0}, b_{0}\right) ; \min \left(a_{1}, b_{1}\right) ; \min \left(a_{2}, b_{2}\right) ; \ldots ; \min \left(a_{n}, b_{n}\right)$ 
ov_minf ${ }^{C++}$

ov_float ov_minf(float const a, ov_float const $b$ )

Retorna um vetor SIMD de floats com a operação menor valor aplicada de forma independente elemento a elemento de b com o escalar a.

$\min \left(a, b_{0}\right) ; \min \left(a, b_{1}\right) ; \min \left(a, b_{2}\right) ; \ldots ; \min \left(a, b_{n}\right)$

ov_minf ${ }^{C++}$

ov_float ov_minf(ov_float const a, float const b)

Retorna um vetor SIMD de floats com a operação menor valor aplicada de forma independente elemento a elemento de a com o escalar b.

$\min \left(a_{0}, b\right) ; \min \left(a_{1}, b\right) ; \min \left(a_{2}, b\right) ; \ldots ; \min \left(a_{n}, b\right)$

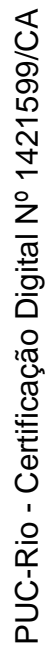

ov_minf

ov_float ov_minf(ov_float const a, ov_float const b)

Retorna um vetor SIMD de floats com a operação menor valor aplicada de forma independente elemento a elemento de a e b.

$\min \left(a_{0}, b_{0}\right) ; \min \left(a_{1}, b_{1}\right) ; \min \left(a_{2}, b_{2}\right) ; \ldots ; \min \left(a_{n}, b_{n}\right)$

ov_msubd

ov_double ov_msubd(ov_double const a, ov_double const b, ov_double const $c$ )

Retorna um vetor SIMD de doubles com a operação multiply and subtract aplicada de forma independente elemento a elemento de a, b e c.

$\left(a_{0} * b_{0}\right)-c_{0} ;\left(a_{1} * b_{1}\right)-c_{1} ;\left(a_{2} * b_{2}\right)-c_{2} ; \ldots ;\left(a_{n} * b_{n}\right)-c_{n} ;$

ov_msubf

ov_float ov_msubf(ov_float const a, ov_float const b, ov_float const c) 
Retorna um vetor SIMD de floats com a operação multiply and subtract aplicada de forma independente elemento a elemento de a, b e c.

$\left(a_{0} * b_{0}\right)-c_{0} ;\left(a_{1} * b_{1}\right)-c_{1} ;\left(a_{2} * b_{2}\right)-c_{2} ; \ldots ;\left(a_{n} * b_{n}\right)-c_{n} ;$

ov_muld

ov_double ov_muld(ov_double const a, ov_double const b)

Retorna um vetor SIMD de doubles com a operação multiplicação aplicada de forma independente elemento a elemento de a e b.

$a_{0} \times b_{0} ; a_{1} \times b_{1} ; a_{2} \times b_{2} ; \ldots ; a_{n} \times b_{n} ;$

ov_mulf

ov_float ov_mulf(ov_float const a, ov_float const b)

Retorna um vetor SIMD de floats com a operação multiplicação aplicada de forma independente elemento a elemento de a e b.

$a_{0} \times b_{0} ; a_{1} \times b_{1} ; a_{2} \times b_{2} ; \ldots ; a_{n} \times b_{n} ;$

ov_ned

ov_maskd ov_ned(ov_double const a, ov_double const b)

Retorna uma máscara de bits com a operação != aplicada de forma independente elemento a elemento de a e b.

$a_{0} \neq b_{0} ; a_{1} \neq b_{1} ; a_{2} \neq b_{2} ; \ldots ; a_{n} \neq b_{n}$

ov_nef

ov_maskf ov_nef(ov_float const a, ov_float const b)

Retorna uma máscara de bits com a operação != aplicada de forma independente elemento a elemento de a e b.

$a_{0} \neq b_{0} ; a_{1} \neq b_{1} ; a_{2} \neq b_{2} ; \ldots ; a_{n} \neq b_{n}$ 
ov_not_alignedd

unsigned long ov_not_alignedd(double const $*$ addr)

Retorna zero se addr está alinhado, caso contrário retorna o número de elementos (double) adicionais ao maior endereço alinhado que é menor ou igual à addr. Por exemplo, em uma máquina SIMD, se o array double *x está alinhado o retorno para addr=\&x[1] é 1, e o retorno para addr $=\& x$ [OV_DOUBLE_WIDTH] é zero.

Em uma CPU escalar esta função sempre retorna 0.

Também fornecemos a função ov_is_alignedd, que implementamos da seguinte forma:

\#define ov_is_alignedd(addr) (!ov_not_alignedd(addr))

ov_not_alignedf

unsigned long ov_not_alignedf(float const *addr)

Retorna zero se addr está alinhado, caso contrário retorna o número de elementos (float) adicionais ao maior endereço alinhado que é menor ou igual à addr. Por exemplo, em uma máquina SIMD, se o array float $* x$ está alinhado o retorno para $\operatorname{addr}=\& x[1]$ é 1 , e o retorno para addr $=\& x$ [OV_FLOAT_WIDTH] é zero.

Em uma CPU escalar esta função sempre retorna 0.

Também fornecemos a função ov_is_alignedf, que implementamos da seguinte forma:

\#define ov_is_alignedf(addr) (!ov_not_alignedf(addr))

ov_rcpd

ov_double ov_rcpd(ov_double const a)

Retorna um vetor SIMD de doubles com a operação reciprocal aplicada de forma independente em todos os elementos de a.

$\frac{1}{a_{0}} ; \frac{1}{a_{1}} ; \frac{1}{a_{2}} ; \ldots ; \frac{1}{a_{n}} ;$ 
ov_rcpf

ov_float ov_rcpf(ov_float const a)

Retorna um vetor SIMD de floats com a operação reciprocal aplicada de forma independente em todos os elementos de a.

$\frac{1}{a_{0}} ; \frac{1}{a_{1}} ; \frac{1}{a_{2}} ; \ldots ; \frac{1}{a_{n}}$

ov_rsqrtd

ov_double ov_rsqrtd(ov_double const a)

Retorna um vetor SIMD de doubles com a operação raiz quadrada inversa aplicada de forma independente em todos os elementos de a.

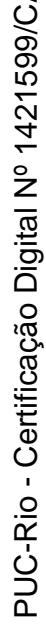

$\frac{1}{\sqrt{a_{0}}} ; \frac{1}{\sqrt{a_{1}}} ; \frac{1}{\sqrt{a_{2}}} ; \ldots ; \frac{1}{\sqrt{a_{n}}}$

ov_rsqrtf

ov_float ov_rsqrtf(ov_float const a)

Retorna um vetor SIMD de floats com a operação raiz quadrada inversa aplicada de forma independente em todos os elementos de a.

$\frac{1}{\sqrt{a_{0}}} ; \frac{1}{\sqrt{a_{1}}} ; \frac{1}{\sqrt{a_{2}}} ; \ldots ; \frac{1}{\sqrt{a_{n}}} ;$

ov_setd

ov_double ov_setd(double const a)

Retorna um vetor SIMD de doubles com o escalar $a$ repetido em todas as posições do vetor.

$a ; a ; a ; \ldots ; a ;$

ov_setf

ov_float ov_setf(float const a) 
Retorna um vetor SIMD de floats com o escalar a repetido em todas as posições do vetor.

$a ; a ; a ; \ldots ; a ;$

ov_sqrtd

ov_double ov_sqrtd(ov_double const a)

Retorna um vetor SIMD de doubles com a operação raiz quadrada aplicada de forma independente em todos os elementos de a.

$\sqrt{a_{0}} ; \sqrt{a_{1}} ; \sqrt{a_{2}} ; \ldots ; \sqrt{a_{n}}$

ov_sqrtf

ov_float ov_sqrtf(ov_float const a)

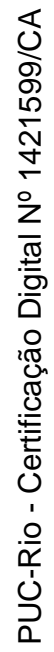

Retorna um vetor SIMD de floats com a operação raiz quadrada aplicada de forma independente em todos os elementos de a.

$\sqrt{a_{0}} ; \sqrt{a_{1}} ; \sqrt{a_{2}} ; \ldots ; \sqrt{a_{n}}$

ov_std

void ov_std(double *addr, ov_double const a)

Armazena um vetor SIMD de doubles em um endereço alinhado em memória.

ov_stf

void ov_stf(float *addr, ov_float const a)

Armazena um vetor SIMD de floats em um endereço alinhado em memória.

ov_stream_std

void ov_stream_std(double $*$ addr, ov_double const a) 
Armazena um vetor SIMD de doubles em um endereço alinhado em memória.

Em algumas CPUs esta instrução move uma linha de cache completa para a memória, e pode trazer mais desempenho evitando um load/update/store.

Em outras arquiteturas, que não possuem este tipo de store, esta função se comporta igual a função ov_std.

ov_stream_stf

void ov_stream_stf(float *addr, ov_float const a)

Armazena um vetor SIMD de floats em um endereço alinhado em memória.

Em algumas CPUs esta instrução move uma linha de cache completa para a memória, e pode trazer mais desempenho evitando um load/update/store.

Em outras arquiteturas, que não possuem este tipo de store, esta função se comporta igual a função ov_stf.

ov_subd

ov_double ov_subd(ov_double const a, ov_double const b)

Retorna um vetor SIMD de doubles com a operação subtração aplicada de forma independente elemento a elemento de a e b.

$a_{0}-b_{0} ; a_{1}-b_{1} ; a_{2}-b_{2} ; \ldots ; a_{n}-b_{n} ;$

ov_subf

ov_float ov_subf(ov_float const a, ov_float const b)

Retorna um vetor SIMD de floats com a operação subtração aplicada de forma independente elemento a elemento de a e b.

$a_{0}-b_{0} ; a_{1}-b_{1} ; a_{2}-b_{2} ; \ldots ; a_{n}-b_{n} ;$

ov_uldd

ov_double ov_uldd(double const *addr)

Retorna um vetor SIMD de doubles carregado a partir de um endereço desalinhado em memória. 
ov_uldf

ov_float ov_uldf(float const $*$ addr)

Retorna um vetor SIMD de floats carregado a partir de um endereço desalinhado em memória.

$\operatorname{sqrtd}^{C++}$

ov_double sqrtd(ov_double const a)

Retorna um vetor SIMD de doubles com a operação raiz quadrada aplicada de forma independente em todos os elementos de a.

$\sqrt{a_{0}} ; \sqrt{a_{1}} ; \sqrt{a_{2}} ; \ldots ; \sqrt{a_{n}}$

$\operatorname{sqrtf}^{C++}$

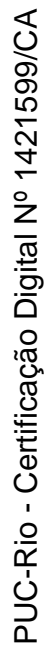

ov_float sqrtf(ov_float const a)

Retorna um vetor SIMD de floats com a operação raiz quadrada aplicada de forma independente em todos os elementos de a.

$\sqrt{a_{0}} ; \sqrt{a_{1}} ; \sqrt{a_{2}} ; \ldots ; \sqrt{a_{n}}$ 
B

\section{Documentação HLIB}

Para utilizar a HLIB basta fazer um USE do módulo Fortran 90 da HLIB, conforme o código a seguir:

\section{USE HLIB}

Como todas as sub-rotinas estão definidas no módulo HLIB, o compilador Fortran 90 será capaz de detectar se as invocações respeitam a interface durante a compilação.

\section{B.1}

\section{Sub-rotinas}

Todas as sub-rotinas da HLIB retornam um código de erro. Definimos o valor zero para sucesso e um valor diferente de zero para erro.

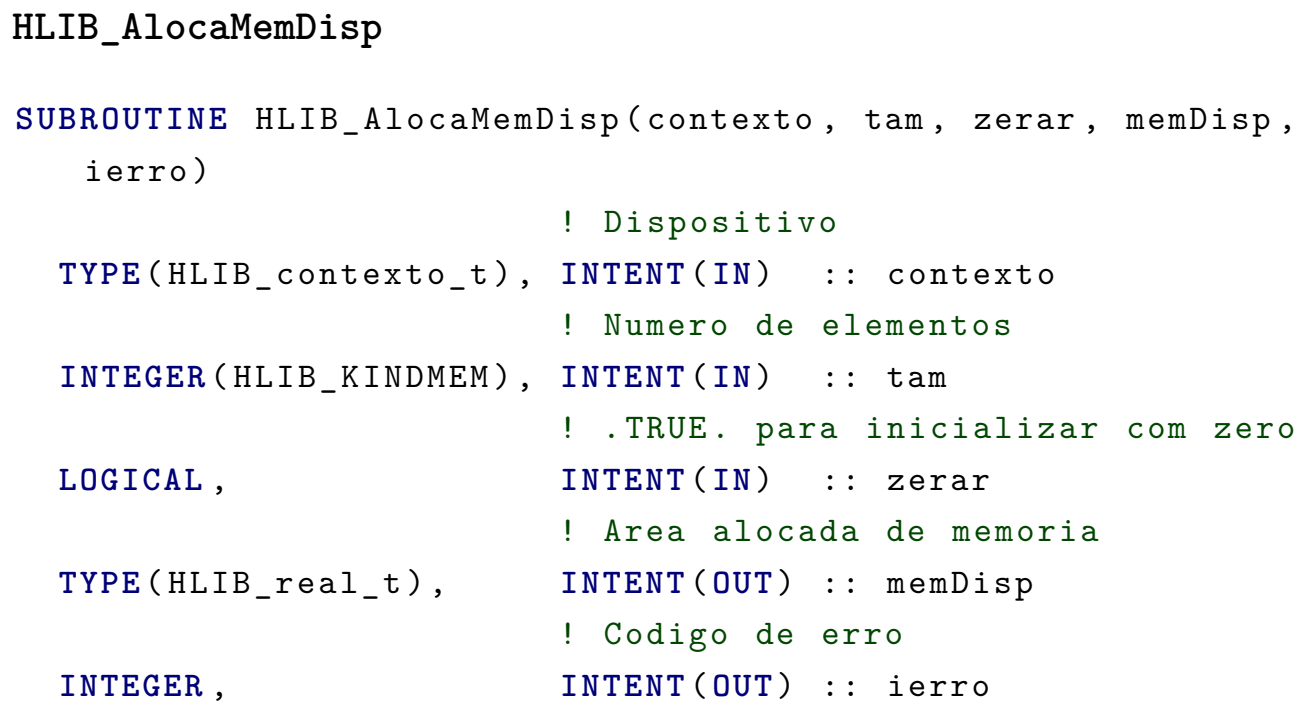

Aloca uma memória com tamanho tam bytes no dispositivo associado ao contexto.

Se o argumento zerar é . TRUE. a memória é inicializada com zero.

Suportamos interfaces para os seguintes tipos do argumento memDisp: HLIB_real_t, HLIB_double_t, HLIB_int_t e HLIB_long_t. 


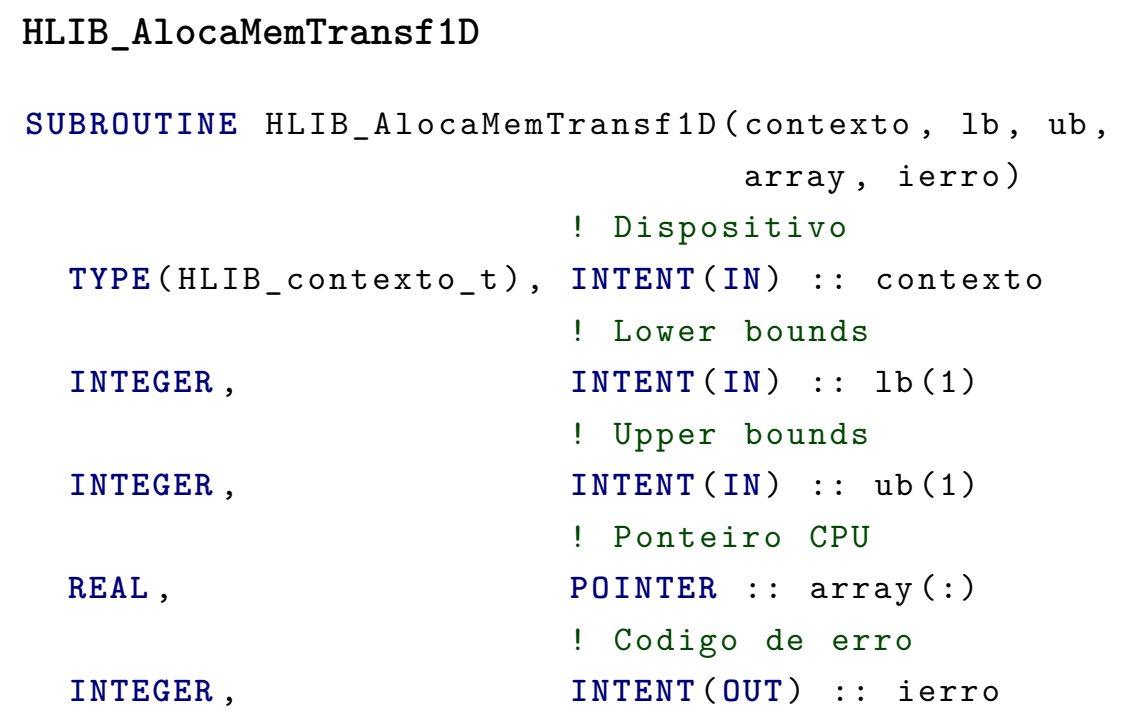

Aloca uma memória da CPU otimizada para ser utilizada em transferências do tipo $\mathrm{CPU} \Leftrightarrow$ dispositivo. Em alguns backends esta memória não pode ser paginada (pinned), com isso, nestes backends, temos um ganho de desempenho nas transferências $\mathrm{CPU} \Leftrightarrow$ dispositivo.

O lower bound do ponteiro é definido pelo argumento $1 \mathrm{~b}(1)$ e o upper bound é definido pelo argumento ub(1). Ou seja, a área é alocada com as seguintes dimensões $\operatorname{array}(\mathrm{lb}(1): \mathrm{ub}(1))$.

O desenvolvedor deve somente desalocar esta memória com a sub-rotina HLIB_DesalocaMemTransf.

Suportamos interfaces para os seguintes tipos do argumento array: REAL, DOUBLE PRECISION, INTEGER (KIND=HLIB_I32) e INTEGER (KIND=HLIB_I64).

\section{HLIB_AlocaMemTransf2D}

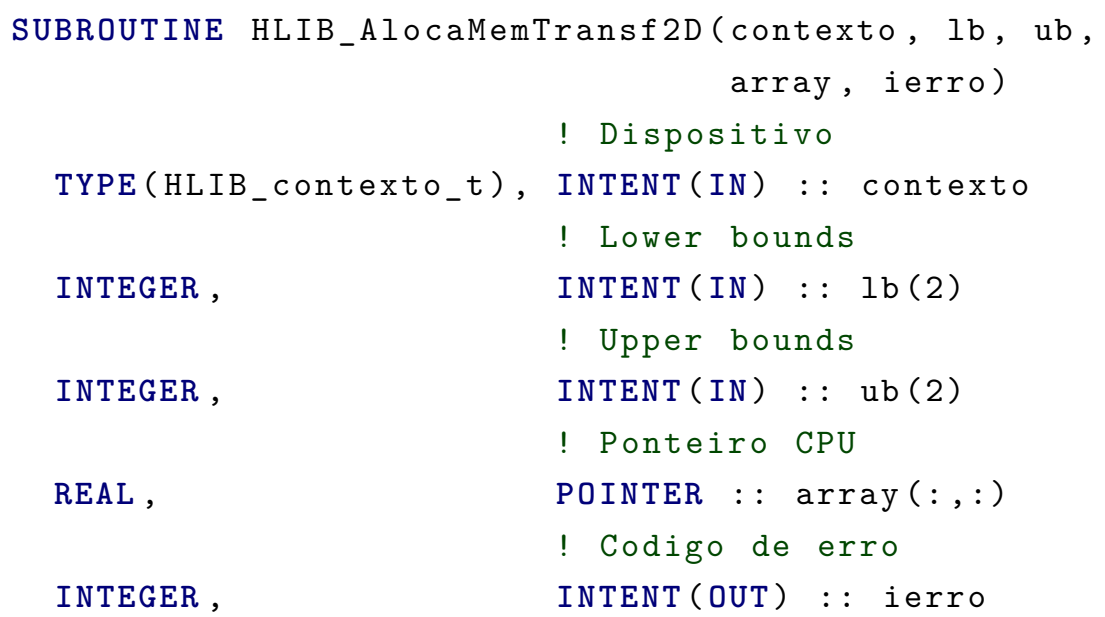

Aloca uma memória da CPU otimizada para ser utilizada em transferências do tipo $\mathrm{CPU} \Leftrightarrow$ dispositivo. Em alguns backends esta memória não 
pode ser paginada (pinned), com isso, nestes backends, temos um ganho de desempenho nas transferências $\mathrm{CPU} \Leftrightarrow$ dispositivo.

O lower bound do ponteiro é definido pelo argumento $1 \mathrm{~b}(1: 2)$ e o upper bound é definido pelo argumento ub(1:2). Ou seja, a área é alocada com as seguintes dimensões $\operatorname{array}(1 b(1): u b(1), l b(2): u b(2))$.

O desenvolvedor deve somente desalocar esta memória com a sub-rotina HLIB_DesalocaMemTransf.

Suportamos interfaces para os seguintes tipos do argumento array: REAL, DOUBLE PRECISION, INTEGER(KIND=HLIB_I32) e INTEGER (KIND=HLIB_I64).

\section{HLIB_AlocaMemTransf 3D}

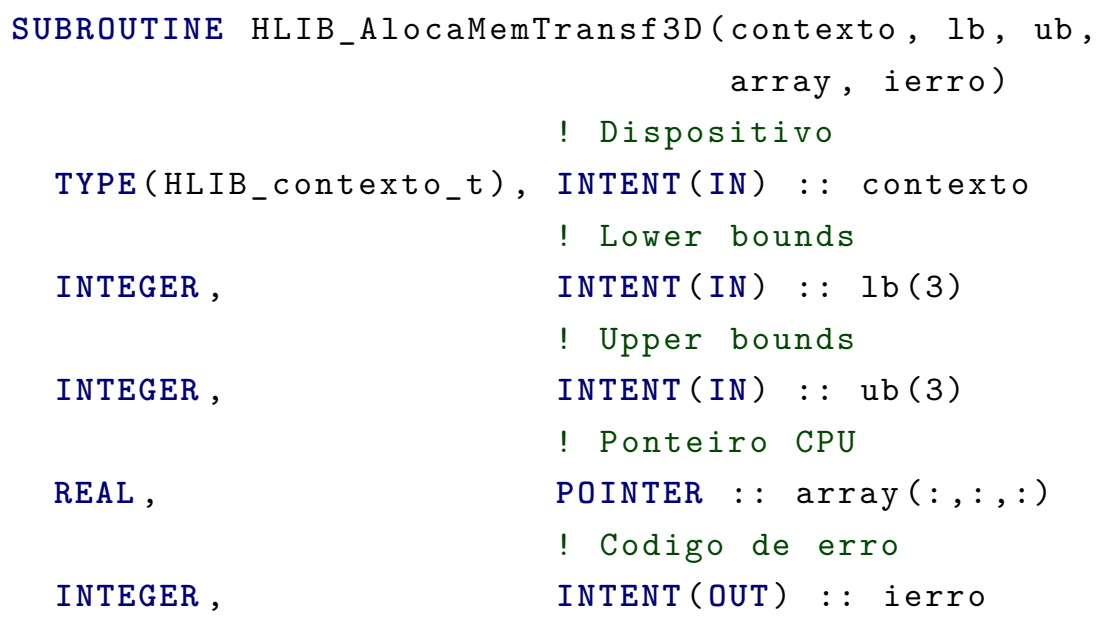

Aloca uma memória da CPU otimizada para ser utilizada em transferências do tipo $\mathrm{CPU} \Leftrightarrow$ dispositivo. Em alguns backends esta memória não pode ser paginada (pinned), com isso, nestes backends, temos um ganho de desempenho nas transferências $\mathrm{CPU} \Leftrightarrow$ dispositivo.

O lower bound do ponteiro é definido pelo argumento $1 \mathrm{~b}(1: 3)$ e o upper bound é definido pelo argumento ub(1:3). Ou seja, a área é alocada com as seguintes dimensões array (lb(1): ub(1), $1 \mathrm{~b}(2): \mathrm{ub}(2), 1 \mathrm{~b}(3): \mathrm{ub}(3))$.

O desenvolvedor deve somente desalocar esta memória com a sub-rotina HLIB_DesalocaMemTransf.

Suportamos interfaces para os seguintes tipos do argumento array: REAL, DOUBLE PRECISION, INTEGER(KIND=HLIB_I32) e INTEGER (KIND=HLIB_I64).

\section{HLIB_CopiaDispCPU}

SUBROUTINE HLIB_CopiaDispCPU(contexto, memDisp, offset, arrayCPU, dir, ierro) 


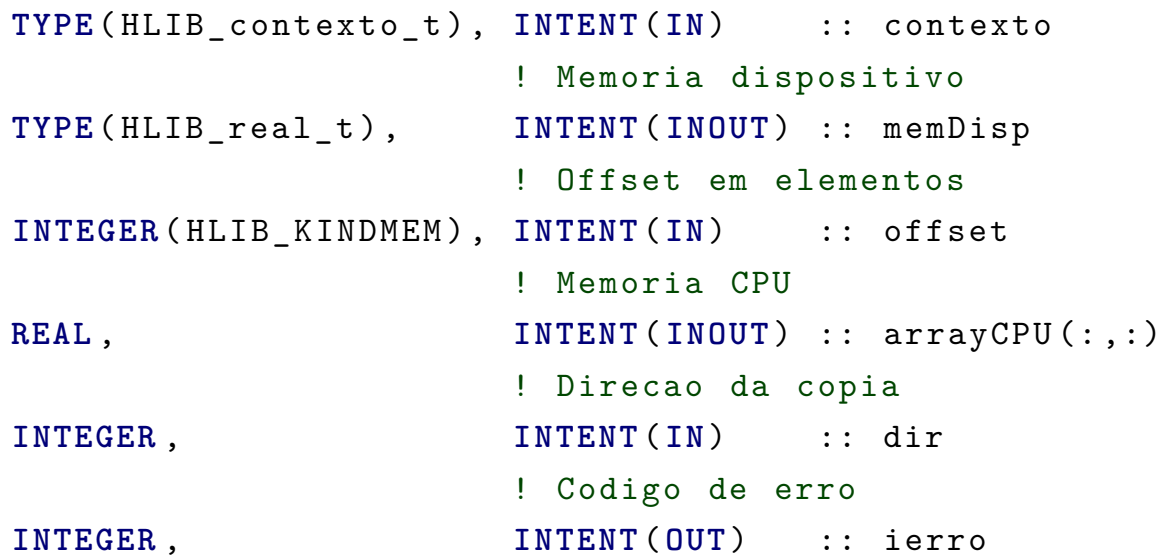

Faz a cópia entre uma memória alocada no dispositivo associado ao contexto e uma memória alocada na CPU. A memória da CPU deve ser alocada previamente com uma das rotinas HLIB_AlocaMemTransf*.

O desenvolvedor deve informar o offset (em elementos) para a memória do dispositivo, que é mapeada pelo argumento memDisp.

O tamanho da cópia é determinado pelos lower e upper bounds de arrayCPU. O desenvolvedor deve passar lower e upper bounds que definam uma área contígua de memória.

O argumento dir define a direção da cópia, que pode ser HLIB_DESTINO_CPU Ou HLIB_ORIGEM_CPU.

Como armazenamos o tamanho da área alocada no dispositivo, antes de efetuar a cópia verificamos se ocorrerá uma invasão de memória no dispositivo.

Suportamos interfaces para os seguintes pares de argumentos arrayCPU e memDisp conforme a tabela a seguir.

\begin{tabular}{|l|l|}
\hline arrayCPU & memDisp \\
\hline REAL, DIMENSION $(:)$ & HLIB_real_t \\
\hline REAL, DIMENSION $(:,:)$ & HLIB_real_t \\
\hline REAL, DIMENSION $(:,:,:)$ & HLIB_real_t \\
\hline DOUBLE PRECISION, DIMENSION $(:)$ & HLIB_double_t \\
\hline DOUBLE PRECISION, DIMENSION $(:,:)$ & HLIB_double_t \\
\hline DOUBLE PRECISION, DIMENSION $(:,:,:)$ & HLIB_double_t \\
\hline INTEGER (KIND=HLIB_I32), DIMENSION $(:)$ & HLIB_int_t \\
\hline INTEGER (KIND=HLIB_I32), DIMENSION $(:,:)$ & HLIB_int_t \\
\hline INTEGER (KIND=HLIB_I32), DIMENSION $(:,:,:)$ & HLIB_int_t \\
\hline INTEGER (KIND=HLIB_I64), DIMENSION $(:)$ & HLIB_long_t \\
\hline INTEGER (KIND=HLIB_I64), DIMENSION $(:,:)$ & HLIB_long_t \\
\hline INTEGER (KIND=HLIB_I64), DIMENSION $(:,:,:)$ & HLIB_long_t \\
\hline
\end{tabular}




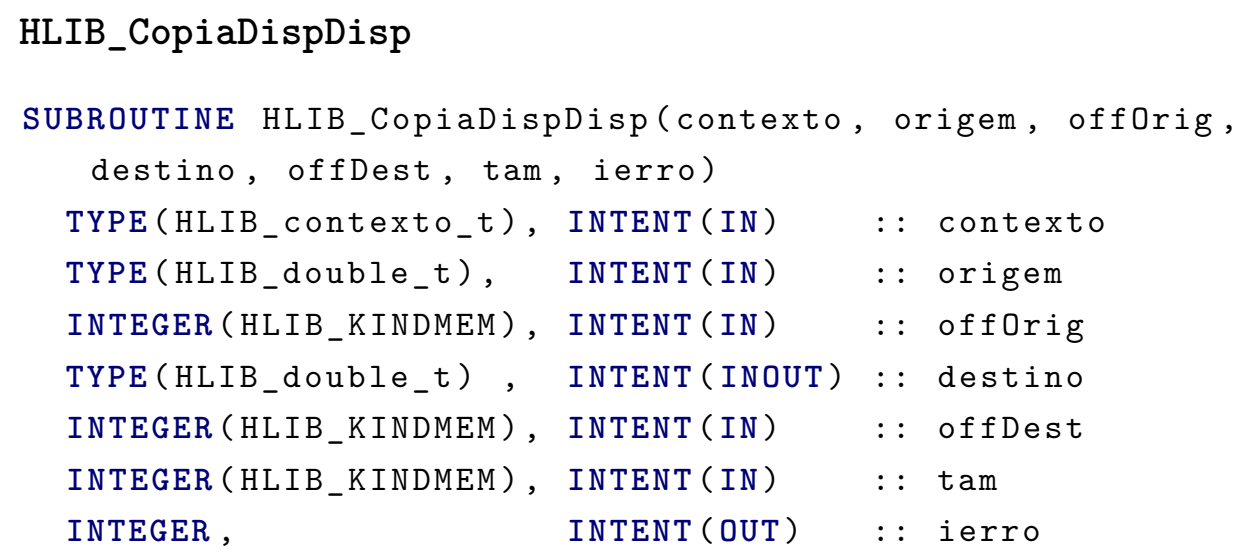

Faz a cópia entre memórias alocadas no dispositivo associado ao contexto.

O desenvolvedor deve informar os offsets (em elementos) para a origem e destino. $\mathrm{O}$ argumento tam define a quantidade de elementos a serem copiados.

Como armazenamos o tamanho da área alocada no dispositivo, antes de efetuar a cópia verificamos se ocorrerá uma invasão de memória.

Suportamos interfaces para os seguintes tipos dos argumentos origem e destino: HLIB_real_t, HLIB_double_t, HLIB_int_t e HLIB_long_t. O argumento origem deve ser do mesmo tipo do argumento destino.

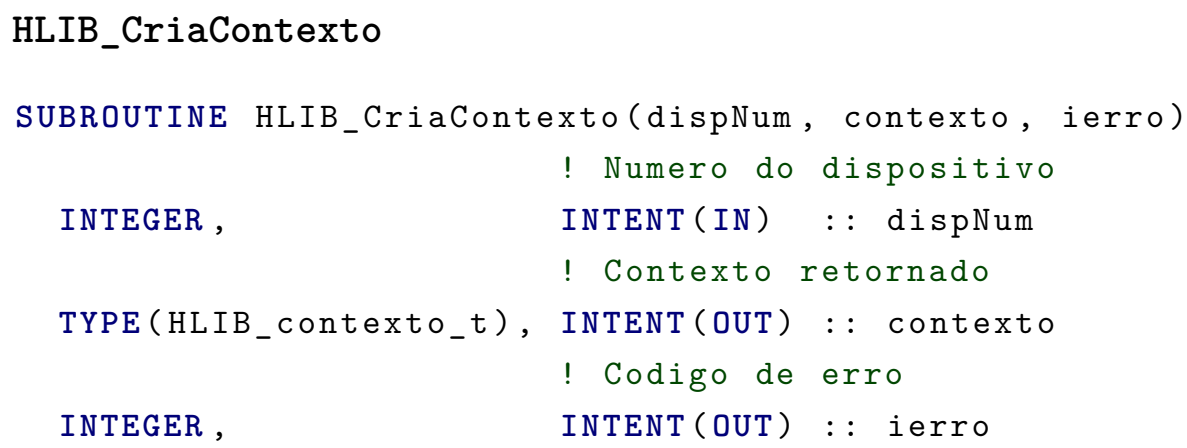

Cria um contexto com o dispositivo dispNum mod ndisp. Onde ndisp é o total de dispositivos. O desenvolvedor deve informar no argumento dispNum o número do dispositivo a ser utilizado.

O processo de inicialização compreende em verificar quantos dispositivos (ndisp) existem na máquina e associar ao contexto o dispositivo dispNun mod ndisp, onde dispNum é o número do dispositivo passado para a sub-rotina HLIB_CriaContexto.

Em alguns backends como o OpenCL e hStreams, também é necessário criar um fila de execução para as operações internas da biblioteca. Em CUDA não é necessário a criação desta fila, pois existem chamadas síncronas que não necessitam de uma fila, um objeto cudaStream neste caso. 


\section{HLIB_CriaFila}

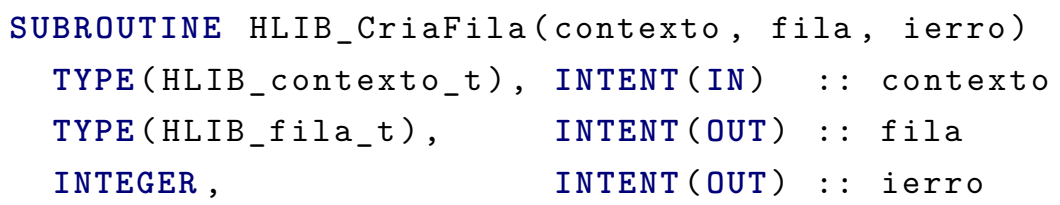

Cria uma fila de execução para enfileirar comandos para execução no dispositivo associado ao contexto.

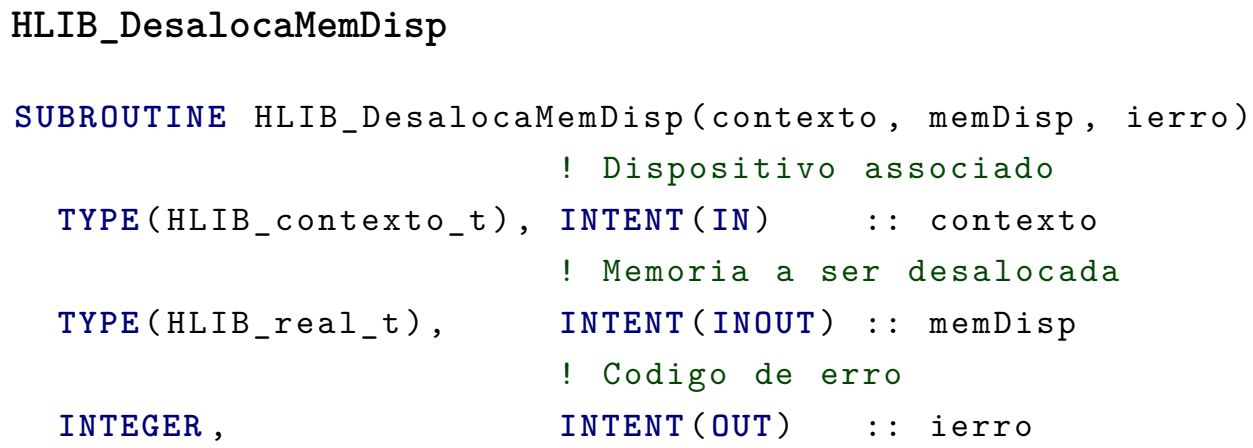

Desaloca uma memória previamente alocada com a sub-rotina HLIB_AlocaMemDisp no dispositivo associado ao contexto.

Suportamos interfaces para os seguintes tipos do argumento memDisp: HLIB_real_t, HLIB_double_t, HLIB_int_t e HLIB_long_t.

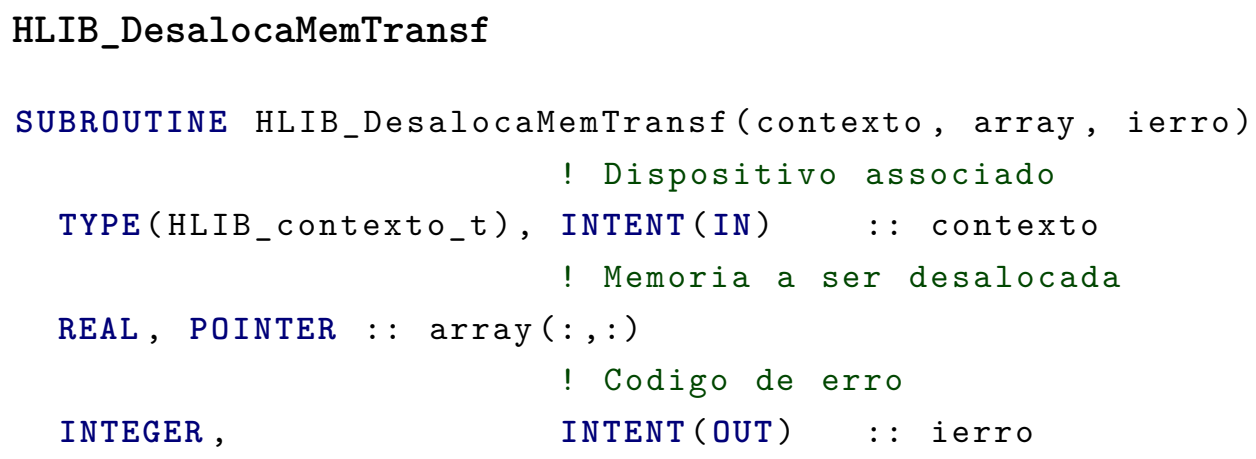

Desaloca uma memória previamente alocada com uma das sub-rotinas HLIB_AlocaMemTransf* no dispositivo associado ao contexto.

Suportamos interfaces para os seguintes tipos do argumento array: REAL, DOUBLE PRECISION, INTEGER(KIND=HLIB_I32) e INTEGER(KIND=HLIB_I64). 


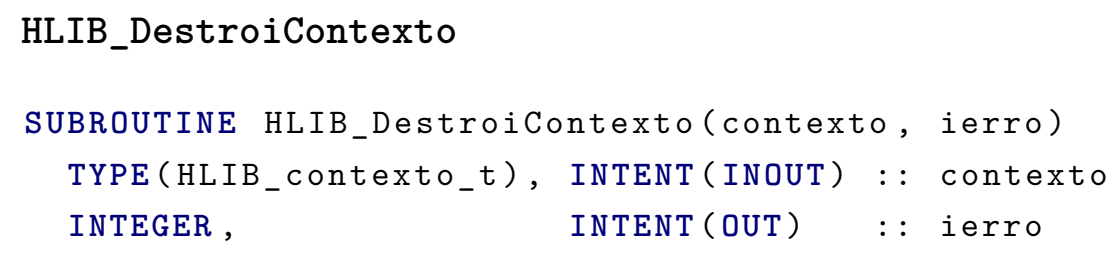

Destrói o contexto com o dispositivo. Após a destruição o objeto contexto não pode ser utilizado por outras sub-rotinas.

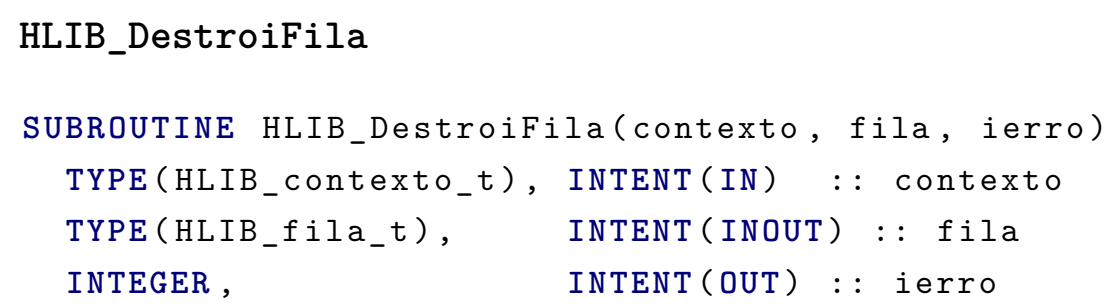

Destrói a fila no dispositivo associado ao contexto.

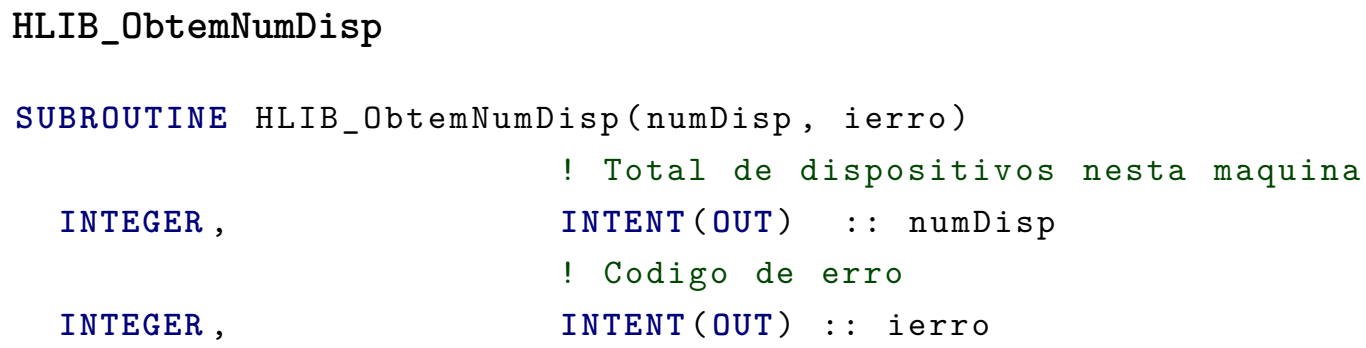

Retorna no argumento numDisp o número de dispositivos disponíveis na máquina.

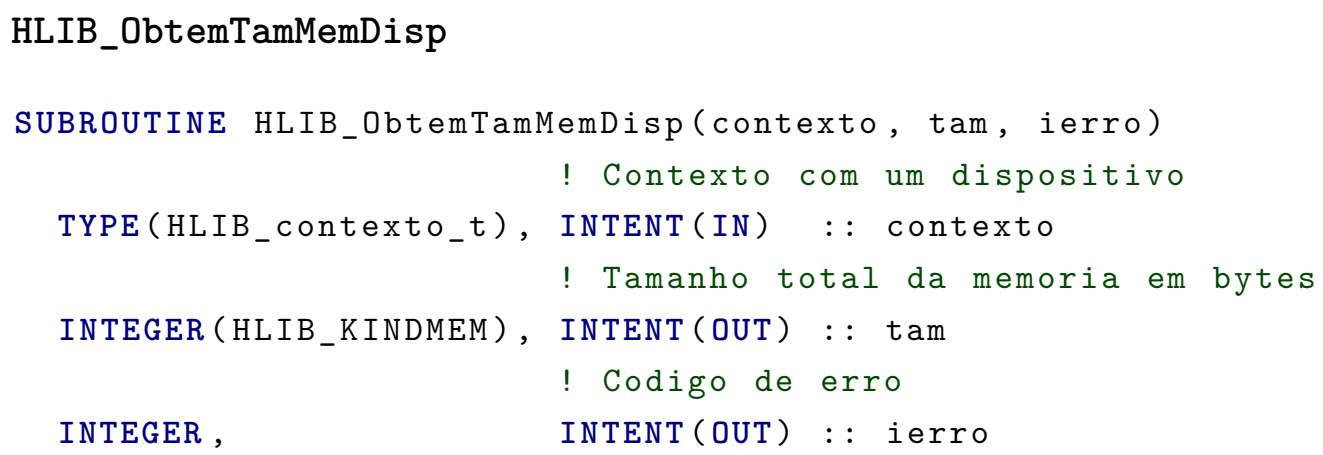

Retorna no argumento tam o total de bytes da memória do dispositivo associado ao contexto. 


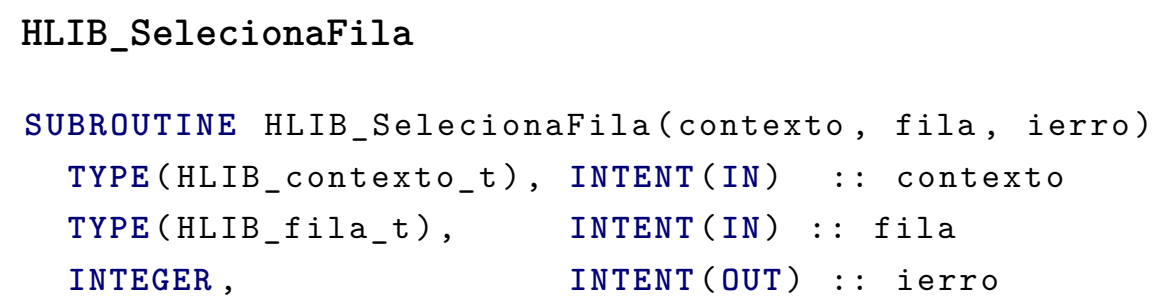

Seleciona o objeto fila como sendo a fila a ser utilizada pelas sub-rotinas da HLIB. Todas chamadas HLIB subsequentes serão enfileiradas nessa fila.

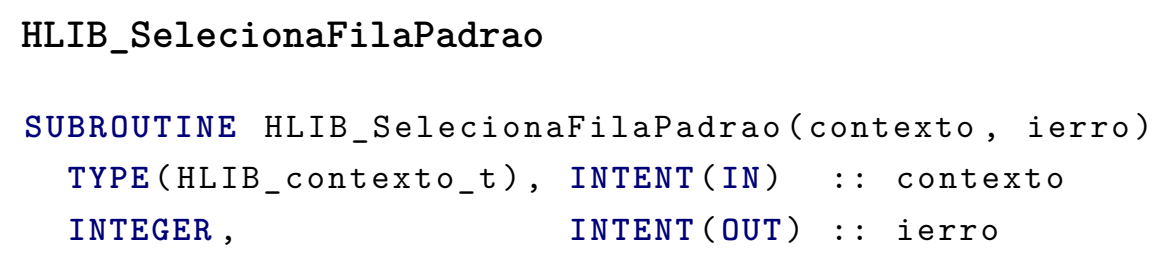

Seleciona a fila síncrona padrão, com isso, todas as invocações de subrotinas da HLIB neste contexto passam a bloquear em relação a CPU.

Esse é o comportamento padrão inicial da HLIB.

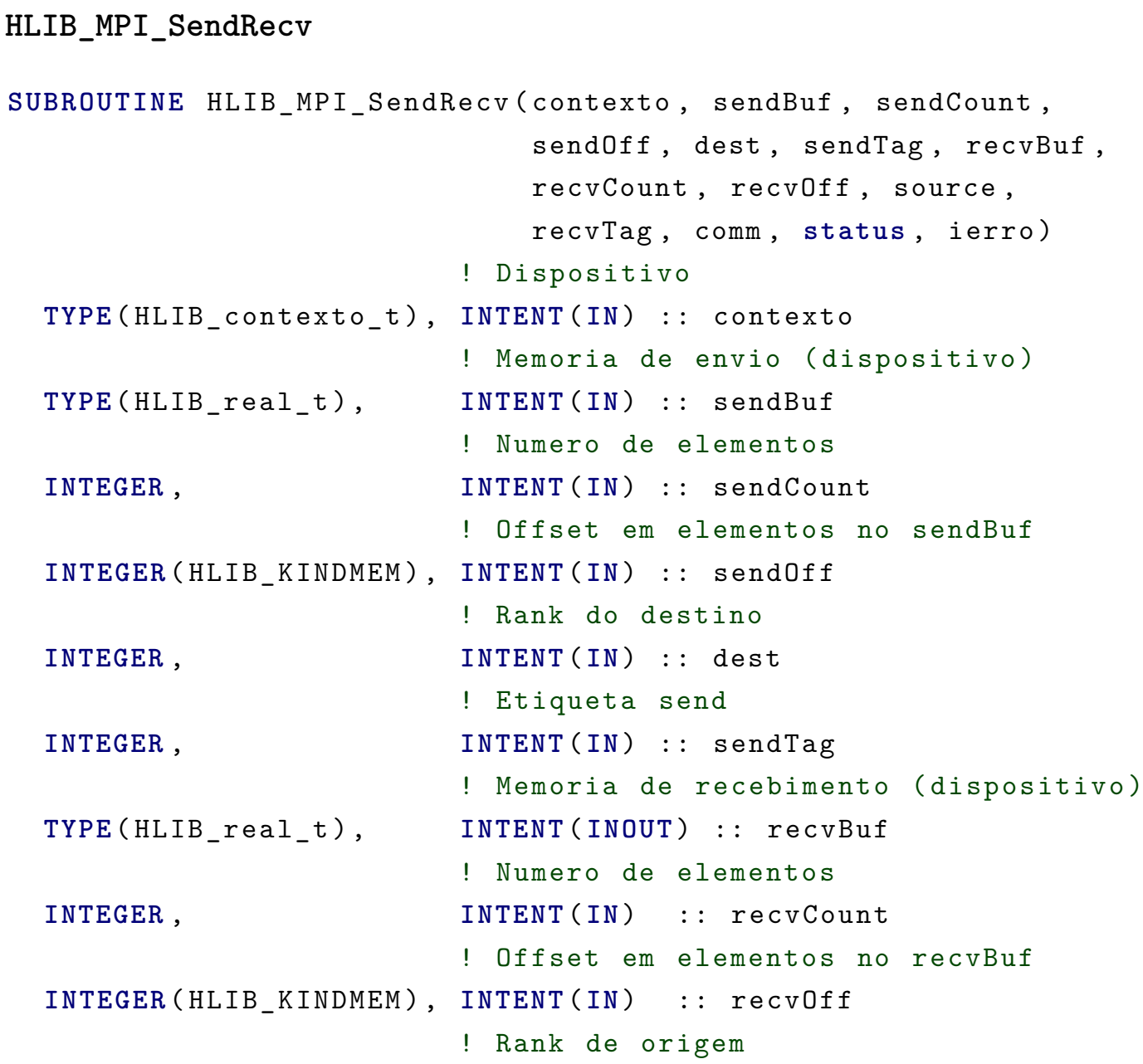




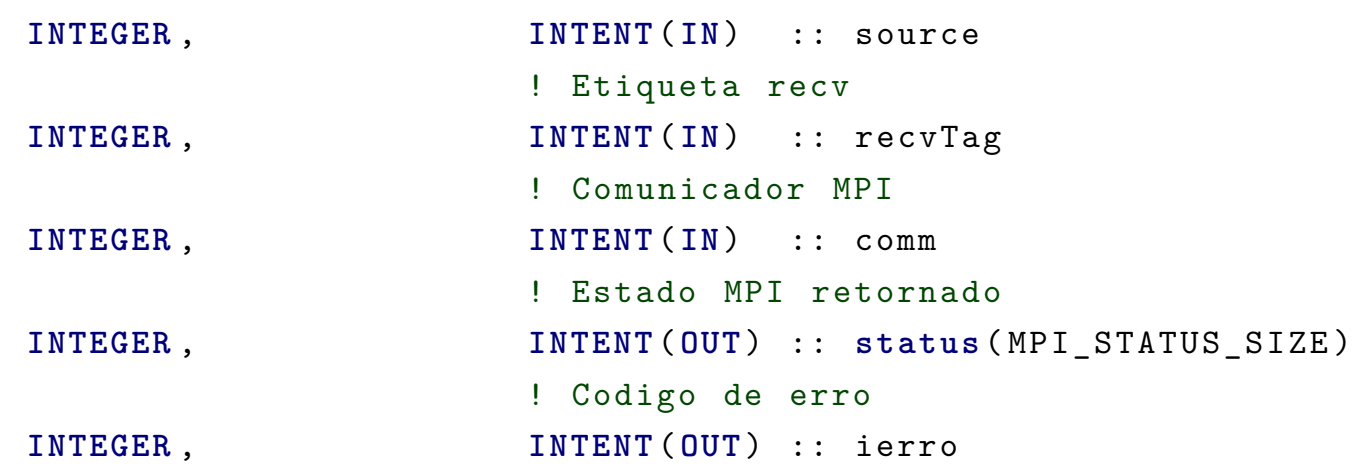

Faz um MPI_SendRecv utilizando buffers alocados no dispositivo associado ao contexto.

\section{HLIB_Sincroniza}

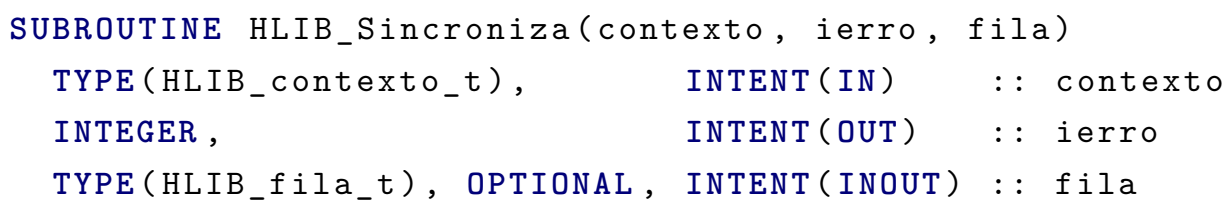

Se o argumento opcional fila estiver presente, esta função aguarda o término de todos os comandos enfileirados no objeto fila. Senão aguarda o término de todos os comandos deste processo enfileirados no dispositivo associado ao contexto.

Esta sub-rotina bloqueia a execução na CPU até o término dos comandos.

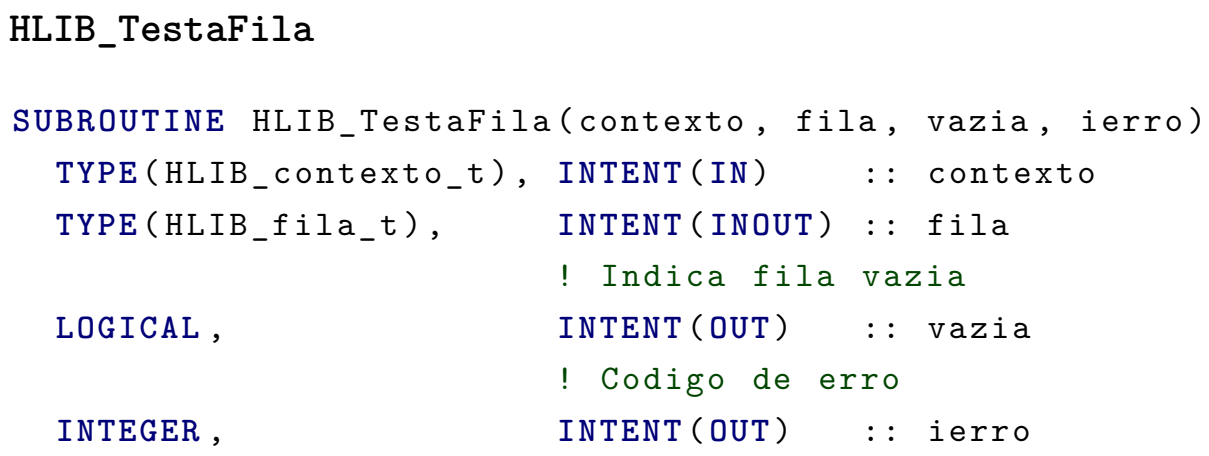

Testa se todos os comandos da fila terminaram, ou seja, testa se a fila está vazia. Esta rotina retorna o estado da fila no argumento vazia.

Esta sub-rotina não bloqueia a execução até o término dos comandos, ela apenas retorna o estado da fila. 


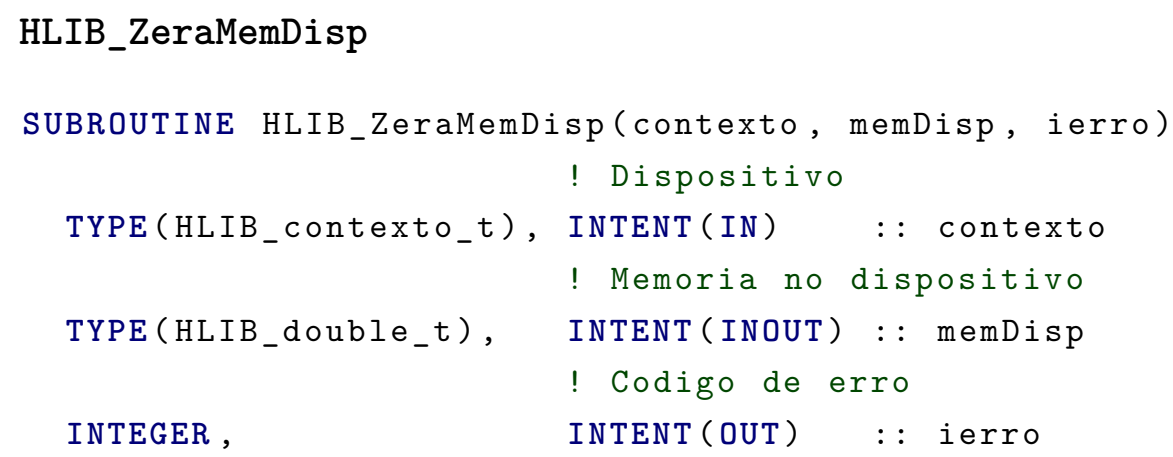

Preenche com zero (0x00) todos os bytes da memória memDisp alocada no dispositivo associado ao contexto.

Suportamos interfaces para os seguintes tipos do argumento memDisp: HLIB_real_t, HLIB_double_t, HLIB_int_t e HLIB_long_t. 